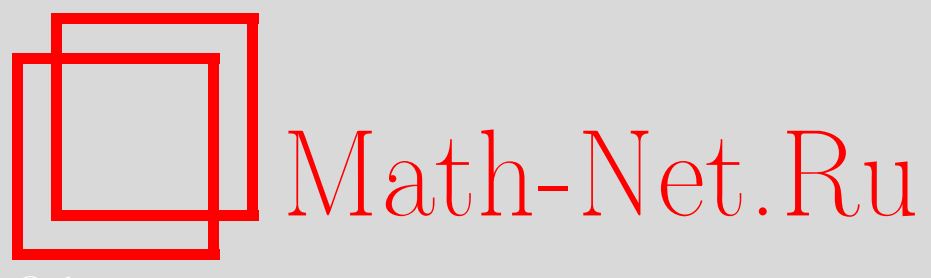

С. П. Суетин, Распределение нулей полиномов Паде и аналитическое продолжение, УМH, 2015, том 70, выпуск $5,121-174$

DOI: https://doi.org/10.4213/rm9675

Использование Общероссийского математического портала Math-Net.Ru подразумевает, что вы прочитали и согласны с пользовательским соглашением http://www . mathnet.ru/rus/agreement

Параметры загрузки:

IP : 44.207 .124 .84

26 апреля 2023 г., 16:10:37

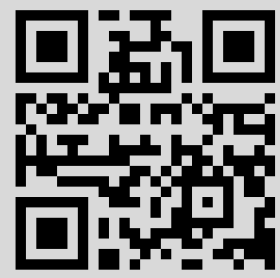




\section{Распределение нулей полиномов Паде и аналитическое продолжение}

\section{С. П. Суетин}

Обсуждается задача аналитического продолжения многозначной аналитической функции с конечным множеством точек ветвления на римановой сфере. Основное внимание уделяется аппроксимациям Паде - классическим (одноточечным), многоточечным аппроксимациям Паде и аппроксимациям Эрмита-Паде.

Основной результат работы - теорема о распределении нулей и сходимости аппроксимаций Эрмита-Паде для набора $\left[1, f, f^{2}\right]$, где многозначная функция $f$ принадлежит так называемому классу Лагерра $\mathscr{L}$.

Библиография: 128 названий.

Ключевые слова: аналитическое продолжение, непрерывные дроби, ортогональные многочлены, рациональные аппроксимации, полиномы Паде, полиномы Эрмита-Паде, распределение нулей, GRS-метод, сходимость по емкости.

DOI: $10.4213 / \mathrm{rm} 9675$

\section{СОДЕРЖАНИЕ}

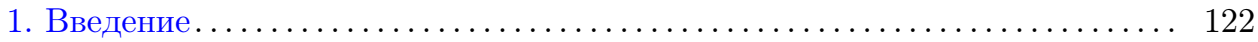

2. Наилучшие рациональные аппроксимации ................... 125

2.1. Теория Колчина и функциональные аналоги теоремы Туэ-Зигеля-Рота........................................ 125

2.2. Аппроксимации Паде: теория Шталя ...................... 128

2.3. Многоточечные аппроксимации Паде: теория Буслаева ........ 137

2.4. Аппроксимации Эрмита-Паде ......................... 139

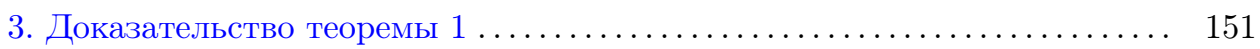

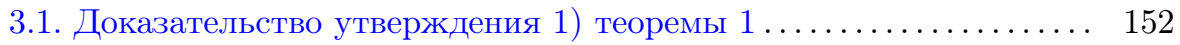

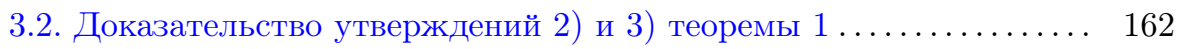

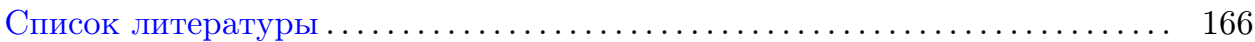

Исследование выполнено за счет гранта Российского научного фонда (проект № 14-50-00005).

(C) С. П. Суетин, 2015 


\section{1. Введение}

Задача аналитического продолжения - классическая задача комплексного анализа. Существуют разнообразные подходы к ее решению: см. прежде всего [18], а также [15], [19], [69] и [123]. В настоящей работе обсуждается один из классических методов решения задачи аналитического продолжения, а именно метод, основанный на конструкции аппроксимаций Паде. Говоря об аппроксимациях Паде (далее АП), мы в настоящей работе следуем терминологии, используемой в работах [27]-[30], в которых под аппроксимациями Паде понимаются классические (одноточечные) АП, многоточечные АП и аппроксимации Эрмита-Паде. Аналогичной терминологии мы придерживаемся, говоря о полиномах Паде: под полиномами Паде понимаются как собственно полиномы Паде, так и полиномы, соответствующие многоточечным АП и аппроксимациям Әрмита-Паде.

Пусть функция $f$ голоморфна в бесконечно удаленной точке $z=\infty: f \in$ $\mathscr{H}(\infty)$. Всюду в дальнейшем предполагается, что $f$-многозначная аналитическая функция на римановой сфере $\overline{\mathbb{C}}$ с конечным множеством особых точек $\Sigma \subset \mathbb{C}, \operatorname{Card} \Sigma<\infty$, причем хотя бы одна из точек этого множества $\Sigma$ является точкой ветвления функции $f$. Тем самым, $f$ - многозначная аналитическая функция в области $\overline{\mathbb{C}} \backslash \Sigma$. При фиксированном множестве $\Sigma$ класс всех таких функций $f$ мы будем обозначать через $\mathscr{A}^{0}(\overline{\mathbb{C}} \backslash \Sigma)$ :

$$
\mathscr{A}^{0}(\overline{\mathbb{C}} \backslash \Sigma):=\mathscr{A}(\overline{\mathbb{C}} \backslash \Sigma) \backslash \mathscr{H}(\overline{\mathbb{C}} \backslash \Sigma)
$$

(подробнее см. ниже п. 2.1).

Пусть аналитическая функция $f$ задана явным представлением

$$
f(z)=\prod_{j=1}^{p}\left(z-a_{j}\right)^{\alpha_{j}}, \quad \alpha_{j} \in \mathbb{C} \backslash \mathbb{Z}, \quad \sum_{j=1}^{p} \alpha_{j}=0
$$

где все точки $a_{j} \in \mathbb{C}$ попарно различны: $a_{j} \neq a_{k}$ при $j \neq k$; в дальнейшем, в зависимости от рассматриваемых конкретных задач, мы налагаем в представлении (1) на точки ветвления $a_{j}$ и параметры $\alpha_{j}$ те или иные дополнительные условия; см., например, (41). Функция $f$ - многозначная аналитическая функция в расширенной комплексной плоскости $\overline{\mathbb{C}}$ с конечным множеством точек ветвления $\Sigma=\left\{a_{1}, \ldots, a_{p}\right\}$; тем самым, $f \in \mathscr{A}^{0}(\overline{\mathbb{C}} \backslash \Sigma)$. Поскольку в (1) $a_{j}$ и $\alpha_{j}$ - параметры, мы фактически рассматриваем здесь целый класс многозначных аналитических функций (обозначим его $\mathscr{L}$ ), заданных представлением (1). Класс функций $\mathscr{L}$ будем называть классом Лагерра.

Функция $f$ вида (1) удовлетворяет дифференциальному уравнению

$$
A_{p}(z) w^{\prime}+B_{p-2}(z) w=0
$$

где $A_{p}(z)=\prod_{j=1}^{p}\left(z-a_{j}\right), B_{p-2} \in \mathbb{C}_{p-2}[z]-$ полином степени $p-2$, который определяется равенством $B_{p-2}(z)=-A_{p}(z) \sum_{j=1}^{p} \frac{\alpha_{j}}{z-a_{j}}$. Таким образом, функцию $f$ 
вида (1) можно изначально задать в окрестности некоторой точки $z=z_{1} \in \mathbb{C} \backslash \Sigma$ как решение $w=f_{1}$ дифференциального уравнения (2). В таком случае любую другую функцию $w=f_{2}$, которая в окрестности некоторой другой точки $z=z_{2} \neq z_{1}$ удовлетворяет дифференциальному уравнению (2), естественно рассматривать как аналитическое продолжение исходной функции $f_{1}=f$. Такой подход к понятию аналитического продолжения предложен в [55] и [27], [28] для случая дифференциального уравнения произвольного порядка с коэффициентами из поля $\mathbb{C}(z)$. Дифференциальное уравнение $(2)$, так же как и представление (1), задает многозначную аналитическую функцию $f(z)=w(z)$ с помощью конечного числа комплексных параметров - коэффициентов полиномов $A_{p}$ и $B_{p-2}$. Явным представлением (1) многозначная аналитическая функция $f$ определена неоднозначно: для заданной точки $z_{0} \in \overline{\mathbb{C}}$ необходимо зафиксировать определенную ветвь этой функции. Например, для $z=\infty$ в силу условия $\sum_{j=1}^{p} \alpha_{j}=0$ можно положить $f(\infty)=1$. Дифференциальное уравнение $(2)$ содержит еще больший произвол: его нетривиальным решением является любая функция вида $w=$ const $f$, const $\neq 0$, где $f$ задана представлением (1). Тем не менее дифференциальное уравнение типа (2) вполне можно сделать исходным при задании аналитической функции. После этого уже естественно поставить задачу изучения тех или иных свойств аналитической функции непосредственно из уравнения вида (2) или, более общо́, - линейного дифференциального уравнения с рациональными коэффициентами.

Именно на таком подходе к понятию аналитической функции основана так называемая “теория Колчина". В процессе развития теории интегрируемости $^{1}$ линейных однородных дифференциальных уравнений с рациональными коэффициентами из поля $\mathbb{C}(z)$ Э. Колчин [55] в 1959 г. впервые поставил задачу о наилучших ращиональных аппроксимащиях многозначных аналитических функций, которые являются решениями дифференциального уравнения вида

$$
L[w] \equiv 0,
$$

где

$$
L[w]:=w^{(n)}+a_{n-1} w^{(n-1)}+\cdots+a_{1} w^{\prime}+a_{0} w
$$

- линейный дифференциальный оператор порядка $n \in \mathbb{N}$ с коэффициентами $a_{k}$, принадлежащими полю $\mathbb{C}(z)$. Отметим, что Колчин в [55] рассмотрел следующую более общую задачу. Коэффициенты $a_{k} \in \mathbb{k}(z)$ уравнения вида $(3)-$ рациональные функции формальной переменной $z$ с коэффициентами из поля $\mathbb{k}$ характеристики нуль. Решения уравнения (3) рассматривались в класce $\mathbb{k}[[z]]$ формальных степенных рядов. Колчиным ставилась задача о наилучшей рациональной аппроксимации таких решений уравнения (3) рациональными функциями из класса $\mathbb{k}(z)$. "Наилучшая" аппроксимация понималась в локальном смысле: порядок касания ${ }^{2} \nu_{n}(f):=\max \left\{\operatorname{ord}_{z=z_{0}}(f-r): r \in \mathbb{k}_{n}(z)\right\}$ заданного формального ряда $f \in \mathbb{k}[[z]]$ рациональной функцией из класса

\footnotetext{
${ }^{1}$ Точнее говоря, теории неинтегрируемости таких дифференциальных уравнений; подробнее см. монографию А. Г. Хованского [54] и имеющуюся там библиографию.

2 Здесь и далее $\operatorname{ord}_{x=x_{0}} \varphi(x)$ означает порядок нуля функции $\varphi$ в точке $x_{0}$.
} 
$\mathbb{k}_{n}(z):=\{r(z)=p(z) / q(z), p, q \in \mathbb{k}[z], \operatorname{deg} p, \operatorname{deg} q \leqslant n\}$ фиксированного порядка $n$ должен быть максимально возможным. В настоящей работе $\mathbb{k}=\mathbb{C}$ и, тем самым, рассматривается задача о рациональных (из класса $\mathbb{C}_{n}(z)$ ) аппроксимациях аналитических по переменному $z$ решений дифференциального уравнения вида $(3)$, коэффициенты $a_{k}$ которого - рациональные функции из поля $\mathbb{C}(z)$. Отметим, что работа Колчина [55] оказалась тесно связана с так называемыми функциональными аналогами классической теоремы Туэ-Зигеля-Рота и ее дальнейшими обобщениями в теории чисел. Интерес к этой проблематике существует уже многие десятилетия и не ослабевает до сих пор; см. прежде всего [28], [55], [64], [83], [85], [86], [100], а также п. 2.1 ниже.

Таким образом, вполне естественно допустить возможность того, что аналитическая функция $f$ задается как решение линейного дифференциального уравнения с полиномиальными коэффициентами из кольца $\mathbb{C}[z]$, и поставить перед собой задачу: изучить те или иные свойства так заданной аналитической функции через это дифференциальное уравнение, не имея возможности решить это уравнение в явном виде (см. [54]). Так делается в [28], [85], [87], где изучаются свойства приближаемости аналитических функций, заданных как решения дифференциальных уравнений, рациональными функциями. Точнее, следуя Колчину [55], в [28], [49] авторы изучают локальные аппроксимационные свойства локалъно наилучших рациональных аппроксимаций многозначных аналитических функций, заданных как решения уравнения вида $L[w] \equiv 0$, где $L$ - оператор (4) с коэффициентами из поля $\mathbb{C}(z)$. "Локальность" в [28] понимается в смысле максимально возможного касания рациональной функцией фиксированного порядка заданной функции в одной или нескольких точках.

Хорошо известно, что именно аппроксимации Паде ${ }^{3}$ являются локально наилучшими рациональными аппроксимациями заданной аналитической функции $f \in \mathbb{C}[[z]]$ в соответствующем классе рациональных функций.

Настоящая работа посвящена обсуждению только одного вопроса, связанного с изучением свойств аналитической функции, заданной как решение дифференциального уравнения вида $L[w] \equiv 0$, а именно задаче представления такой аналитической функции непрерывной $J$-дробью или, более общо, $T$-дробью. Точнее, речь идет о следующей задаче: где - в какой области или объединении областей - и в каком смысле такое представление справедливо? Очевидно, что речь по существу идет об изучении тех или иных глобальных аппроксимационных свойств локально наилучших рациональных аппроксимаций аналитических функций, т. е. глобальных аппроксимационных свойств аппроксимаций Паде. В этом состоит принципиальное отличие результатов, полученных в рамках теории Колчина, от результатов, вытекающих из теорем Шталя (для классических одноточечных АП; см. п. 2.2) и Буслаева (для многоточечных АП; см. п. 2.3).

Для того чтобы понять, насколько содержательна такая задача и насколько сильно она отличается от задач, решаемых в рамках теории Колчина, вполне естественно рассмотреть класс специальных функций, заданных представлением вида (1). Действительно, такие функции, с одной стороны, задаются явным

\footnotetext{
${ }^{3}$ Напомним, что под АП мы здесь понимаем классические (одноточечные) АП, многоточечные АП и аппроксимации Эрмита-Паде.
} 
представлением (1). С другой стороны, они являются решением дифференциального уравнения вида (2), т. е. весьма частного случая того класса дифференциальных уравнений вида $L[w] \equiv 0$, о которых идет речь в [28], [49], [55] (см. также монографию [54] и [77]). Отметим, что трактовка аналитической функции как решения дифференциального уравнения вида (2) (весьма частного случая общего однородного алгебраического уравнения вида (4)) приобретает особую значимость для теории аппроксимаций Эрмита-Паде (см. ниже п. 2.4), т. е. для той области теории АП, где до сих пор не существует сколько-нибудь общей теоремы о распределении нулей полиномов Эрмита-Паде в классе многозначных аналитических функций с конечным множеством точек ветвления, которая хоть в какой-то степени могла бы быть сравнима с теоремами Шталя и Буслаева (см. [5], [81], [112], а также [75], [76]).

Вполне аналогичным образом можно считать, что аналитическая функция $f$ является алгебраической функцией, т.е. задана алгебраическим уравнением с полиномиальными коэффициентами из $\mathbb{C}[z]$. Соответственно этому возникает задача об изучении тех или иных свойств этой функции $f$ на основе этого алгебраического уравнения (см. [28], [114], а также [54]).

Отметим, что задание аналитической функции с помощью дифференциального уравнения не ограничивается лишь только линейным алгебраическим дифференциальным уравнением вида $L[w] \equiv 0$. Соответствующий пример нелинейного дифференциального уравнения доставляет хорошо известное свободное уравнение Ван дер Поля

$$
\frac{d^{2} U}{d t^{2}}+\varepsilon\left(U^{2}-1\right) \frac{d U}{d t}+U=0,
$$

где $U=U(t ; \varepsilon)$ - величина, связанная с силой тока в электрической цепи, $t-$ время, а физические характеристики самого физического прибора ("генератора") описываются одним "малым" параметром $\varepsilon$. В этом случае частота и амплитуда, соответствующие предельному циклу этого уравнения, оказываются аналитическими функциями параметра $\varepsilon$ (точнее, $\varepsilon^{2}$; подробнее см. [2], [31], [118], а также [3], [16], [17], [34], [52], [53], [80], [95], [101]-[105] в связи с другими возможными приложениями полиномов Эрмита-Паде).

\section{2. Наилучшие рациональные аппроксимации}

Под наилучшими рациональными аппроксимациями в настоящей работе мы понимаем только локально наилучшие. О наилучших рациональных чебышёвских аппроксимациях и соответствующем применении общего GRS-метода см. работу [92] и имеющиеся там ссылки.

2.1. Теория Колчина и функциональные аналоги теоремы Туэ-Зигеля-Рота. Интерес к различным функциональным аналогам классической теоремы Туэ-Зигеля-Рота и ее дальнейшим обобщениям в теории чисел существует уже многие десятилетия и не ослабевает до сих пор (см. прежде всего [55], [85], а также [28], [64], [86], [100], [127]). В последнее время такие результаты связаны с именами Г.В. и Д.В. Чудновских [28], Ч. Ф. Осгуда [83]-[87], В. М. Шмидта [97]--[99], П. Войты [125], [126]. Как правило, 
такие аналоги классических теорем Туэ-Зигеля-Рота и Шмидта для теории чисел связываются, прежде всего, с работой Э. Колчина [55] и рассматриваются как “теория Колчина", которую он сам начал развивать для достаточно общих дифференциальных полей над произвольным полем $\mathbb{k}$ без делителей нуля (см. [28], [55], [85]). В настоящей работе такая теория рассматривается с точки зрения теории аппроксимаций Паде, в качестве основного поля $\mathbb{k}(z)$ мы берем поле рациональных функций $\mathbb{C}(z)$ над полем комплексных чисел $\mathbb{C}$, а в качестве пространства функций - многозначные аналитические функции с конечным множеством особых точек, в частности алгебраические функции и функции, являющиеся решениями линейных однородных алгебраических дифференциальных уравнений. Отметим, что возможности применения метода Гончара-Рахманова-Шталя (GRS-метода, подробнее см. раздел 3), о котором здесь пойдет речь, гораздо шире, чем класс многозначных аналитических функций с конечным множеством особых точек.

Близость теории Колчина к теории аппроксимаций Паде хорошо понимал А. А. Гончар: в обоих случаях речь идет о локально наилучших рациональных аппроксимациях формального степенного ряда или конечного набора таких рядов, различие состоит в рассматриваемых задачах и, как следствие, методах их исследования. Исходя из задач, естественным образом возникавших в теории АП, А.А. Гончар в 1978 г. сформулировал [39] (см. также [6; гл. 1, 6 , п. 6.3]) ряд гипотез, утверждения которых он считал естественным рассматривать как функциональные аналоги теоремы Туэ-Зигеля-Рота. Большая часть этих гипотез теперь доказана (см. прежде всего [28], [110], [111]), однако по крайней мере одна не доказана до сих пор (см. [6; гл. $1, \S 6$, п. 6.3, гипотеза 6.10]). В этой гипотезе речь идет о сильной асимптотике наилучших чебышёвских рациональных аппроксимаций многозначных алгебраических функций; см. также [4], [34], [92].

В трактовке Гончара (см. [39], а также [6; гл. 1, § 6, п. 6.3]) сущность функциональных аналогов теоремы Туэ-Зигеля-Рота состоит в том, что эти результаты являются результатами о структуре возможной лакунарности последовательности порядков касания $\nu_{n}(f), n=1,2, \ldots, \nu_{n}(f):=\max \left\{\operatorname{ord}_{z=z_{0}}(f-r): r \in\right.$ $\left.\mathbb{C}_{n}(z)\right\}$. Или, эквивалентно, это утверждения о нормальности (или возможной ненормальности) индексов в диагональной последовательности АП. Таким образом, это все результаты о некоторых важных, но все-таки вспомогательных свойствах АП, к изучению которых не сводится теория наилучших рациональных аппроксимаций аналитических функций. Содержание замечательных результатов Чудновских [28] по существу состоит в том, что для решений дифференциальных уравнений размер лакун (а тем самым, и размер блоков в таблице Паде) ограничен некоторой эффективной постоянной.

В настоящей работе, в частности, показано, каким образом некоторые функциональные аналоги теоремы Туэ-Зигеля-Рота вытекают в качестве непосредственных следствий из глубоких результатов Г. Шталя и В. И. Буслаева о предельном распределении нулей и полюсов АП для многозначных аналитических функций.

В [55] Колчиным была сформулирована следующая гипотеза (см. [28]). Пусть функция $f$ - решение алгебраического дифференциального уравнения (3), ана- 
литическое $^{4}$ в некоторой точке $z=z_{0} \in \overline{\mathbb{C}}$. Колчин высказал предположение о том, что для всякого положительного $\varepsilon>0$ существует некоторая константа $C=C\left(f, z_{0}, \varepsilon\right)>0$ такая, что для произвольных полиномов $P, Q \in \mathbb{C}[z]$ выполняется неравенство

$$
\underset{z=z_{0}}{\operatorname{ord}}\left(f(z)-\frac{P(z)}{Q(z)}\right)<(2+\varepsilon) \max \{\operatorname{deg} P, \operatorname{deg} Q\}+C,
$$

где $\operatorname{ord}_{x=x_{0}} \varphi(x)$ означает порядок нуля функции $\varphi$ в точке $x_{0}$. В дальнейшем наряду с соотношением (6) стали рассматривать (см. [28], [86]) и следующее более общее соотношение:

$$
\sum_{j \in J} \underset{z=z_{j}}{\operatorname{ord}}\left(f(z)-\frac{P(z)}{Q(z)}\right)<(2+\varepsilon) \max \{\operatorname{deg} P, \operatorname{deg} Q\}+C,
$$

где $J$ - конечное множество индексов, card $J<\infty$. При этом предполагалось, что элемент $f$ - голоморфная в каждой точке $z_{j}, j \in J$, функция и $L[f](z) \equiv 0$ для некоторой окрестности $U_{j}$ каждой точки $z_{j}$. В дальнейшем под гипотезой Колчина стали понимать и более сильное утверждение, а именно возможность того, что в (6) и (7) величина $\varepsilon$ равна 0 , а постоянная $C$ является "эффективной”. Ясно, что соотношение (6) связано со свойствами классических аппроксимаций Паде для некоторых классов аналитических функций, а соотношение (7) - со свойствами многоточечных аппроксимаций Паде (см. пп. $2.2,2.3)$. Аналогичные соотношения рассматриваются в [28] и для аппроксимаций Эрмита-Паде. В работе Чудновских [28] доказана справедливость соотношений (6) и (7) в классе решений алгебраических дифференциальных уравнений произвольного порядка и алгебраических функций, причем в наиболее сильном варианте: для $\varepsilon=0$ и с некоторой эффективной постоянной $C$. Одна из целей настоящей работы - весьма просто получить соотношения (6) и $(7)$ с $\varepsilon>0$ и некоторой неэффективной постоянной $C$, но зато в существенно более широком классе функций, чем в [28], [55] и [86], а именно в классе многозначных аналитических функиии, имеющи в $\overline{\mathbb{C}}$ конечное множество точек ветвления (подробнее см. ниже пп. 2.2, 2.3). При нашем подходе соотношение (6) является непосредственным следствием теоремы Шталя [110], a (7) - непосредственным следствием теоремы Буслаева [21]. А именно, того факта, что в рамках этих теорем для соответствующих полиномов Паде $P_{n}, Q_{n}$ степени $n$ существует предельное распределение нулей и

$$
\frac{1}{n} \chi\left(P_{n}\right), \frac{1}{n} \chi\left(Q_{n}\right) \stackrel{*}{\rightarrow} \lambda^{\mathrm{eq}}, \quad n \rightarrow \infty,
$$

где $\lambda^{\text {eq }}$ - соответствующая равновесная мера (для тривиального внешнего поля $\psi(z) \equiv 0$ в случае Шталя и внешнего поля $\psi(z)=V^{-\nu}(z)$ в случае Буслаева, где $\nu$ - единичный положительный заряд, сосредоточенный в конечном числе точек $z_{1}, \ldots, z_{m}$ - точек $m$-интерполяции исходной функции $\left.f\right)$. Здесь $\chi(Q)-$ мера, "считающая" нули полинома $Q \in \mathbb{C}[z]$ с учетом их кратностей, см. (25).

\footnotetext{
${ }^{4}$ Как обычно, под аналитичностью функции в точке понимается аналитичность функции в некоторой окрестности этой точки.
} 
В дальнейшем будет рассматриваться только случай, когда в (6) и (7) $\operatorname{deg} P=\operatorname{deg} Q$, тем самым, речь пойдет только о диагональных аппроксимациях Паде.

ЗАмечАниЕ 1. Возможно рассматривать и более общую ситуацию, когда задано нормированное дифференциальное поле $\mathscr{T}$, в котором для любых его элементов $a, b \in \mathscr{T}$ определена операция $\delta$ такая, что $\delta(a b)=\delta(a) b+a \delta(b)$, $\delta(a+b)=\delta(a)+\delta(b)$, и эта операция согласована с нормой $|\cdot| \geqslant 0,|a b|=|a||b|$, в следующем смысле. Существуют некоторые положительные величины $c_{1}$ и $c_{2}$ такие, что $c_{1}|a| \leqslant|\delta a| \leqslant c_{2}|a|$ для всех $|a|<1, a \in \mathscr{T}$. В этой связи см. [64], [85], а также [46], [47].

ЗАмечАниЕ 2. Обсудим коротко связь асимптотических свойств АП и так называемого аппроксимативного спектра Шмидта; см. [64]. Пусть $z_{0}=\infty$. Для подходящих 5 дробей $P_{n} / Q_{n}$ имеем:

$$
\left|f-\frac{P_{n}}{Q_{n}}\right|=\left|\frac{1}{Q_{n}}\right|^{1+\operatorname{deg} Q_{n+1} / \operatorname{deg} Q_{n}} .
$$

Аппроксимативный спектр Шмидта $[64 ; \S 4]$ определяется формулой

$$
\operatorname{Spec}(f)=\left\{1+\frac{\operatorname{deg} Q_{n+1}}{\operatorname{deg} Q_{n}}, n=1,2, \ldots\right\}^{\prime} .
$$

Пусть $f \in \mathscr{A}^{0}(\overline{\mathbb{C}} \backslash \Sigma)$. Тогда непосредственно из теоремы Шталя (см. п. 2.2) вытекает, что $\operatorname{Spec}(f)=\{2\}$. Если же $\mathfrak{f}=\left\{\left(f_{1}, z_{1}\right), \ldots,\left(f_{m}, z_{m}\right)\right\}$ - мультиросток, где $f_{j} \in \mathscr{A}^{0}(\overline{\mathbb{C}} \backslash \Sigma)$ и $f_{j} \in \mathscr{H}\left(z_{j}\right)$, то из теоремы Буслаева (см. п. 2.3) получаем равенство $\operatorname{Spec}(\mathfrak{f})=\{2\}$.

2.2. Аппроксимации Паде: теория Шталя. Пусть $\Sigma=\left\{a_{1}, \ldots, a_{p}\right\}-$ конечное множество точек в комплексной плоскости $\mathbb{C}$. Через $\mathscr{A}(\overline{\mathbb{C}} \backslash \Sigma)$ обозначим множество всех функций, аналитических в области $\mathbb{C} \backslash \Sigma$, т. е. каждая из функций $f \in \mathscr{A}(\overline{\mathbb{C}} \backslash \Sigma)$ голоморфна в произвольной точке $z \notin \Sigma$ и аналитически продолжается из этой точки вдоль любого пути, не пересекающего множество $\Sigma$. Класс функций, аналитических, но не голоморфных в области $\overline{\mathbb{C}} \backslash \Sigma$, обозначим через $\mathscr{A}^{0}(\overline{\mathbb{C}} \backslash \Sigma)$, т. е. $\mathscr{A}^{0}(\overline{\mathbb{C}} \backslash \Sigma):=\mathscr{A}(\overline{\mathbb{C}} \backslash \Sigma) \backslash \mathscr{H}(\overline{\mathbb{C}} \backslash \Sigma)$. Тем самым, для произвольной функции $f \in \mathscr{A}^{0}(\overline{\mathbb{C}} \backslash \Sigma)$ хотя бы одна из точек $a_{j} \in \Sigma$ является ее точкой ветвления.

Теоремы о представлении аналитических функций вида (1) непрерывной $J$-дробью или, более общо, $T$-дробью, пока не удается доказать иначе как в виде следствия из общих теорем Шталя (для $J$-дробей) и Буслаева (для $T$-дробей и более общих непрерывных дробей) о сходимости соответствующих диагональных АП в классе всех многозначных аналитических функций с конечным множеством точек ветвления. Поскольку функции вида (1) удовлетворяют дифференциальному уравнению вида (2), соответствующие полиномы Паде также удовлетворяют однородному алгебраическому дифференциальному уравнению 2-го порядка (см. ниже (14)). Однако хотя коэффициенты этого уравнения - полиномы фиксированной степени, они зависят от номера соответствующей АП.

\footnotetext{
${ }^{5}$ Имеются в виду как $J$-дроби, так и $T$-дроби и многоточечные АП.
} 
Точнее, эти полиномы содержат так называемые акцессорные параметры, зависящие от номера $n$ соответствующего полинома Паде. Асимптотическое поведение этих параметров при $n \rightarrow \infty$ априори неизвестно. Как для классических АП, так и для двухточечных АП это поведение удается изучить только опираясь соответственно на теорию Шталя и теорию Буслаева. В результате в некоторых случаях удается найти и асимптотические формулы для соответствующих полиномов Паде; см. [57], [74], [82], ср. также [70], [71].

Напомним, что, поскольку $a_{j}, \alpha_{j}, j=1, \ldots, p,-$ параметры, мы фактически рассматриваем здесь целый класс многозначных аналитических функций $\mathscr{L}$, заданных представлением вида (1). Все функции класса Лагерра $\mathscr{L}$ удовлетворяют дифференциальному уравнению вида (2).

Вопрос о представлении таких функций рядом Лорана решается весьма просто. А именно, пусть $f \in \mathscr{L}$, тогда $f$ голоморфна в бесконечно удаленной точке $z=\infty$ и, следовательно, разлагается в точке $z=\infty$ в сходящийся ряд Лорана:

$$
f(z)=\sum_{k=0}^{\infty} \frac{c_{k}}{z^{k}}, \quad|z|>\max _{j=1, \ldots, p}\left|a_{j}\right| .
$$

Зафиксируем ветвь функции $f$ условием $f(\infty)=1=c_{0}$. Тогда из представления (1) коэффициенты $c_{k}$ ряда Лорана (9) однозначно определяются по рекуррентным формулам, для которых нетрудно найти явное выражение.

Задача о разложении функции $f, f(\infty)=1$, в непрерывную $J$-дробь оказалась гораздо сложнее.

В 1885 г. Лагерр [62] рассмотрел задачу о разложении функции вида (1) в непрерывную $J$-дробь и в этой связи естественным образом пришел к задаче об асимптотике знаменателей $Q_{n} n$-х подходящих дробей $J_{n}=P_{n} / Q_{n}$ для непрерывной $J$-дроби, которые оказались неэрмитово ортогональными многочленами. В частности, Лагерр рассмотрел (см. также [30], [67], [81]) эту задачу для функции вида

$$
f(z)=\prod_{j=1}^{3}\left(z-a_{j}\right)^{\alpha_{j}}
$$

где $\prod_{j=1}^{3} \alpha_{j}=0, \alpha_{j} \in \mathbb{C} \backslash \mathbb{Z}, f(\infty)=1$, и три точки $a_{1}, a_{2}, a_{3}$ находятся в общем положении, в частности не лежат на одной прямой. Оказалось, что знаменатели $Q_{n}$ подходящих дробей $J_{n}=P_{n} / Q_{n}$, соответствующих непрерывной $J$-дроби, определяемой соотношениями

$$
\begin{aligned}
f(z) & =\prod_{j=1}^{3}\left(z-a_{j}\right)^{\alpha_{j}}=1+\sum_{k=1}^{\infty} \frac{c_{k}}{z^{k}}=1+\frac{c_{1}}{z-\widehat{b}_{1}+f_{1}(z)} \\
& =1+\frac{\widehat{a}_{1}^{2}}{z-\widehat{b}_{1}-\frac{\widehat{a}_{2}^{2}}{z-\widehat{b}_{2}+f_{2}(z)}} \simeq 1+\frac{\widehat{a}_{1}^{2}}{z-\widehat{b}_{1}-\frac{\widehat{a}_{2}^{2}}{z-\widehat{b}_{2}-\cdots}}=: J(z),
\end{aligned}
$$

обладают свойством (неэрмитовой) ортогональности. Точнее, справедливо соотношение

$$
\oint_{\Gamma} Q_{n}(\zeta) \zeta^{k} f(\zeta) d \zeta=0, \quad k=0, \ldots, n-1
$$


где $\Gamma$ - произволъный замкнутый контур, отделяющий точки $a_{1}, a_{2}, a_{3}$ от бесконечно удаленной точки $z=\infty$. Отметим, что монические ортогональные

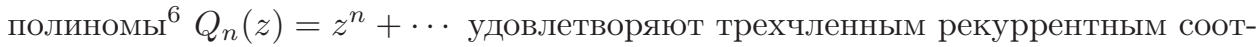
ношениям

$$
Q_{n}(z)=\left(z-\widehat{b}_{n}\right) Q_{n-1}(z)-\widehat{a}_{n}^{2} Q_{n-2}(z), \quad n=1,2, \ldots
$$

$Q_{-1}(z) \equiv 0, Q_{0}(z) \equiv 1, Q_{1}(z)=z-\widehat{b}_{1}$. В связи с задачей о представлении функции $f$ непрерывной $J$-дробью (11), т. е. задачей об асимптотическом поведении соответствующих подходящих дробей $J_{n}$, естественным образом возник вопрос, возможно ли непосредственно из соотношений ортогональности (12) получить описание асимптотического поведения полиномов $Q_{n}$, или, эквивалентно, знаменателей рациональных функций $J_{n}$. В случае положительного ответа на этот вопрос, Лагерром было бы получено решение задачи о представлении функции (9) непрерывной $J$-дробью, т. е. решение задачи о равенстве $f(z)=J(z)$. Более точно, был бы получен ответ на вопрос, в какой области комплексной плоскости функция (9) представляется непрерывной $J$-дробью. Отметим, что в [62] (см. также [76], [82], [89]) Лагерром было выведено также следующее дифференциальное уравнение 2-го порядка, решениями которого являются полиномы $P_{n}$ и функции $Q_{n} f$ и $Q_{n} f-P_{n}$ (ср. (15)):

$$
A_{3}(z) \Pi_{n, 1}(z) w^{\prime \prime}+\Pi_{n, 3}(z) w^{\prime}+\Pi_{n, 2}(z) w=0
$$

где $A_{3}(z)=\prod_{j=1}^{3}\left(z-a_{j}\right)$, а $\Pi_{n, k} \in \mathbb{C}_{k}[z], k=1,2,3,-$ полиномы степени ровно $k$.
Точнее,

$$
\begin{array}{ll}
\Pi_{n, 1}(z)=z-z_{n}, & \Pi_{n, 2}(z)=-n(n+1)\left(z-b_{n}\right)\left(z-v_{n}\right), \\
\Pi_{n, 3}(z)=\left(z-z_{n}\right) B_{2}(z)-A_{3}(z), & B_{2}(z)=A_{3}^{\prime}(z) \frac{f^{\prime}(z)}{f(z)} .
\end{array}
$$

Дифференциальное уравнение (14) - это алгебраическое дифференциальное уравнение 2-го порядка с полиномиальными коэффициентами фиксированной степени. Но эти коэффициенты зависят от номера $n$. Точнее говоря, коэффициенты уравнения (14) содержат три акцессорных параметра $z_{n}, b_{n}, v_{n}$, асимптотическое поведение которых при $n \rightarrow \infty$ заранее неизвестно, и большой параметр $n(n+1)$ при свободном члене. Несмотря на полученные соотношение ортогональности (12) и дифференциальное уравнение (14) Лагерру не удалось решить на первый взгляд простую задачу об асимптотическом поведении полиномов $Q_{n}$. Это удалось сделать Дж. Наттоллу [82] в 1986 г. (см. также [74], [76]), но только лишь после того, как Г. Шталь [107]-[111] (см. также [6], [122]) полностью решил задачу о предельном распределении нулей полиномов Паде, соответствующих произвольной многозначной аналитической функции с конечным множеством точек ветвления на римановой сфере.

\footnotetext{
${ }^{6} \mathrm{~B}$ случае общего положения $\operatorname{deg} Q_{n}=n$ при всех $n$.
} 
Хорошо известно (см. [81], [110], [111]), что соотношения ортогональности вида (12) естественным образом возникают в теории аппроксимаций Паде. Это вполне естественно, поскольку подходящие дроби $J_{n}$ для непрерывной $J$-дроби, соответствующей произволъному (находящемуся в общем положении) ряду Лорана $f \in \mathscr{H}(\infty)$, совпадают с $n$-й диагональной аппроксимацией Паде, построенной по этому ряду Лорана следующим образом. Для произвольного $n \in \mathbb{N}$ определим полиномы Паде $P_{n, 0}, P_{n, 1} \in \mathbb{C}_{n}[z], \operatorname{deg} P_{n, 0}, \operatorname{deg} P_{n, 1} \leqslant n, P_{n, 1} \not \equiv 0$, из соотношения

$$
H_{n}(z):=\left(P_{n, 0}+P_{n, 1} f\right)(z)=O\left(\frac{1}{z^{n+1}}\right), \quad z \rightarrow \infty .
$$

Такие полиномы всегда существуют, но определяются соотношением (15) не единственным образом. Однако рациональная функция $P_{n, 0} / P_{n, 1}$ порядка $\leqslant n$ определена уже однозначно; функция $[n / n]_{f}:=-P_{n, 0} / P_{n, 1}$ называется диагональной аппроксимацией Паде функции $f \in \mathscr{H}(\infty)$ порядка $n$ (или $n$-диагональной аппроксимацией Паде). При этом справедливо равенство $J_{n}=[n / n]_{f}$. В частности, для функции вида (10) полиномы $P_{n, 1}$ удовлетворяют тем же неэрмитовым соотношениям ортогональности (12). Хорошо известно, что общие ортогональные многочлены были открыты П. Л. Чебышёвым [26] в 1855 г. именно в рамках теории непрерывных $J$-дробей. Этот факт отражен в монографии Г. Сегё [120; п. 3.5] следующим образом: "Исторически ортогональные многочлены впервые рассматривались в теории непрерывных дробей. Эта связь очень важна и явлется одной из возможных отправных точек при исследовании ортогональных многочленов...".

Отметим, что при таком подходе при разложении в непрерывную дробь функции $f \in \mathscr{L}$ вида

$$
f(z)=\left(\frac{z-1}{z+1}\right)^{\alpha}, \quad f(\infty)=1, \quad \alpha \in\left(-\frac{1}{2}, \frac{1}{2}\right), \quad \alpha \neq 0,
$$

естественным образом возникают полиномы Якоби $P^{(\alpha,-\alpha)}(z)$ с параметрами $\alpha \in(-1 / 2,1 / 2) \backslash\{0\}$ и $\beta=-\alpha$, ортогональные на отрезке $\Delta:=[-1,1]$ с весом $((1-x) /(1+x))^{\alpha}($ см. $[120])$.

Несмотря на то что класс Лагерра $\mathscr{L}$ состоит из многозначных аналитических функций весьма специального вида, фактически заданных явными формулами вида (1), задача об асимптотических свойствах соответствующих полиномов Паде, или, эквивалентно, полиномов, удовлетворяющих соотношениям ортогональности (12), оказалась весьма характерной с точки зрения общей теории АП. А именно, даже для случая функции $f$ вида (10) Лагерру не удалось решить задачу об асимптотическом поведении соответствующих ортогональных многочленов - знаменателей диагональных АП. Как уже отмечалось, это удалось сделать Дж. Наттоллу [82] в 1986 г. (см. также [74]) только после того, как Г. Шталь полностью решил (см. [114]) задачу о предельном распределении нулей полиномов Паде, соответствующих произвольной многозначной аналитической функции с конечным множеством точек ветвления на римановой сфере. Краеугольным камнем теории Шталя является его утверждение о том, что для каждой многозначной аналитической функции $f \in \mathscr{H}(\infty)$ с конечным 
множеством $^{7}$ точек ветвления на римановой сфере существует единственный (с точностью до произвольного множества нулевой емкости) компакт $S=S(f)$, обладающий определенным свойством "симметрии" (или, иначе, $S$-свойством; см. (18)) и такой, что: $S$ состоит из конечного числа аналитических дуг, множество $D:=\overline{\mathbb{C}} \backslash S$ является областью и исходная функция голоморфно продолжается в область $D, f \in \mathscr{H}(D)$. На основе этого результата в теории Шталя доказывается, что предельное распределение нулей полиномов Паде существует и совпадает с равновесной робеновской мерой $\lambda=\lambda_{S}^{\text {rob }}$ для компакта $S$, т. е. $-\int_{S} \log |z-\zeta| d \lambda(\zeta) \equiv$ const $=\gamma_{S}, z \in S, \gamma_{S}-$ постоянная Робена для $S$. Компакт Шталя $S$ однозначно характеризуется ${ }^{8}$ свойством минимальной емкости в классе всех компактов $\Gamma$ таких, что $\Gamma=\partial G, G$ - область, $G \ni \infty$ и $f \in \mathscr{H}(G)$, т. e.

$$
\operatorname{cap} S=\min _{\Gamma=\partial G} \operatorname{cap} \Gamma,
$$

а вышеупомянутое свойство симметрии состоит в том, что для любой точки $z \in S^{\circ}$ выполняется равенство

$$
\frac{\partial g_{D}(z, \infty)}{\partial n^{+}}=\frac{\partial g_{D}(z, \infty)}{\partial n^{-}}
$$

где $S^{\circ}$ - объединение открытых аналитических дуг, замыкания которых составляют $S, g_{D}(z, \infty)$ - функция Грина для области Шталя $D \ni \propto$ с особенностью в бесконечно удаленной точке, $\partial / \partial n^{ \pm}$- нормальные производные к компакту Шталя $S$, взятые в точке $z \in S^{\circ}$ с противоположных сторон от $S$. В силу равенства $g_{D}(z, \infty)=\gamma_{S}-V^{\lambda}(z)$ соотношение (18) эквивалентно соотношению

$$
\frac{\partial V^{\lambda}}{\partial n^{+}}(z)=\frac{\partial V^{\lambda}}{\partial n^{-}}(z), \quad z \in S^{\circ} .
$$

Для случая классических полиномов Якоби, соответствующих функции (16), компакт Шталя $S$ совпадает с единичным отрезком: $S=\Delta=[-1,1]$. Для случая обобщенных полиномов Якоби, соответствующих функции (10) (напомним, что три точки $a_{1}, a_{2}, a_{3}$ находятся в общем положении и, в частности, не лежат на одной прямой), компакт Шталя $S$ совпадает с континуумом Чеботарёва $\mathrm{C}\left(a_{1}, a_{2}, a_{3}\right)$ (см. [61], а также рис. 1 и 2$)$. В этом случае компакт $S$ состоит из критических траекторий квадратичного дифференциала

$$
-\frac{z-v}{A_{3}(z)} d z^{2}>0, \quad A_{3}(z):=\prod_{j=1}^{3}\left(z-a_{j}\right),
$$

соединяющих точки ветвления $a_{j}$, являющиеся простыми полюсами квадратичного дифференциала (20), с так называемой точкой Чеботарёва $z=v$ - простым нулем дифференциала (20). Точка Чеботарёва $v$ - это трансцендентный

\footnotetext{
${ }^{7}$ Результаты Шталя носят гораздо более общий характер и справедливы в классе многозначных аналитических функций, множество особенностей которых имеет нулевую емкость.

${ }^{8}$ с точностью до произвольного компакта нулевой емкости.
} 


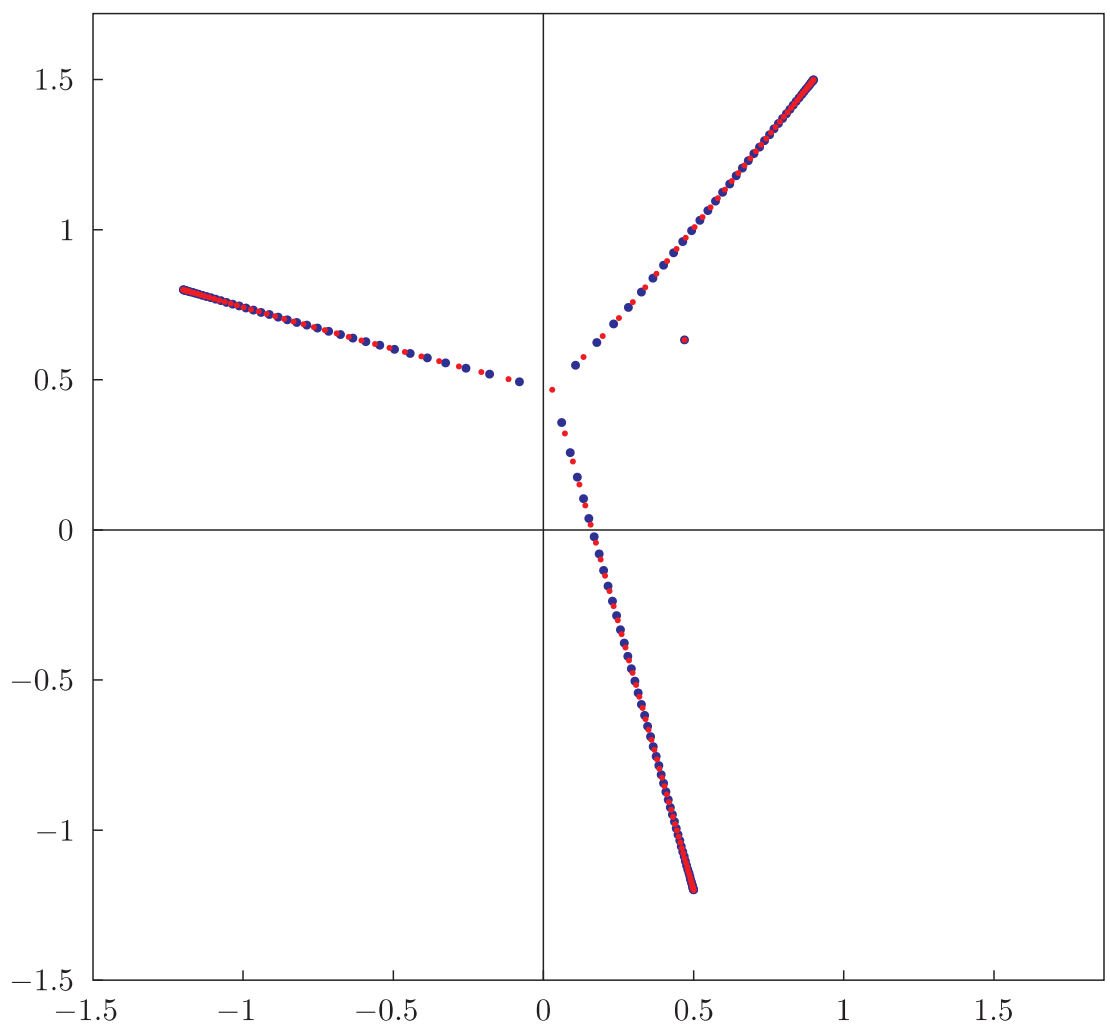

Рис. 1. Нули и полюсы диагональных аппроксимаций Паде $[130 / 130]_{f}$ функции $f(z)=[(z+1.2-i \cdot 0.8)(z-0.9-i \cdot 1.5)(z-0.5+i \cdot 1.2)]^{-1 / 3}$. При фиксированном $n=130$ распределение нулей и полюсов соответствует электростатической модели Рахманова [91]. На рисунке имеется одна пара Фруассара. Так как род соответствующей римановой поверхности Шталя равен 1 , не может быть больше одной пары Фруассара. Поведение этой пары Фруассара при $n \rightarrow \infty$ подчиняется уравнению из работы [82].

параметр задачи, она однозначно определяется из условия, что все периоды абелева интеграла

$$
\int^{z} \sqrt{\frac{\zeta-v}{A_{3}(\zeta)}} d \zeta
$$

чисто мнимые. Тем самым, функция

$$
\operatorname{Re} \int_{a_{1}}^{z} \sqrt{\frac{\zeta-v}{A_{3}(\zeta)}} d \zeta
$$

- однозначная гармоническая функция на двулистной эллиптической римановой поверхности $\mathfrak{R}_{2}: w^{2}=(z-v) A_{3}(z)$, компакт Чеботарёва-Шталя определя- 


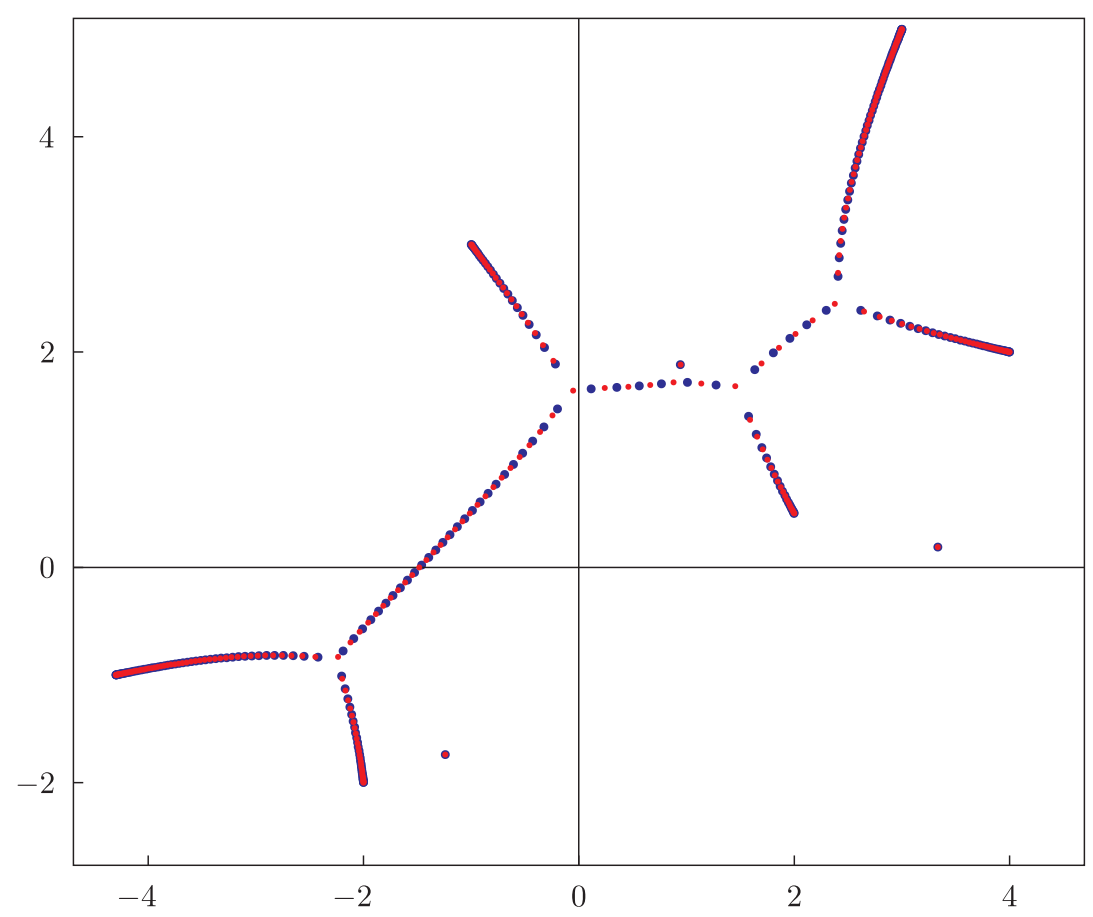

Рис. 2. Нули и полюсы диагональных аппроксимаций Паде $[266 / 266]_{f}$ функции $f(z)=[(z+4.3+i)(z-2-i \cdot 0.5)(z+2+i \cdot 2)(z+1-i \cdot 3)(z-4-i \cdot 2) \times$ $(z-3-i \cdot 5)]^{-1 / 6}$. В пределе при $n \rightarrow \infty$ нули и полюсы диагональных аппроксимаций Паде $[n / n]_{f}$ должны распределяться в соответствии с теоремой Шталя [114]. При заданном $n=266$ эти нули и полюсы распределяются на римановой сфере в соответствии с электростатической моделью Рахманова [91]. Поскольку для заданной функции $f$ род соответствующей гиперэллиптической поверхности Шталя равен 4 , при каждом $n$ может быть не более 4 пар Фруассара. При заданном $n=266$ на рисунке наблюдаются 3 пары Фруассара.

ется соотношением

$$
S=\left\{z \in \mathbb{C}: \operatorname{Re} \int_{a_{1}}^{z} \sqrt{\frac{\zeta-v}{A_{3}(\zeta)}} d \zeta=0\right\},
$$

а функция

$$
g(z):=\operatorname{Re} \int_{a_{1}}^{z} \sqrt{\frac{\zeta-v}{A_{3}(\zeta)}} d \zeta
$$

является функцией Грина $g_{D}(z, \infty)$ для области Шталя $D=\overline{\mathbb{C}} \backslash S$.

Таким образом, решить задачу о распределении нулей полиномов Паде для функций весьма специального вида (10) удается только в рамках общей теоремы Шталя, полученной им для произвольной многозначной аналитической функции с конечным множеством точек ветвления на римановой сфере. 


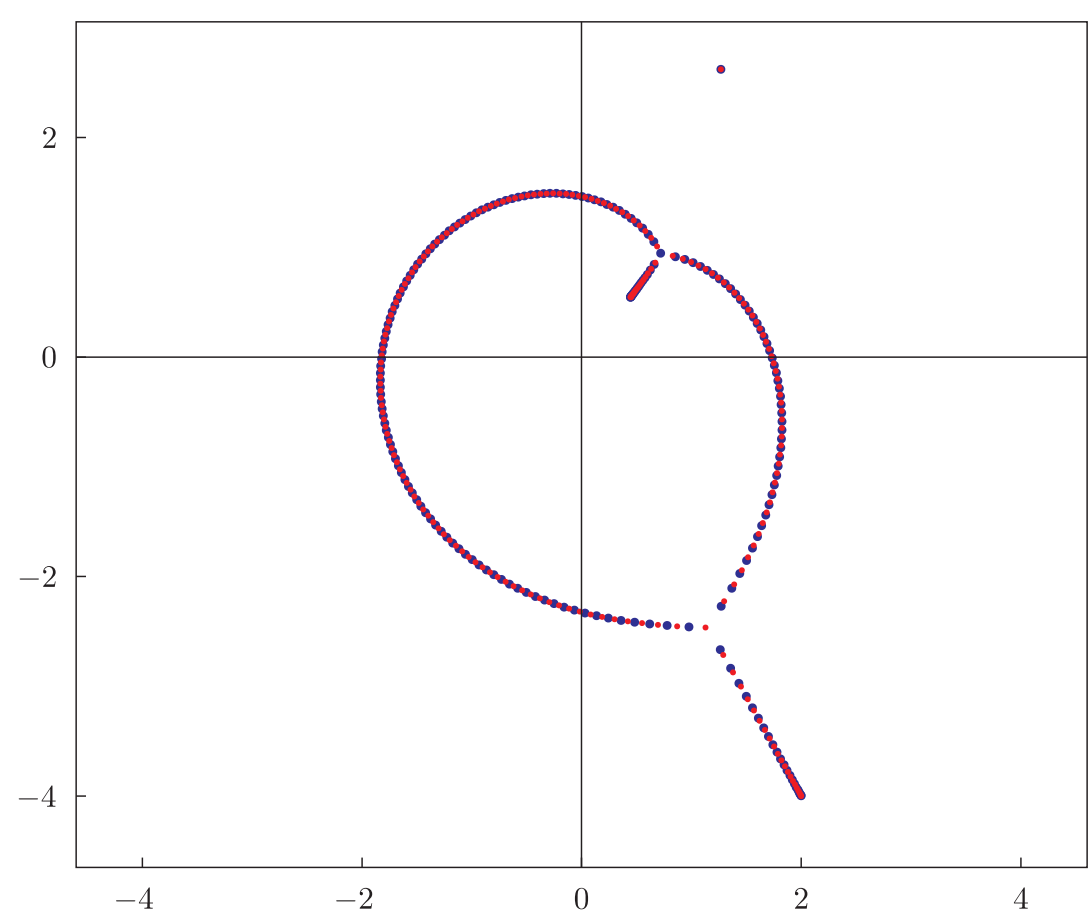

Рис. 3. Численное распределение нулей и полюсов двухточечных аппроксимаций Паде [199/199] для многозначной функции $f(z)=$ $\left(\frac{z-a_{1}}{z-a_{2}}\right)^{1 / 4}$, где $a_{1}=0.9-i \cdot 1.1, a_{2}=0.1+i \cdot 0.2$, для случая, когда выбраны две "существенно различные" ветви $f_{0}=\left(\frac{z-a_{1}}{z-a_{2}}\right)^{1 / 4}$ и $f_{\infty}=-\left(\frac{z-a_{1}}{z-a_{2}}\right)^{1 / 4}$ функции $f$. Почти все нули (синие точки) и полюсы (красные точки) в совокупности моделируют численно компакт Буслаева. Но, кроме того, есть одна пара Фруассара, поведение которой при $n \rightarrow \infty$ подчиняется уравнению из работы [57].

В силу сказанного полиномы Паде для функций вида (1) естественно рассматривать как обобщение классических полиномов Якоби (см. [74], [82]). Другой возможный класс обобщенных полиномов Якоби связан с так называемыми двухточечными полиномами Паде; см. [56], [57], а также рис. 3. Изучение соответствующих асимптотических свойств таких полиномов основано на теореме Буслаева - двухточечном аналоге теоремы Шталя (см. [21], [22], а также п. 2.3); отметим, что полученный Буслаевым в [21] результат носит более общий характер: он справедлив для $m$-точечных АП в классе всех многозначных аналитических функций с конечным множеством особых точек на римановой сфере; см. п. 2.3. Наконец, еще одно возможное обобщение полиномов Якоби связано с полиномами Эрмита-Паде 1-го рода для набора трех функций $\left[1, f, f^{2}\right]$, где функция $f$ задана представлением (16) при $\alpha \in(-1 / 2,1 / 2), \alpha \neq 0$; подробнее см. [75] и [76]. 
Для произвольной меры $\mu, \operatorname{supp} \mu \Subset \mathbb{C}$, через $V^{\mu}(z)$ обозначим логарифмический потенциал $\mu$ :

$$
V^{\mu}(z):=-\int \log |z-t| d \mu(t)
$$

а через $V_{*}^{\mu}(z)$ - соответствующий сферически нормированный потенциал:

$$
V_{*}^{\mu}(z):=\int_{|\zeta| \leqslant 1} \log \frac{1}{|z-\zeta|} d \mu(\zeta)+\int_{|\zeta|>1} \log \frac{1}{|1-z / \zeta|} d \mu(\zeta) .
$$

Для произвольного полинома $Q \in \mathbb{C}[z], Q \not \equiv 0$, через

$$
\chi(Q)=\sum_{\zeta: Q(\zeta)=0} \delta_{\zeta}
$$

обозначим ассоциированную меру, "считающую” нули полинома $Q$ с учетом их кратностей, а через $Q^{*}$ - соответствующий сферически нормированный полином:

$$
Q^{*}(z)=\prod_{\substack{\zeta:|\zeta| \leqslant 1 \\ Q(\zeta)=0}}(z-\zeta) \cdot \prod_{\substack{\zeta:|\zeta|>1 \\ Q(\zeta)=0}}\left(1-\frac{z}{\zeta}\right)
$$

Теорема Шталя (см. [110]). Пусть $f \in \mathscr{H}(\infty), f \in \mathscr{A}^{0}(\overline{\mathbb{C}} \backslash \Sigma), \operatorname{Card} \Sigma<\infty$. Пусть $D=D(f)$ - область Шталя для функиии $f, S=S(f)=\partial D$ - соответствующий компакт Шталя, $[n / n]_{f}=-P_{n, 0} / P_{n, 1}-n$-я диагональная аппроксимачия Паде функиии $f$ (в бесконечно удаленной точке). Тогда справедливы следующие утверждения:

1) существует предельное распределение нулей полиномов Паде $P_{n, j}, j=$ 0,1, при этом

$$
\frac{1}{n} \chi\left(P_{n, j}\right) \stackrel{*}{\rightarrow} \lambda_{S}^{\mathrm{rob}}, \quad n \rightarrow \infty, \quad j=0,1,
$$

где $\lambda_{S}^{\mathrm{rob}}$ - равновесная робеновская мера для компакта Шталя $S: V^{\lambda_{S}^{\mathrm{rob}}}(z) \equiv$ const $=\gamma_{S}, z \in S, \gamma_{S}-$ постоянная Робена для $S$;

2) диагональные АП сходятся по емкости ${ }^{9} \kappa$ функиии $f$ внутри (т.е. на компактных подмножествах) области D:

$$
[n / n]_{f}(z) \stackrel{\text { cap }}{\longrightarrow} f(z), \quad n \rightarrow \infty, \quad z \in D ;
$$

скорость сходимости в (27) характеризуется соотношением

$$
\left|\left(f-[n / n]_{f}\right)(z)\right|^{1 / n} \stackrel{\text { cap }}{\longrightarrow} e^{-2 g_{D}(z, \infty)}, \quad n \rightarrow \infty, \quad z \in D,
$$

где $g_{D}(z, \infty)$ - функиия Грина для области D.

Непосредственно из теоремы Шталя получаем, что

$$
\frac{\operatorname{deg} P_{n, j}}{n} \rightarrow 1, \quad n \rightarrow \infty, \quad j=0,1 .
$$

Из (29) и (28) вытекает справедливость гипотезы Колчина (6) для произволъной функции $f \in \mathscr{A}^{0}(\overline{\mathbb{C}} \backslash \Sigma)$.

\footnotetext{
${ }^{9}$ Определение сходимости по емкости см. в [44], [110], [114].
} 
2.3. Многоточечные аппроксимации Паде: теория Буслаева. Пусть $f_{j} \in \mathscr{A}^{0}(\overline{\mathbb{C}} \backslash \Sigma), \operatorname{Card} \Sigma<\infty, j=1, \ldots, m$. Пусть все точки $z_{1}, \ldots, z_{m} \in$ $\mathbb{C} \backslash \Sigma$ попарно различны и $f_{j} \in \mathscr{H}\left(z_{j}\right), j=1, \ldots, m$. Пусть $P_{n}, Q_{n} \in \mathbb{C}_{n}[z]$, $P_{n}, Q_{n} \not \equiv 0,-$ полиномы степеней $\leqslant n$ такие, что выполняется соотношение

$$
\left(Q_{n} f_{j}-P_{n}\right)\left(z_{j}\right)=O\left(\left(z-z_{j}\right)^{n_{j}}\right), \quad z \rightarrow z_{j}, \quad j=1, \ldots, m,
$$

где $\sum_{j=1}^{m} n_{j}=2 n+1, n_{j} \in \mathbb{Z}_{+}, j=1, \ldots, m$. Соотношением (30) полиномы $P_{n}, Q_{n}$ определены не однозначно, однако рациональная функция $B_{n}=$ $P_{n} / Q_{n}$ соотношениями (30) определена уже единственным образом и называется многоточечной (или $m$-точечной) аппроксимацией Паде для множества $\mathfrak{f}=\left\{f_{1}, \ldots, f_{m}\right\}$ функций $f_{j} \in \mathscr{A}^{0}(\overline{\mathbb{C}} \backslash \Sigma)$, аналитических в соответствующих точках $z_{j}$, или, короче говоря, для $m$-ростка ${ }^{10}\left\{\left(f_{1}, z_{1}\right), \ldots,\left(f_{m}, z_{m}\right)\right\}$, состоящего из $m$ аналитических функций $f_{j}$, голоморфных в точках $z_{j}, j=1, \ldots, m$. Если $z_{j}=\infty$ при некотором $j \in\{1, \ldots, m\}$, то соответствующие соотношения (30) и (32) необходимо изменить (см. [21], [22]). При фиксированном $m$ $m$-росток $\mathfrak{f}=\left\{\left(f_{1}, z_{1}\right), \ldots,\left(f_{m}, z_{m}\right)\right\}$ мы иногда будем коротко называть мультиростком.

В (30) функции $f_{1}, \ldots, f_{m}$ - это, вообще говоря, различные аналитические функции, ни одна из которых, скажем, $f_{j}$, не может быть получена как аналитическое продолжение другой функции $f_{k} \in \mathfrak{f}, k \neq j$, вдоль некоторого пути $\gamma \subset \overline{\mathbb{C}} \backslash \Sigma$. Однако если для некоторого алгебраического дифференциального оператора конечного порядка $L[w]$ вида (4) при всех $j=1, \ldots, m$ выполняется соотношение $L\left[f_{j}\right](z) \equiv 0$ для $z \in U_{j}\left(z_{j}\right)$, то в соответствии с подходом работ [27]-[30], [49], [55] все функции $f_{j} \in \mathfrak{f}$ естественно рассматривать как различные ветви одной и той же многозначной аналитической функции $f$, для которой $L[f](z) \equiv 0, z \notin \Sigma$. Отметим, что трактовка аналитической функции как решения дифференциального уравнения вида (2) (весьма частного случая общего однородного алгебраического уравнения вида (4)) приобретает особую значимость для теории аппроксимаций Эрмита-Паде (см. ниже п. 2.4), т. е. той области теории АП, где до сих пор не существует сколько-нибудь общей теоремы о распределении нулей полиномов Эрмита-Паде в классе многозначных аналитических функций с конечным множеством точек ветвления, которая хоть в какой-то степени могла бы быть сравнима с теоремами Шталя и Буслаева (см. [5], [81], [112], а также [75], [76]).

В случае общего положения соотношения (30) эквивалентны соотношениям

$$
\left(f_{j}-B_{n}\right)(z)=O\left(\left(z-z_{j}\right)^{n_{j}}\right), \quad z \rightarrow z_{j}, \quad j=1, \ldots, m .
$$

Предположим, что в соотношении (30) $\frac{n_{j}}{n} \rightarrow 2 p_{j}$ при $n \rightarrow \infty$ и $\sum_{j=1}^{m} p_{j}=1$, $p_{j} \geqslant 0, j=1, \ldots, m$. В теории Буслаева утверждается (см. [21]-[23], а также [20]), что (в невырожденном случае) существует единственный ${ }^{11}$ компакт

\footnotetext{
${ }^{10}$ Как обычно, росток многозначной аналитической функции $f$ мы обозначаем той же буквой $f$, что и саму функцию.

${ }^{11}$ с точностью до произвольного множества нулевой емкости.
} 
$F=F_{\text {Вus }}$, который является $S$-кривой ${ }^{12}$, взвешенной во внешнем поле, порожденном отрицательным единичным зарядом $-\nu, \nu=\sum_{j=1}^{m} p_{j} \delta_{z_{j}}$, и сосредоточенном в точках $z_{1}, \ldots, z_{m}$. Этот компакт $F$ обладает следующими свойствами: $F$ состоит из конечного числа аналитических дуг; дополнение $\overline{\mathbb{C}} \backslash F$ состоит из конечного числа областей $D_{j} \ni z_{j}, \overline{\mathbb{C}} \backslash F=\bigcup_{j=1}^{m} D_{j}$; каждая из функций $f_{j} \in \mathscr{H}\left(z_{j}\right)$ является голоморфной (т. е. аналитической и однозначной) функцией в соответствующей области $D_{j}, f_{j} \in \mathscr{H}\left(D_{j}\right)$; если при некоторых $k \neq j$ две области совпадают, $D_{j}=D_{k}$, то совпадают и соответствующие функции, $f_{k}=f_{j}$; компакт $F$ обладает свойством "симметрии" во внешнем поле $V_{*}^{-\nu}$, а именно, справедливо соотношение

$$
\frac{\partial\left(V^{\beta_{F}}-V_{*}^{\nu}\right)}{\partial n^{+}}(z)=\frac{\partial\left(V^{\beta_{F}}-V_{*}^{\nu}\right)}{\partial n^{-}}(z), \quad z \in F^{\circ},
$$

где $\beta_{F} \in M_{1}(F)$ - единственная единичная равновесная мера для компакта $F$, находящегося во внешнем поле $V_{*}^{-\nu}$ (т. е. для $\beta_{F}$ выполняется тождество $V^{\beta_{F}}(z)-V_{*}^{\nu}(z) \equiv$ const $\left.=w_{F}, z \in F\right) ; F^{\circ}$ - объединение всех открытых дуг, замыкания которых составляют $F ; \partial / \partial n^{ \pm}$- нормальные производные к $F$, взятые в точке $z \in F^{\circ}$ с противоположных сторон от $F$. Отметим, что при фиксированном мультиростке $\mathfrak{f}=\left\{\left(f_{1}, z_{1}\right), \ldots,\left(f_{m}, z_{m}\right)\right\}$ компакт $F$ в существенной степени зависит от выбора чисел ("весов") $p_{j} \geqslant 0, \sum_{j=1}^{m} p_{j}=1$. Тем самым, "оптимальное" разбиение римановой сферы на области $D_{j}$ также зависит от выбора чисел $p_{j}$.

Подчеркнем, что, так же как и в теории Шталя, существование взвешенной во внешнем поле $S$-кривой $F$ является ключевым в теории Буслаева. После того как существование такой $S$-кривой установлено, вопрос о соответствующей равновесной мере $\beta_{F}$ решается просто: $\beta_{F}=\widetilde{\nu}=\mathfrak{b}_{F}(\nu)$ - выметание меры $\nu$ из открытого множества $D=\bigcup_{j=1}^{m} D_{j}$ на его границу $\partial D=F$. Отметим, что граница $\partial D_{j}$ каждой из областей $D_{j}$ содержит открытую дугу $\gamma_{j}^{\circ} \subset \partial D_{j}$ такую, что $\gamma_{j}^{\circ} \cap \partial D_{k}=\varnothing$ при $k \neq j$. Аналогично теории Шталя, $S$-свойство $(32)$ взвешенной $S$-кривой $F$ эквивалентным образом можно записать в следующем виде (ср. (18), (19)):

$$
\frac{\partial\left(\sum_{j=1}^{m} p_{j} g_{D_{j}}\left(z, z_{j}\right)\right)}{\partial n^{+}}=\frac{\partial\left(\sum_{j=1}^{m} p_{j} g_{D_{j}}\left(z, z_{j}\right)\right)}{\partial n^{-}}, \quad z \in F^{\circ},
$$

где $g_{D_{j}}\left(z, z_{j}\right)$ - функция Грина для области $D_{j}$ (как обычно, при $z \in D_{k} \neq D_{j}$ полагаем $\left.g_{D_{j}}\left(z, z_{j}\right) \equiv 0\right)$.

ТеОрема БУСЛАеВа (см. [21], [22]). Пусть $\mathfrak{f}=\left\{\left(f_{1}, z_{1}\right), \ldots,\left(f_{m}, z_{m}\right)\right\}-$ мультиросток, состоящий из т аналитических функиий $f_{j}$ таких, что $f_{j} \in$ $\mathscr{H}\left(z_{j}\right), f_{j} \in \mathscr{A}^{0}(\overline{\mathbb{C}} \backslash \Sigma), j=1, \ldots, m$, где $\operatorname{Card} \Sigma<\infty$ и все точки $z_{1}, \ldots, z_{m} \in \mathbb{C}$

\footnotetext{
${ }^{12}$ По поводу этого понятия см. [44], [60], [91], [114].
} 
попарно различны. Пусть $p_{j} \geqslant 0, \sum_{j=1}^{m} p_{j}=1$ компакт $F=F\left(\mathfrak{f} ; p_{1}, \ldots, p_{m}\right)-$ соответствующий компакт Буслаева, обладающий $S$-свойством во внешнем поле $V_{*}^{-\nu}(z), \nu=\sum_{j=1}^{m} \delta_{z_{j}}, \bigcup_{j=1}^{m} D_{j}=\overline{\mathbb{C}} \backslash F-$ соответствующее оптимальное разбиение римановой сферь, $D_{j} \ni z_{j}, f \in \mathscr{H}\left(D_{j}\right)$. Пусть $B_{n}=P_{n} / Q_{n}$, $B_{n}(z)=B_{n}\left(z ; \mathfrak{f} ; p_{1}, \ldots, p_{m}\right)$ - соответствующая диагональная $m$-точечная аппроксимация Паде к мультиростку f. Тогда справедливы следующие утверждения:

1) существует предельное распределение нулей и полюсов т-точечных АП $B_{n}=P_{n} / Q_{n}$, а именно,

$$
\frac{1}{n} \chi\left(P_{n}\right), \frac{1}{n} \chi\left(Q_{n}\right) \stackrel{*}{\rightarrow} \beta_{F}, \quad n \rightarrow \infty ;
$$

2) т-точечные АП сходятся по емкости к функции $f_{j}$ внутри области $D_{j}$, $j=1, \ldots, m$ :

$$
B_{n}(z) \stackrel{\text { cap }}{\longrightarrow} f_{j}(z), \quad n \rightarrow \infty, \quad z \in D_{j}, \quad j=1, \ldots, m ;
$$

скорость сходимости в (35) при $j=1, \ldots$, m характеризуется соотношением

$$
\left|f_{j}(z)-B_{n}(z)\right|^{1 / n} \stackrel{\text { cap }}{\longrightarrow} \exp \left\{-2 \sum_{k=1}^{m} p_{k} g_{D_{k}}\left(z, z_{k}\right)\right\}, \quad n \rightarrow \infty, \quad z \in D_{j} .
$$

Непосредственно из теоремы Буслаева вытекает справедливость гипотезы Колчина (7) для произвольного $m$-ростка $\mathfrak{f}=\left\{f_{1}, \ldots, f_{m}\right\}$, где $f_{j} \in \mathscr{A}^{0}(\overline{\mathbb{C}} \backslash \Sigma)$, Card $\Sigma<\infty$.

2.4. Аппроксимации Эрмита-Паде. Пусть $f_{1}, f_{2} \in \mathscr{A}^{0}(\overline{\mathbb{C}} \backslash \Sigma)$, $\operatorname{Card} \Sigma<\infty$, $f_{1}, f_{2} \in \mathscr{H}(\infty)$. Всюду в дальнейшем предполагается, что функции $f_{1}, f_{2}$ рационально независимы с функцией $f_{0} \equiv 1$. Положим $\mathbb{P}_{n}:=\mathbb{C}_{n}[z]$.

Для произвольного $n \in \mathbb{N}$ определим полиномы Эрмита-Паде 1-го рода $Q_{n, 0}, Q_{n, 1}, Q_{n, 2} \in \mathbb{P}_{n}, \operatorname{deg} Q_{n, j} \leqslant n, Q_{n, j} \not \equiv 0$, из соотношения

$$
R_{n}(z):=\left(Q_{n, 0} \cdot 1+Q_{n, 1} f_{1}+Q_{n, 2} f_{2}\right)(z)=O\left(\frac{1}{z^{2 n+2}}\right), \quad z \rightarrow \infty ;
$$

функция $R_{n}$ называется функцией остатка. Соотношением (37) полиномы $Q_{n, j}$ определяются неоднозначно, но их отношения, например, $Q_{n, 1} / Q_{n, 2}$, определены уже единственным образом. Поскольку функции $1, f_{1}, f_{2}$ рационально независимы, функция остатка не равна нулю тождественно, $R_{n} \not \equiv 0$. Более подробно о свойствах полиномов Эрмита-Паде и соответствующих аппроксимаций Эрмита-Паде см. прежде всего [17], [68], [79], [81], [112], а также [5], [7], [10], [41], [121]. Отметим, что мы рассматриваем здесь только полиномы Эрмита-Паде 1-го рода, определенные соотношением (37). Так называемые полиномы Эрмита-Паде 2-го рода, или, иначе говоря, совместно ортогональные полиномы, связаны с полиномами Эрмита-Паде 1-го рода некоторыми формальными соотношениями (см. [81; §2, формула (2.1.3)], [37], [122]). Однако 
здесь мы этой связи не обсуждаем и полиномы Эрмита-Паде 2-го рода не рассматриваем.

Вопрос об асимптотических свойствах полиномов Эрмита-Паде 1-го рода ${ }^{13}$ даже для набора $\left[1, f_{1}, f_{2}\right]$ из трех многозначных аналитических функций изучен в гораздо меньшей степени, чем для классических и многоточечных АП. Принципиальные трудности возникают здесь уже для (простейших с точки зрения теории классических АП или двухточечных АП) аналитических функций. Так обстоит дело, например, для набора из трех функций $\left[1, f, f^{2}\right]$, где функция $f$ имеет вид

$$
f(z)=\prod_{j=1}^{3}\left(z-a_{j}\right)^{\alpha_{j}}, \quad 2 \alpha_{j} \in \mathbb{C} \backslash \mathbb{Z}, \quad \sum_{j=1}^{3} \alpha_{j}=0,
$$

при условии, что точки $a_{1}, a_{2}, a_{3}$ находятся в общем положении, в частности не лежат на одной прямой. За исключением симметричного случая, когда точки $a_{1}, a_{2}, a_{3}$ являются вершинами правильного треугольника (см. [81]), для этого набора до сих пор не удается доказать существование предельного распределения нулей соответствующих полиномов Эрмита-Паде; подробнее см. [11], [12], [119]. Отметим, что при вышеуказанных условиях пара функций $f, f^{2}$ образует систему Никишина; по поводу определения, сходимости в вещественном случае и основных свойств таких систем см. прежде всего оригинальную работу Е. М. Никишина [78], а также [9], [36], [45], [65].

Другой случай не менее характерен с точки зрения демонстрации тех сложностей, которые испытывает общая теория распределения нулей полиномов Эрмита-Паде для многозначных аналитических функций с конечным множеством точек ветвления. Рассмотрим набор из трех функций $\left[1, f_{1}, f_{2}\right]$, где функции $f_{1}$ и $f_{2}$ заданы представлениями

$$
\begin{aligned}
& f_{1}(z)=\left(\frac{z-a}{z+1}\right)^{1 / 2}=\frac{1}{\pi} \int_{-1}^{a} \sqrt{\frac{a-x}{x+1}} \frac{d x}{x-z}+1, \quad z \notin \Delta_{1}:=[-1, a], \\
& f_{2}(z)=\left(\frac{z-1}{z+a}\right)^{1 / 2}=\frac{1}{\pi} \int_{-a}^{1} \sqrt{\frac{1-x}{x+a}} \frac{d x}{x-z}+1, \quad z \notin \Delta_{2}:=[-a, 1]
\end{aligned}
$$

$a$ - некоторый параметр, $a \in(0,1)$. В представлениях (39) и (40) выбрана такая ветвь функции $(\cdot)^{1 / 2}$, что $f_{1}(z), f_{2}(z) \rightarrow 1$ при $z \rightarrow \infty$; под $\sqrt{\cdot}$ здесь и всюду в дальнейшем понимается арифметическое значение корня, т. е. $\sqrt{x^{2}}=x$ при $x \in \mathbb{R}_{+}$. Ясно, что $f_{1}, f_{2}$ - марковские функции, носителями которых являются соответственно отрезки $\Delta_{1}=[-1, a]$ и $\Delta_{2}=[-a, 1]$. Каждая из функций $f_{1}$ и $f_{2}$ имеет по паре особенностей - ветвлений 2-го порядка, расположенных в точках $-1, a$ и $-a, 1$, т. е. два множества особых точек $\{-1, a\}$ и $\{-a, 1\}$, в отличие от рассмотренного выше случая $\left[1, f, f^{2}\right]$, не пересекаются между собой. Поскольку $a \in(0,1)$, то $\Delta_{1} \cap \Delta_{2}=[-a, a] \neq \varnothing$, но $\Delta_{1} \not \subset \Delta_{2}, \Delta_{2} \not \subset \Delta_{1}$. Тем самым, пара функций $f_{1}, f_{2}$ не является ни системой Анжелеско, ни системой Никишина. Численные эксперименты, проведенные в [51] (см. также [50]), показали, что в этом на первый взгляд весьма простом случае, в котором все исходные данные

\footnotetext{
13Это высказывание относится и к полиномам Эрмита-Паде 2-го рода.
} 
вещественные, при описании предельного распределения нулей соответствующих полиномов Эрмита-Паде неизбежно возникнут $S$-кривые, расположенные уже в комплексной плоскости (разумеется, симметрично относительно вещественной оси; подробнее см. [51]). В теории распределения нулей полиномов Эрмита-Паде пока нет общих результатов, с помощью которых можно было бы объяснить экспериментально полученные в [51] численные результаты о распределении нулей полиномов Эрмита-Паде. Отметим недавнюю работу [13], в которой аналогичная задача изучается для случая полиномов Эрмита-Паде 2-го рода.

Таким образом, даже для (простейших с точки зрения общей теории классических АП и многоточечных АП) наборов $\left[1, f, f^{2}\right]$ и $\left[1, f_{1}, f_{2}\right]$ из трех многозначных аналитических функций задача характеризации соответствующих $S$-кривых в терминах ассоциированной (и пока неизвестной даже в плане формальной постановки) теоретико-потенциальной задачи равновесия оказывается до сих пор не решенной.

Пусть теперь в представлении (1) точки $a_{j}$ и параметры $\alpha_{j}$ удовлетворяют следующим дополнительным условиям:

$$
p=2 q, \quad a_{j}=e_{j} \in \mathbb{R}, \quad e_{1}<\cdots<e_{2 q}, \quad \alpha_{j}=(-1)^{j} \alpha,
$$

где $2 \alpha \in \mathbb{R} \backslash \mathbb{Z}$. Тем самым, аналитическая функция $f$ задана представлением

$$
f(z)=\prod_{j=1}^{q}\left(\frac{z-e_{2 j-1}}{z-e_{2 j}}\right)^{\alpha},
$$

в котором точки $e_{j}$ и параметр $\alpha$ удовлетворяют вышеуказанным условиям. Очевидно, что функция $f$ по-прежнему удовлетворяет дифференциальному уравнению вида (2).

В настоящей работе мы изучаем задачу о предельном распределении нулей полиномов Эрмита-Паде 1-го рода для набора трех функций $\left[1, f, f^{2}\right]$, где функция $f$ задана представлением (41), а точки $e_{j}$ и параметр $\alpha$ удовлетворяют вышеуказанным условиям. Всюду в дальнейшем класс таких функций мы будем обозначать через $\mathscr{L}_{E}, E:=\bigsqcup_{j=1}^{q}\left[e_{2 j-1}, e_{2 j}\right]$. Выбор такого на первый взгляд весьма частного набора точек ветвления $e_{j}$ и одинаковых значений параметра $\alpha$ объясняется ниже (см. замечание 3 ). Случай $q=1$ был рассмотрен в [75], [76]. В этом случае функция $f$ имеет вид $f(z)=((z+1) /(z-1))^{\alpha}$ и соответствующие полиномы Эрмита-Паде $Q_{n, j}$ естественно рассматривать как аналог классических полиномов Якоби. Для произвольного $q>1$ соответствующие полиномы Эрмита-Паде $Q_{n, j}$ естественно рассматривать как обобщение полиномов Ахиезера [1], ортогональных на нескольких отрезках (см. соотношение (69), а также работу [81]).

С учетом вышесказанного исследуемая в настоящей работе задача о предельном распределении нулей полиномов Эрмита-Паде 1-го рода для набора из трех функций $\left[1, f, f^{2}\right]$, где функция $f$ задана представлением (41), является вполне актуальной. Отметим, что в оригинальной работе Е. М. Никишина [78] (см. также [45], [101]-[105]) рассматривалась, вообще говоря, более общая (по постановке) задача. А именно, был изучен набор из $m \geqslant 3$ марковских функций 
$\left[1, f_{1}, \ldots, f_{m-1}\right]$ таких, что функции $f_{1}, \ldots, f_{m-1}$ образуют систему Никишина. Напомним, что это, в частности, означает, что носителем всех марковских функций $f_{j}=\widehat{\mu}_{j}$ является одно и то же множество - объединение конечного числа отрезков, расположенных на вещественной прямой. Предельное распределение нулей соответствующих полиномов Эрмита-Паде 1-го рода было охарактеризовано в [78] в терминах некоторой теоретико-потенциальной задачи равновесия, вполне аналогичной задаче, впервые рассмотренной А. А. Гончаром и Е.А. Рахмановым [41] в 1981 г. для случая, когда функции $f_{1}, \ldots, f_{m-1}$ образуют систему Анжелеско (см. также [40], [43]) . Введенная Е. М. Никишиным в [78] теоретико-потенциальная задача равновесия вполне характеризуется так называемой матрицей взаимодействия Никишина. Для $m=3$ и рассматриваемого здесь набора $\left[1, f, f^{2}\right]$ эта матрица Никишина имеет вид $\left(\begin{array}{cc}2 & -1 \\ -1 & 2\end{array}\right)$. В отличие от этого рассматриваемая здесь теоретико-потенциальная задача равновесия, в терминах которой характеризуется предельное распределение нулей полиномов Эрмита-Паде $Q_{n, j}$ для набора функций $\left[1, f, f^{2}\right]$, является скалярной (см. ниже (49), а также замечание 3 ).

Отметим, что переход в (41) к комплексным точкам ветвления $e_{j}$ и к различным комплексным параметрам $\alpha_{j}$ вместо одного $\alpha$ представлял принципиальную трудность даже для случая классических АП и двухточечных АП и не поддавался исследованию до общих теорем Шталя и Буслаева соответственно.

Зафиксируем теперь росток функции $f \in \mathscr{L}_{E}$ условием $f(\infty)=1$. Заметим, что мы вполне могли бы исходить не из явного представления (41), а непосредственно из дифференциального уравнения (2). В силу условия $f(\infty)=1$ и представления (41) имеем

$$
f(z)=\sum_{k=0}^{\infty} \frac{c_{k}}{z^{k}}, \quad \text { где } c_{k} \in \mathbb{R} .
$$

Поэтому стандартное определение (37) полиномов Эрмита-Паде 1-го рода для набора из трех аналитических функций $\left[1, f, f^{2}\right]$ можно уточнить следующим образом. Пусть $Q_{n, 0}, Q_{n, 1} Q_{n, 2} \in \mathbb{R}_{n}[z], Q_{n, 2} \not \equiv 0,-$ полиномы степеней $\leqslant n$, удовлетворяющие соотношению

$$
R_{n}(z):=\left(Q_{n, 0} \cdot 1+Q_{n, 1} f+Q_{n, 2} f^{2}\right)(z)=O\left(\frac{1}{z^{2 n+2}}\right), \quad z \rightarrow \infty .
$$

Как и раньше, такие полиномы всегда существуют, определены не однозначно, но их отношения, например $Q_{n, 1} / Q_{n, 2}$, оказываются уже однозначно определенными рациональными функциями порядка $\leqslant n$. Функция $R_{n}-$ функция остатка. Из условий $e_{1}<\cdots<e_{2 q}$ и $2 \alpha \in \mathbb{R} \backslash \mathbb{Z}$ вытекает, что $f$ и $f^{2}$ - марковские функции, образующие обобщенную систему Никишина (см. [33], [78]). Тем самым, набор $\left[1, f, f^{2}\right]$ невырожден (см. [33], [65]). Следовательно, $R_{n}(z) \not \equiv 0$. Более того, в правой части соотношения (42) порядок нуля может быть больше $2 n+2$ только на фиксированное число, зависящее от $q, e_{j}$ и $\alpha$, а степени полиномов $Q_{n, j}$ могут отличаться от $n$ только на некоторое не зависящее от $n$ число, т. е. $\operatorname{deg} Q_{n, j} \geqslant n-m$, где $m \in \mathbb{N}$ не зависит от $n$. 
Поскольку пара $f, f^{2}$ образует систему Никишина, из результатов работы [65] вытекает, что "почти все" нули полиномов $Q_{n, j}$, кроме $o(n)$ из них, принадлежат множеству $F:=\overline{\mathbb{R}} \backslash E$, где $E=\bigsqcup_{j=1}^{q}\left[e_{2 j-1}, e_{2 j}\right]$. Кроме того, рациональные функции $Q_{n, j} / Q_{n, 2}, j=0,1$, обладают определенными интерполяционными свойствами и сходятся внутри области $G:=\overline{\mathbb{C}} \backslash F$ по $\sigma_{1}$-мере Хаусдорфа. Однако о скорости этой сходимости в [65] ничего не говорится. Одна из целей настоящей работы - характеризация скорости такой сходимости.

Зафиксированный соотношением $f(\infty)=1$ росток $f \in \mathscr{H}(\infty)$ функции $f$, заданной представлением (41), определяет функцию $f$ как голоморфную (т. е. однозначную аналитическую) функцию в области Шталя $D:=\overline{\mathbb{C}} \backslash E$ (относительно бесконечно удаленной точки, т. е. $\left.D=D_{\infty}(f)\right)$. Кроме того, этот росток естественным образом порождает на множестве $\bigsqcup_{j=1}^{q}\left(e_{2 j-1}, e_{2 j}\right)$ семейство ростков $\tilde{f}_{j}(x), x \in\left(e_{2 j-1}, e_{2 j}\right)=: E_{j}^{\circ}, j=1, \ldots, q$, многозначных аналитических функций по следующему правилу:

$$
\widetilde{f}_{j}(x):=f^{+}(x)+f^{-}(x), \quad x \in\left(e_{2 j-1}, e_{2 j}\right),
$$

где под $f^{+}(x)$ при $x \in\left(e_{2 j-1}, e_{2 j}\right)$ понимаются предельные значения функции $f(z), f \in \mathscr{H}(D)$, при стремлении $z \rightarrow x$ из верхней полуплоскости: $f^{+}(x):=$ $f(x+i \cdot 0)$; аналогичный смысл придается и $f^{-}(x): f^{-}(x):=f(x-i \cdot 0)$. Очевидно, что каждая из функций $\widetilde{f}_{j}, j=1, \ldots, q$, удовлетворяет дифференциальному уравнению $(2)$. Тем самым, все функции $\widetilde{f}_{j}$ являются ветвями одной и той же многозначной аналитической функции $f$, заданной дифференциальным уравнением (2) с помощью конечного числа комплексных параметров - полиномиальных коэффициентов уравнения (2). Отметим, что, с точностью до нетривиальной мультипликативной константы, все функции $\tilde{f}_{j}$ являются ветвями многозначной аналитический функции $f$, заданной представлением (41). Таким образом, каждая из этих ветвей получается из заданного в точке $z=\infty$ ростка в результате его аналитического продолжения вдоль некоторых путей, непересекающих точки $e_{1}, \ldots, e_{2 q}$, и последующего умножения на некоторую нетривиальную постоянную. Нетрудно увидеть, что, в силу представления (41), при $2 \alpha \in \mathbb{R} \backslash \mathbb{Z}$ каждая из функций $\tilde{f}_{j}$ имеет нетривиальный скачок в лакунах $\left(e_{2 j-2}, e_{2 j-1}\right)$ и $\left(e_{2 j}, e_{2 j+1}\right)$, смежных с исходным интервалом $\left(e_{2 j-1}, e_{2 j}\right)$. При этом любые две соседние функции, скажем, $\tilde{f}_{j}$ и $\tilde{f}_{j+1}$, являются непосредственными аналитическими продолжениями друг друга как через верхнюю, так и через нижнюю полуплоскость. Тем самым, в рассматриваемой ситуации естественным образом возникает функция $\tilde{f}$, голоморфная в области $G:=\overline{\mathbb{C}} \backslash F \not \supset \infty,\left.\widetilde{f}\right|_{E_{j}^{\circ}}=\widetilde{f}_{j}, j=1, \ldots, m$.

Эта функция также удовлетворяет дифференциальному уравнению (2) и, таким образом, является аналитическим продолжением исходной функции $f$, $f \in \mathscr{H}(D), f(\infty)=1$, в смысле, обсуждаемом в разделе 1.

Конструкция (42), фактически определяющая полиномы Эрмита-Паде, является естественным обобщением конструкции (15) для полиномов Паде. Тем самым, полиномы Паде $P_{n, 0}, P_{n, 1}$ для функции $f$ - это полиномы Эрмита-Паде 
для набора $[1, f]$. Рациональная функция $[n / n]_{f}:=-P_{n, 0} / P_{n, 1}$ имеет максимальный порядок касания с заданной функцией $f$ в точке $z=\infty$. Из общей теоремы Шталя вытекает, что для функции $f$ вида (1) существует предельное распределение нулей полиномов Паде $P_{n, j}$, которое совпадает с равновесной (робеновской) мерой компакта $E:=\bigsqcup_{j=1}^{q}\left[e_{2 j-1}, e_{2 j}\right]$ :

$$
\frac{1}{n} \chi\left(P_{n, j}\right) \rightarrow \lambda_{E}^{\mathrm{rob}}, \quad V_{E}^{\lambda^{\mathrm{rob}}}(x) \equiv \text { const }, \quad x \in E .
$$

Напомним, что для $E=[-1,1]$

$$
d \lambda_{E}^{\mathrm{rob}}=d \lambda^{\mathrm{cheb}}=\frac{1}{\pi} \frac{d x}{\sqrt{1-x^{2}}} .
$$

В силу теоремы Шталя (см. п. 2.2) диагональные АП $[n / n]_{f}$ сходятся по емкости к функции $f, f \in \mathscr{H}(D)$, внутри области Шталя $D$ :

$$
[n / n]_{f}(z) \stackrel{\text { cap }}{\longrightarrow} f(z), \quad z \in D, \quad n \rightarrow \infty .
$$

Принципиальное отсутствие равномерной сходимости диагональных АП в области Шталя $D$ следует уже из классического результата Дюма [35] 1908 г. Такой сходимости нет уже в случае одной лакунъ. Это вызвано наличием так называемых дуплетов Фруассара [38] (см. рис. 1, 2 и 3) или, иначе говоря, совместных пар так называемых "ложных" нулей и "ложных" полюсов диагональных АП, которые не соответствуют ни нулям, ни полюсам, ни другим особенностям исходной функции $f$. Их число связано прежде всего с родом соответствующей двулистной канонической римановой поверхности Шталя (см. [59], [113]), они не имеют предела при $n \rightarrow \infty$, но зато в каждой такой паре в пределе нуль и полюс бесконечно близки друг к другу. При переходе от $n$ к $n+1$ такая пара сдвигается на "почти" фиксированное расстояние (измеряемое в соответствующей метрике) и в “типичном случае" оказывается всюду плотной на римановой сфере. Например, для эллиптической функции (т. е. функции рода $g=1)$, рассмотренной Дюма, в типичном случае асимптотическое поведение пары "ложный нуль-ложный полюс" соответствует всюду плотной обмотке тора. Поскольку в силу теоремы Шталя при $n \rightarrow \infty$ "почти все" нули и полюсы диагональных АП за возможным исключением $o(n)$ из них притягиваются к компакту Шталя, вопрос о так называемой силъной асимптотике полиномов Паде напрямую связан с задачей о полном описании асимптотического поведения всех нулей и полюсов таких АП. Эта задача фактически состоит из двух частей (см. [81] и [113]). Сначала необходимо доказать, что для заданной многозначной функции $f$ таких пар Фруассара только конечное (зависящее от этой функции) число. Затем, уже зная это число, нужно описать их асимптотическое поведение в подходящих терминах. Последняя задача оказывается эквивалентной проблеме обращения Якоби [106], [128], поставленной на ассоциированной с функцией $f$ канонической гиперэллиптической поверхности Шталя (см. [14], [57], [74], [81], [82], [116], [117]). 
Переход в (41) к комплексным точкам ветвления $e_{j}$ и к различным комплексным параметрам $\alpha_{j}$ вместо одного $\alpha$ даже для классических АП потребовал разработки принципиально новых методов исследования для решения задачи о предельном распределении нулей полиномов Паде; см. п. 2.2.

Положим $f_{1}(z):=f(z), z \in D_{1}$, где $D_{1}:=D=\overline{\mathbb{C}} \backslash E, E:=\bigsqcup_{j=1}^{q}\left[e_{2 j-1}, e_{2 j}\right]$; тем самым, $f_{1} \in \mathscr{H}\left(D_{1}\right), f_{1}(\infty)=1$. Пусть $e_{1}=-1, e_{2 q}=1$; тем самым, $\widehat{E}=\operatorname{conv} E=[-1,1]$.

Положим $D_{2}=G:=\overline{\mathbb{C}} \backslash F, F:=\overline{\mathbb{R}} \backslash E$. Пусть функция $f_{2}$ задана на $E^{\circ}$ представлением

$$
f_{2}(x):=-2 \cos (\alpha \pi) \prod_{j=1}^{k-1}\left(\frac{x-e_{2 j-1}}{x-e_{2 j}}\right)^{\alpha} \cdot\left(\frac{x-e_{2 k-1}}{e_{2 k}-x}\right)^{\alpha} \cdot \prod_{j=k+1}^{q}\left(\frac{e_{2 j-1}-x}{e_{2 j}-x}\right)^{\alpha},
$$

$x \in\left(e_{2 k-1}, e_{2 k}\right), k=1, \ldots, q$. Тогда $f_{2}$ продолжается с $E^{\circ}$ в $\overline{\mathbb{C}} \backslash\left\{e_{1}, \ldots, e_{2 q}\right\}$ как многозначная аналитическая функция. Соотношением (46) фиксируется ветвь функции $f_{2}$, голоморфная в области $D_{2}, f_{2} \in \mathscr{H}\left(D_{2}\right)$. Очевидно, что функция $f_{2}$ также удовлетворяет дифференциальному уравнению (2). Тем самым, в соответствии с подходом раздела $1, f_{2}$ - это другая ветвь исходной аналитической функции $f_{1}$, голоморфная в другой области $D_{2} \neq D_{1}$.

Пусть $g_{E}(\zeta, z), z, \zeta \in D_{1},-$ функция Грина для области $D_{1}$ с особенностью в точке $\zeta=z$ и $g_{F}(\zeta, z), z, \zeta \in D_{2},-$ функция Грина для области $D_{2}$ с особенностью в точке $\zeta=z$. Введем

$$
G_{F}^{\mu}(z):=\int_{E} g_{F}(x, z) d \mu(x), \quad z \in D_{2},
$$

- гринов (относительно области $D_{2}$ ) потенциал (положительной борелевской) единичной меры $\mu$ с носителем на $E, \mu \in M_{1}(E)$.

Основной результат настоящей работы составляет следующая теорема.

Теорема 1. Пусть $\alpha \in(-1 / 2,1 / 2), \alpha \neq 0, e_{1}<\cdots<e_{2 q}$, бункиия $f_{1}=f$ задана соотношением (41), $f_{1} \in \mathscr{H}\left(D_{1}\right)$, функиия $f_{2}$ задана соотношением (46), $f_{2} \in \mathscr{H}\left(D_{2}\right)$. Пусть $Q_{n, j}=Q_{n, j}(z ; f)$ - полиномь Эрмита-Паде для набора $\left[1, f, f^{2}\right]$. Тогда справедливы следующие утверждения:

1) все нули полиномов $Q_{n, 0}, Q_{n, 1} u Q_{n, 2}$, за возможным исключением фиксированного числа, не зависящего от $n$, принадлежат множеству $F$; существует предельное распределение нулей полиномов $Q_{n, j}$, которое совпадает c (единственной) единичной мерой $\eta_{F} \in M_{1}(F)$, имеющей носитель на $F$ и равновесной для смешанного потенииала $3 V_{*}^{\mu}(z)+G_{E}^{\mu}(z)$ во внешнем поле $\psi(z):=3 g_{E}(z, \infty):$

$$
\frac{1}{n} \chi\left(Q_{n, j}\right) \stackrel{*}{\rightarrow} \eta_{F}, \quad n \rightarrow \infty
$$

где

$$
3 V_{*}^{\eta_{F}}(y)+G_{E}^{\eta_{F}}(y)+3 g_{E}(y, \infty) \equiv \text { const }, \quad y \in F
$$

2) рациональная функиия $r_{n}:=Q_{n, 1} / Q_{n, 2}$ интерполирует функиию $f_{2}(z)$ (по крайней мере) в $2 n-m$ различных точках $x_{n, j}$ открытого множества 
$E^{\circ}:=\bigsqcup_{j=1}^{q}\left(e_{2 j-1}, e_{2 j}\right)$, где $m \in \mathbb{N}$ фиксировано и не зависит от $n ;$ существует предельное распределение свободных точек интерполячии $x_{n, j}$, которое совпадает с (единственной) единичной мерой $\eta_{E} \in M_{1}(E)$, имеющей носитель на $E$ и равновесной относителъно смешанного гриново-логарифмического потенииала $3 V_{*}^{\nu}(z)+G_{F}^{\nu}(z)$ :

$$
\frac{1}{2 n} \sum_{j=1}^{2 n-m} \delta_{x_{n, j}} \stackrel{*}{\rightarrow} \eta_{E}, \quad n \rightarrow \infty
$$

əəe

$$
3 V_{*}^{\eta_{E}}(x)+G_{F}^{\eta_{E}}(x) \equiv \text { const, } \quad x \in E ;
$$

3) в области $D_{2}$ справедливо следующее соотношение (ср. [93; теорема 1], [76; теорема 1.6]):

$$
\frac{Q_{n, 1}}{Q_{n, 2}} \stackrel{\text { cap }}{\longrightarrow} f_{2}(z), \quad z \in D_{2}, \quad n \rightarrow \infty
$$

скорость сходимости в (52) характеризуется соотношениями (ср. (28), (32))

$$
\begin{aligned}
\left|f_{2}(z)-\frac{Q_{n, 1}}{Q_{n, 2}}(z)\right|^{1 / n} \stackrel{\text { cap }}{\longrightarrow} e^{-2 G_{F}^{\eta_{E}}(z)}<1, \quad z \in D_{2} \backslash E, \quad n \rightarrow \infty, \\
\varlimsup_{n \rightarrow \infty}\left|f_{2}(x)-\frac{Q_{n, 1}}{Q_{n, 2}}(x)\right|^{1 / n} \leqslant e^{-2 G_{F}^{\eta_{E}}(x)}<1, \quad x \in E^{\circ},
\end{aligned}
$$

где мера $\eta_{E}$ - решение задачи (51); при этом верхняя регуляризачия функиии, стоящей в левой части неравенства (54), равна его правой части при всех $x \in E^{\circ}$.

Таким образом, в теореме 1 показано, что рациональная функция $r_{n}:=$ $Q_{n, 1} / Q_{n, 2}$, построенная по $3 n+1$ коэффициенту Лорана (в бесконечно удаленной точке) исходной функции $f_{1}=f$ - решения дифференциального уравнения $(2),-$ является многоточечной рациональной интерполяцией (в $2 n-m$ точках) функции $f_{2}$ (см. (46)), которая также является решением дифференциального уравнения (2). Подчеркнем, что у построенной рациональной функции $r_{n}$ порядка $n$ априори свободны не только нули и полюсы, но и все точки интерполяции (их число асимптотически равно $2 n$ ). При этом как точки интерполяции, так и нули и полюсы функции $r_{n}$ ведут себя "оптимальным" образом. А именно, их предельное распределение соответствует решениям экстремальных задач (97) и (121). Такие рациональные функции оказываются по своим свойствам вполне близкими к наилучшим чебышёвским рациональным аппроксимациям (см. [40], [92]), но, в отличие от последних, строятся по конечному числу коэффициентов Лорана. Рациональная функция $r_{n}$, построенная по функции $f \in \mathscr{H}(D)$, заданной дифференциальным уравнением (2), аппроксимирует в области $G \neq D$ другую ветвъ $\widetilde{f} \in \mathscr{H}(G)$ этой многозначной аналитической функции. Фактически $r_{n}$ аппроксимирует заданную многозначную аналитическую функцию $f$ на другом листе соответствующей римановой поверхности. 
ЗАмЕчаниЕ 3. В работе [76] (см. также [75]) изучен случай, когда $q=1$ и $f(z)=((z-1) /(z+1))^{\alpha}, f(\infty)=1$, где $2 \alpha \in \mathbb{C} \backslash \mathbb{Z}$. При условии, что $2 \alpha \in \mathbb{R} \backslash \mathbb{Z}$, в [76] доказан аналог теоремы 1 . Задача о распределении нулей полиномов $Q_{n, j}$ решена в [76] для указанной функции $f$ при $2 \alpha \in \mathbb{C} \backslash \mathbb{Z}$. При этом в [76] найдены явные представления для равновесных мер $\eta_{E}$ и $\eta_{F}$ :

$$
\begin{array}{ll}
\frac{d \eta_{F}}{d x}(x)=\frac{\sqrt{3}}{2 \pi} \frac{1}{\sqrt[3]{x^{2}-1}}\left(\frac{1}{\sqrt[3]{|x|-1}}-\frac{1}{\sqrt[3]{|x|+1}}\right), & x \in \overline{\mathbb{R}} \backslash[-1,1], \\
\frac{d \eta_{E}}{d x}(x)=\frac{\sqrt{3}}{4 \pi} \frac{1}{\sqrt[3]{1-x^{2}}}\left(\frac{1}{\sqrt[3]{1-x}}+\frac{1}{\sqrt{1+x}}\right), & x \in(-1,1) .
\end{array}
$$

При $q=1$ и $\alpha=1 / 3$, т. е. для функции $f(z)=((z-1) /(z+1))^{1 / 3}$, соотношение (54) в теореме 1 может быть в существенной степени уточнено. А именно, рациональная функция $Q_{n, 1} / Q_{n, 2}$ обладает свойством “почти чебышевского альтернанса" на интервале $(-1,1)$ в следующем смысле. Для любого положительного $\theta$, сколь угодно близкого к нулю, на интервале $(-1,1)$ найдется не менее $N_{n}=[2 n(1-\theta)]$ последовательных точек $x_{j},-1<x_{1}<\cdots<x_{N_{n}}$, в которых разность, стоящая под знаком модуля в левой части (54), принимает с чередующимися знаками экстремальные значения:

$$
f_{2}\left(x_{j}\right)-\frac{Q_{n, 1}}{Q_{n, 2}}\left(x_{j}\right)=(-1)^{j} \frac{2}{3} \sqrt[3]{\frac{1+x_{j}}{1-x_{j}}} e^{-2 n G_{F}^{\eta_{E}}\left(x_{j}\right)}\left(1+\varepsilon_{n}\left(x_{j}\right)\right),
$$

где $\varepsilon_{n}(x) \rightarrow 0$ при $n \rightarrow \infty$ со скоростью геометрической прогрессии локально равномерно на $(-1,1)$; под $\sqrt[3]{\cdot}$ в $(55)$ понимается арифметическое значение корня: $\sqrt[3]{a^{3}}=a$ при $a>0$.

ЗАмЕчАниЕ 4. Задача о распределении нулей полиномов Эрмита-Паде $Q_{n, j}$ в теореме 1 решена в терминах скалярной задачи равновесия (49). Возможность применения этой задачи равновесия для характеризации предельного распределения нулей основана на соотношениях ортогональности (63) (см. также (113)). Все дальнейшие рассуждения настоящей работы основаны именно на соотношениях (63). Эти же соотношения ортогональности вполне могут быть использованы и в рамках классической схемы, предложенной впервые в работе А. А. Гончара и Е. А. Рахманова [41] и основанной на том, что для вещественного случая из соотношений (63) вытекают (ср. [76]) интерполяционные условия, которым удовлетворяет функция $Q_{n, 1} / Q_{n, 2}$. Соответствующая векторная задача равновесия определяется $(2 \times 2)$-матрицей $\left(\begin{array}{cc}4 & -1 \\ -1 & 1\end{array}\right)$ и, как легко видеть, эквивалентна паре задач (49), (51). Однако мы предпочли здесь продемонстрировать новый подход к решению задачи о предельном распределении нулей, предложенный впервые Е. А. Рахмановым и С. П. Суетиным в [94] (см. также [115]). Этот подход в конечном итоге также опирается на общий GRS-метод. Однако при таком подходе интерполяционное свойство для решения задачи о предельном распределении нулей оказывается ненужным. Тем самым, этот подход расширяет область применимости GRS-метода, позволяя 
использовать его в комплексном случае, т. е. в том случае, где этого интерполяционного свойства априори нет (см. [94], [119], а также замечание 7).Установить такое интерполяционное свойство будет весьма непросто, например, уже при небольшом сдвиге хотя бы одной из точек ветвления $e_{j}$ с вещественной прямой в комплексную плоскость. Отметим, что в оригинальной работе Е. М. Никишина [78] для набора $\left[1, f_{1}, \ldots, f_{m-1}\right]$, где $m \in \mathbb{N}$ произвольно, а функции $f_{1}, \ldots, f_{m-1}$ образуют систему Никишина, решена задача об асимптотическом поведении функции $\left|R_{n}(z)\right|^{1 / n}$ при $n \rightarrow \infty$. Задача о предельном распределении нулей полиномов $Q_{n, j}$ в [78] не рассматривалась (см. также [101]-[105]).

ЗАмЕчАНИЕ 5. Пусть по-прежнему все точки ветвления $e_{j}$ вещественны, $e_{j} \in \mathbb{R}$, и различны между собой, но в представлении (41) вместо одного параметра $\alpha$ допускаются, вообще говоря, различные параметры $\alpha_{j}, 2 \alpha_{j} \in \mathbb{R} \backslash \mathbb{Z}$. Тем самым, допускается к рассмотрению более широкий, чем (41), класс функций, заданных представлением вида

$$
f(z)=\prod_{j=1}^{q}\left(\frac{z-e_{2 j-1}}{z-e_{2 j}}\right)^{\alpha_{j}}
$$

при оговоренных выше условиях на $e_{j}$ и $\alpha_{j}$. Пусть, кроме того, ветвь функции $f$ зафиксирована прежним условием: $f(\infty)=1$. В этом более широком классе для каждой функции $f, f(\infty)=1$, аналогичным образом определяется семейство функций $\tilde{f}_{j}(x):=f^{+}(x)+f^{-}(x), x \in\left(e_{2 j-1}, e_{2 j}\right)$, при этом по-прежнему каждая из этих функций является аналитическим продолжением (в смысле решения дифференциального уравнения (2)) исходной функции $f$. Однако если при некотором $j \in\{1, \ldots, q-1\}$ имеем $\alpha_{j} \neq \alpha_{j+1}$, то две соседние функции $\widetilde{f}_{j}$ и $\widetilde{f}_{j+1}$ уже не продолжаются друг в друга непосредственно ни через верхнюю полуплоскость, ни через нижнюю. Соответственно этому области голоморфности двух аналитических элементов $\left(\widetilde{f}_{j}, E_{j}^{\circ}\right)$ и $\left(\tilde{f}_{j+1}, E_{j+1}^{\circ}\right)$ не совпадают и должны быть разделены “мембраной" (ср. [11], [12], [119]). Из общих теоретических наблюдений (см. [81], [112]), подтвержденных численными экспериментами (см. [51], а также рис. 4), вытекает, что в этом случае предельное распределение нулей полиномов Эрмита-Паде характеризуется равновесной мерой, носитель которой расположен на объединении всех лакун $\left(e_{2 j}, e_{2 j+1}\right)$ и всех таких мембран, т. е. этот носитель расположен уже не на вещественной прямой, а в комплексной плоскости (разумеется, симметрично относительно вещественной прямой, что следует непосредственно из условия $f(\infty)=1$ и того факта, что все точки ветвления $e_{j}$ и параметры $\alpha_{k}$ вещественны).

Сложность общей задачи о предельном распределении нулей полиномов Эрмита-Паде видна уже на таком простом случае для набора из трех функций $\left[1, f, f^{2}\right]$. Даже в этом простом случае, когда функция $f$ задана явным представлением (56), в котором предполагается, что $e_{1}<\cdots<e_{2 q}$, но, вообще говоря, $\alpha_{j} \neq \alpha_{k}$ при $j \neq k$, предельное распределение нулей полиномов Паде $Q_{n, j}$ 1-го рода пока не удается охарактеризовать в терминах соответствующей теоретико-потенциальной задачи равновесия. В частности, не удается описать структуру и геометрические свойства возникающих мембран. Иначе 


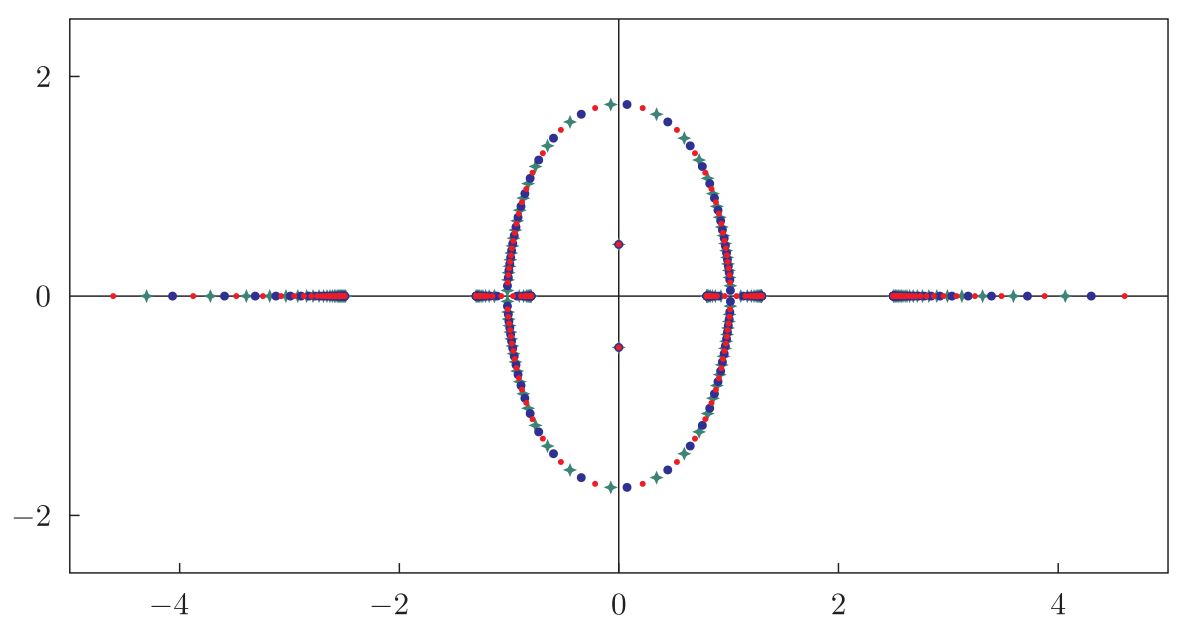

Рис. 4. Нули полиномов Эрмита-Паде $Q_{200,0}$ (синие точки), $Q_{200,1}$ (красные точки), $Q_{200,2}$ (крестики) для набора функций $\left[1, f, f^{2}\right]$, где $f(z)=\left(\frac{z+2.5}{z+1.3}\right)^{1 / 3}\left(\frac{z+0.8}{z-0.8}\right)^{-1 / 3}\left(\frac{z-1.3}{z-2.5}\right)^{1 / 3}$. Нули полиномов $Q_{200, j}$ образуют мембрану, соединяющую отрезки $[-1.3,-0.8]$ и $[0.8,1.3]$. Двум точкам пересечения мембраны с отрезками соответствуют две точки Чеботарёва. На рисунке присутствуют два триплета Фруассара (cp. [13], [50], [51]).

говоря, к настоящему моменту в теории полиномов Эрмита-Паде нет достаточно общего результата, в рамках которого можно было бы решить поставленную здесь задачу для функции вида (56).

В отличие от общего случая произвольных $\alpha_{j}, 2 \alpha_{j} \in \mathbb{R} \backslash \mathbb{Z}$, в том частном случае, когда все $\alpha_{j}$ равны $\alpha$ и $2 \alpha \in \mathbb{R} \backslash \mathbb{Z}$, как уже было отмечено выше, все ростки $\left(\tilde{f}_{j}, E_{j}^{\circ}\right)$ являются "следами" (каждый на своем интервале $E_{j}^{\circ}$ ) одной и той же ветви многозначной аналитической функции $\tilde{f}$ (решения дифференциального уравнения (2)), голоморфной в области $G=\overline{\mathbb{C}} \backslash F$, т. е. в комплексной плоскости с конечным множеством разрезов, проведенных по отрезкам вещественной прямой.

Таким образом, уже в случае различных вещественных параметров $\alpha_{j}, 2 \alpha_{j} \in$ $\mathbb{R} \backslash \mathbb{Z}$, при сохранении в представлении (41) того же условия $e_{1}<\cdots<e_{2 q}$ на точки ветвления теоретико-потенциальная задача на равновесную меру $\eta_{E}$ с носителем на $E$, соответствующая задаче о распределении нулей полиномов Эрмита-Паде, становится комплексной в следующем смысле. Эта задача по-прежнему имеет вид (49). Однако соответствующий компакт $F$ уже, вообще говоря, не совпадает с множеством $\overline{\mathbb{C}} \backslash E$. В такой более общей постановке этот компакт $F$ заранее не известен - он сам становится неизвестным параметром задачи. Из общих результатов, связанных с асимптотическим поведением неэрмитово ортогональных многочленов (см. прежде всего [44], [110], [111], а также [8]), известно, что этот априори неизвестный компакт $F$ обязательно должен обладать определенным характеристическим свойством “симметрии”, есте- 
ственным образом связанным с рассматриваемым классом потенциалов и семейством допустимых компактов. Иначе говоря, этот компакт $F$ является $S$-кривой, ассоциированной с заданным классом потенциалов (об этом понятии см. [60], [91]). Доказательство существования такой $S$-кривой и описание ее характеристических свойств, как правило, представляет собой весьма трудную задачу, к настоящему времени решенную только в некоторых случаях, а именно:

1) для логарифмического потенциала $V^{\mu}(z)$ и произвольной многозначной аналитической функции с конечным множеством точек ветвления (классические АП, Г. Шталь [107]-[109]; см. п. 2.2);

2 ) для логарифмического потенциала $V^{\mu}(z)$ с внешним полем $V_{*}^{-\nu}(z)$, заданным потенциалом отрицательного единичного заряда, сосредоточенного в конечном множестве точек $\left\{z_{1}, \ldots, z_{m}\right\}$ комплексной плоскости, и произвольного мультиростка с конечным множеством точек ветвления (m-точечные АП; В. И. Буслаев [21]-[23]; см. п. 2.3);

$3)$ для смешанного гриново-логарифмического потенциала $3 V^{\mu}(z)+G_{E}^{\mu}(z)$ с внешним полем $\psi(z)=3 g_{E}(z, \infty)$, где $E$ - объединение конечного множества отрезков, расположенных на вещественной прямой, $\mu \in M_{1}(K), K \in \mathfrak{K}_{f}^{(3)}, \mathfrak{K}_{f}^{(3)}$ соответствующее семейство допустимых компактов; Е. А. Рахманов и С. П. Суетин [93], [94]; см. также [23], [72].

Все эти три задачи удалось решить с помощью вариационного метода, предложенного в [88], а именно, метода, основанного на вариации функционала энергии, соответствующего исследуемой задаче о предельном распределении нулей полиномов Паде (ниже $\mathfrak{K}_{f}^{(1)}, \mathfrak{K}_{f}^{(2)}, \mathfrak{K}_{f}^{(3)}$ - различные классы допустимых компактов для многозначной функции $f$ ).

В классическом случае имеем:

$$
\inf _{K \in \mathfrak{K}_{f}^{(1)}} \operatorname{cap} K=\exp \left\{-\sup _{K \in \mathfrak{K}_{f}^{(1)}} J\left(K ; \lambda_{K}\right)\right\},
$$

где $\lambda_{K}-$ равновесная мера для $K \in \mathfrak{K}_{f}^{(1)}$ и

$$
J(K ; \mu)=\iint \log \frac{1}{|z-\zeta|} d \mu(\zeta) d \mu(z)
$$

- соответствующий функционал энергии, $\mu \in M_{1}(K)$.

В случае, соответствующем многоточечным АП, для функционала энергии с внешним полем имеем:

$$
\begin{aligned}
I_{\psi}(K ; \mu) & =\iint\left(\log \frac{1}{|z-\zeta|}+\psi(z)+\psi(\zeta)\right) d \mu(z) d \mu(\zeta) \\
& =\int_{K}\left(V^{\mu}(z)+2 \psi(z)\right) d \mu(z),
\end{aligned}
$$

$\psi(z)=V_{*}^{-\nu}(z)$ - внешнее поле, порожденное отрицательным единичным зарядом $-\nu, \nu=\sum_{j=1}^{m} p_{j} \delta_{z_{j}}$, сосредоточенным в точках интерполяции $z_{1}, \ldots, z_{m}$, $K \in \mathfrak{K}_{f}^{(2)}$. 
И, наконец, в случае аппроксимаций Эрмита-Паде для набора $\left[1, f_{1}, f_{2}\right]$, где пара функций $f_{1}, f_{2}$ образует обобщенную систему Никишина, соответствующий функционал энергии имеет вид

$$
\begin{aligned}
J_{3}(K ; \mu) & =\iint\left(3 \log \frac{1}{|x-y|}+g_{K}(x, y)\right) d \mu(x) d \mu(y) \\
& =\int_{E}\left(3 V^{\mu}(x)+G_{K}^{\mu}(x)\right) d \mu(x)
\end{aligned}
$$

и рассматривается в классе всех мер $\mu \in M_{1}(E)$, где $E$ - объединение конечного множества отрезков, расположенных на вещественной прямой, $g_{K}(x, y)-$ функция Грина для допустимого компакта $K \in \mathfrak{K}_{f}^{(3)}$.

Соответственно, в каждом из трех случаев существует (и единствен) свой допустимый $S$-компакт $F \in \mathfrak{K}_{f}^{(j)}, j \in\{1,2,3\}$, который вполне характеризуется следующим $S$-свойством (или свойством симметрии):

1) в первом случае $F=S \in \mathfrak{K}_{f}^{(1)}-$ компакт Шталя, т. е.

$$
\frac{\partial g_{D}(\zeta, \infty)}{\partial n^{+}}=\frac{\partial g_{D}(\zeta, \infty)}{\partial n^{-}}, \quad \zeta \in S^{\circ}
$$

где $S^{\circ}$ - совокупность открытых аналитических дуг, составляющих $S, \partial / \partial n^{ \pm}-$ производные по нормали с противоположных сторон $S^{\circ}$ (этот результат принадлежит Шталю, но он также доказывается по схеме, предложенной в [88]);

$2)$ во втором случае $F \in \mathfrak{K}_{f}^{(2)}$ обладает $S$-свойством, если

$$
\frac{\partial\left(V^{\beta}+\psi\right)}{\partial n^{+}}(\zeta)=\frac{\partial\left(V^{\beta}+\psi\right)}{\partial n^{-}}(\zeta), \quad \zeta \in F^{\circ},
$$

где $\beta \in M_{1}(F)$ - соответствующая равновесная мера, $\psi(z)=V_{*}^{-\nu}(z)-$ внешнее поле, $F^{\circ}$ - объединение всех открытых дуг, принадлежащих компакту $F$, $\partial / \partial n^{ \pm}$- нормальные производные, взятые с противоположных сторон $F^{\circ}$ (В. И. Буслаев [21]);

3) наконец, в третьем случае $F=F(3) \in \mathfrak{K}_{f}^{(3)}$ обладает $S$-свойством (или является $S$-кривой), если

$$
\frac{\partial G_{F}^{\eta_{E}}}{\partial n^{+}}(\zeta)=\frac{\partial G_{F}^{\eta_{E}}}{\partial n^{-}}(\zeta), \quad \zeta \in F^{\circ},
$$

где $\eta_{E} \in M_{1}(E)$ - соответствующая равновесная мера, $F^{\circ}$ - объединение всех открытых дуг, принадлежащих компакту $F, \partial / \partial n^{ \pm}$- нормальные производные, взятые с противоположных сторон $F^{\circ}$ (этот результат получен в [73], $G_{F}^{\mu}-$ гринов потенциал).

Соотношения (60)-(62) внешне выглядят по-разному. Однако все они определяют $S$-кривые, соответствующие вполне конкретным задачам.

\section{3. Доказательство теоремы 1}

Теорема 1 доказывается GRS-методом. В настоящей работе для краткости изложения мы приводим только схему доказательства и только для случая, когда выполняется соотношение (101). Общий случай сводится к этому част- 
ному случаю с помощью аналога леммы 5 из [94] (ср. [44; лемма 9]). Отметим, что основы GRS-метода были заложены в 1981-1984 гг. в работах A. А. Гончара и Е.А. Рахманова [41] и [42], где впервые исследовалась задача о предельном распределении нулей многочленов, ортогональных относительно переменного (т. е. зависящего от номера многочлена) веса на вещественной прямой. Эта задача была решена в терминах логарифмических потенциалов с внешними полями (см. также монографию [96] 1997 г.). В 1985-1986 гг. Г. Шталь [107]-[111] в связи с задачей о сходимости АП для многозначных аналитических функций исследовал задачу о предельном распределении нулей полиномов, ортогональных на $S$-кривых, но без переменного веса. В 1987 г. А. А. Гончар и Е. А. Paxманов [44] в связи с решением “гипотезы об 1/9" (см. [124], а также Wolfram MathWorld: “One-ninth constant”, 14) впервые сформулировали и исследовали задачу о распределении полиномов, ортогональных на взвешенных $S$-кривых.

В полном соответствии с GRS-методом доказательство теоремы 1 основано на соотношениях ортогональности, которые выполняются для полиномов Эрмита-Паде $Q_{n, 2}$, а именно на соотношениях (94) (см. также [94], ср. [66]). Это - типичные соотношения ортогональности с переменным весом (см. [41], [40], [42], [58], [94]). Однако, поскольку переменный вес $\Psi_{n}$ сам зависит от (произвольного) полинома $\omega_{n}$ (см. (93)), результаты теоремы 1 не могут быть получены напрямую из общих теорем работы [42]. Более того, в [42] предельное распределение нулей ортогональных полиномов характеризуется в терминах равновесных мер для логарифмических потенциалов с внешними полями. В настоящей работе доказано, что предельное распределение нулей полиномов Эрмита-Паде совпадает с равновесной мерой для смешанного (гриново-логарифмического) потенциала с внешним полем.

3.1. Доказательство утверждения 1) теоремы 1. Пусть функция $f$ задана представлением (41), где $\alpha \in(-1 / 2,1 / 2), \alpha \neq 0$, и пусть условием $f(\infty)=1$ зафиксирована ветвь этой функции в точке $z=\infty$. Пусть $Q_{n, j} \in$ $\mathbb{R}_{n}[z], Q_{n, j} \not \equiv 0,-$ соответствующие полиномы Эрмита-Паде для мультииндекса $(n, n, n)$, т. е. выполняется соотношение (42). Непосредственно из этого соотношения вытекает, что для произвольного полинома $q \in \mathbb{P}_{2 n}$ выполняется равенство (ср. (12))

$$
\int_{\Gamma}\left(Q_{n, 1} f+Q_{n, 2} f^{2}\right)(z) q(z) d z=0
$$

где $\Gamma$ - произвольный замкнутый контур, который отделяет множество $E$ от бесконечно удаленной точки $z=\infty$. Пусть $P_{n, 0}, P_{n, 1}-$ полиномы Паде для функции $f$ в бесконечно удаленной точке, т. е. $P_{n, 0}, P_{n, 1} \in \mathbb{R}_{n}[z], P_{n, j} \not \equiv 0$, и выполнено соотношение

$$
H_{n}(z):=\left(P_{n, 0}+P_{n, 1} f\right)(z)=O\left(\frac{1}{z^{n+1}}\right), \quad z \rightarrow \infty
$$

\footnotetext{
${ }^{14}$ Соответствующая постоянная известна как постоянная Альфана [44], [48], а также как постоянная Варги, см. Wolfram MathWorld: "Varga's constant".
} 
$H_{n}$ - функция остатка. Из (64) вытекает, что для произвольного полинома $p \in \mathbb{P}_{n-1}$ выполняется соотношение

$$
\int_{\Gamma} P_{n, 1}(\zeta) f(\zeta) p(\zeta) d \zeta=0
$$

Положим теперь в (63)

$$
q=P_{n+k, 1}, \quad k=1, \ldots, n .
$$

Тогда в силу (65) из (63) получаем соотношение

$$
\int_{\Gamma}\left(Q_{n, 2} f^{2}\right)(\zeta) P_{n+k}(\zeta) d \zeta=0, \quad k=1, \ldots, n,
$$

где мы обозначили $P_{n+k, 1}$ через $P_{n+k}$; мы будем использовать это обозначение всюду в дальнейшем. Положим

$$
\widetilde{f}(x)=\left(f^{+}+f^{-}\right)(x), \quad x \in E^{\circ} .
$$

Поскольку $|\alpha| \in(0,1 / 2)$, функция $\Delta\left(f^{2}\right)(x)=\widetilde{f}(x) \Delta f(x)$ интегрируема на $E$. Следовательно, соотношения (67) можно эквивалентным образом переписать в виде

$$
\int_{E} Q_{n, 2}(x) \widetilde{f}(x) P_{n+k}(x) \Delta f(x) d x=0, \quad k=1, \ldots, n .
$$

Поскольку $\alpha \in \mathbb{R}$, из представления (41) вытекает, что const $\widetilde{f}(x) \Delta f(x)>0$ при $x \in E^{\circ}$, где const $\neq 0$. Ниже будет показано, что из соотношений ортогональности (69) следует, что все нули полиномов $Q_{n, 2}$, за возможным исключением конечного их числа, лежат на $F^{\circ}$.

Пусть теперь $\gamma=\bigsqcup_{j=1}^{q} \gamma_{j}$ - объединение $q$ попарно непересекающихся замкнутых аналитических контуров $\gamma_{j}, \gamma_{j} \cap \gamma_{k}=\varnothing, j \neq k$, каждый из которых устроен следующим образом. Контур $\gamma_{j}$ симметричен относительно вещественной прямой, проходит через точки $e_{2 j-1}$ и $e_{2 j}$ и содержит внутри себя открытый интервал $\left(e_{2 j-1}, e_{2 j}\right)$. Будем считать все контуры $\gamma_{j}$ ориентированными по часовой стрелке. Поскольку в силу представления (41) все функции $\widetilde{f}_{j}(x):=\left(f^{+}+f^{-}\right)(x), x \in E_{j}^{\circ}, j=1, \ldots, q$, являются следами одной и той же функции $\tilde{f}$, голоморфной (аналитической и однозначной) в области $G=\overline{\mathbb{C}} \backslash F$, то каждая из функций $\widetilde{f}_{j}$ голоморфна внутри области $G_{j}:=\operatorname{int} \gamma_{j} \supset E_{j}^{\circ}$ и интегрируема на $\gamma_{j}, \widetilde{f}_{j}=\left.\widetilde{f}\right|_{G_{j}}$. В силу (64) скачок $\Delta H_{n}$ функции остатка $H_{n}$ на $E^{\circ}$ равен $\Delta H_{n}(x)=P_{n}(x) \Delta f(x), x \in E^{\circ}$. Тем самым, $\Delta H_{n}$ - интегрируемая на $E$ функция. Поскольку $|\alpha| \in(0,1 / 2)$, то $H_{n}$ - также интегрируемая функция на $\gamma=\bigsqcup_{j=1}^{q} \gamma_{j}$. В теории ортогональных полиномов $H_{n}$ называют функцией 2-го рода, соответствующей ортогональным полиномам $P_{n}$; в дальнейшем мы будем использовать именно этот термин, чтобы избежать путаницы с функцией остатка $R_{n}$ (см. (42)). Непосредственно из (64) вытекает, что для функции 2-го рода $H_{n}$ справедливо следующее представление:

$$
H_{n}(z)=\frac{1}{p(z)} \frac{1}{2 \pi i} \int_{\Gamma} \frac{P_{n}(\zeta) p(\zeta)}{\zeta-z} f(\zeta) d \zeta
$$


где $z \in \operatorname{ext} \Gamma$ (т. е. точка $z$ лежит в той связной компоненте дополнения к Г, которая содержит бесконечно удаленную точку), контур Г ориентирован по часовой стрелке и содержит внутри себя $\gamma$; а $p \in \mathbb{P}_{n}-$ произвольный полином степени $\leqslant n$. Непосредственно из $(64)$ втекают следующие свойства функции 2-го рода: функция 2-го рода $H_{n}$ имеет в бесконечно удаленной точке нуль порядка $n+1$, она голоморфна вне $E$ и имеет интегрируемый скачок $\Delta H_{n}$ на $E, \Delta H_{n}(x)=P_{n}(x) \Delta f(x)$ при $x \in E^{\circ}$, каждая из $q$ функций $\Delta H_{n}(x), x \in$ $\left(e_{2 j-1}, e_{2 j}\right)$, голоморфно продолжается с интервала $E_{j}^{\circ}$ в область $G_{j}$ и является следом в этой области $G_{j}$ одной и той же функции $P_{n} \widehat{f}$, голоморфной в области $G \supset G_{j}, G=\overline{\mathbb{C}} \backslash F, j=1, \ldots, q ;\left.\widehat{f}\right|_{E_{j}^{\circ}}=\Delta f(x)=f^{+}(x)-f^{-}(x), x \in E_{j}^{\circ}$.

В силу условий теоремы 1 функция $H_{n} \widetilde{f}_{j}$ интегрируема на $\gamma_{j}$. Следовательно, определены интегралы

$$
\int_{\gamma_{j}} Q_{n, 2}(\zeta) H_{n+k}(\zeta) \tilde{f}_{j}(\zeta) d \zeta, \quad \tilde{f}_{j} \in \mathscr{H}\left(G_{j}\right), \quad \gamma_{j}=\partial G_{j}, \quad j=1, \ldots, q,
$$

а тем самым, и соответствующий интеграл по $\gamma=\bigsqcup_{j=1}^{q} \gamma_{j}$ :

$$
\int_{\gamma} Q_{n, 2}(\zeta) H_{n+k}(\zeta) \widetilde{f}(\zeta) d \zeta, \quad k=1,2, \ldots
$$

Теперь уже нетрудно увидеть, что справедливо равенство

$$
\int_{\gamma} Q_{n, 2}(\zeta) H_{n+k}(\zeta) \tilde{f}(\zeta) d \zeta=\int_{E} Q_{n, 2}(x) P_{n+k}(x) \tilde{f}(x) \Delta f(x) d x .
$$

В силу (69) последний интеграл равен нулю при $k=1, \ldots, n$. Следовательно, имеют место равенства

$$
\int_{\gamma} Q_{n, 2}(\zeta) H_{n+k}(\zeta) \widetilde{f}(\zeta) d \zeta=0, \quad k=1, \ldots, n,
$$

где $\left.\widetilde{f}\right|_{G_{j}}=\widetilde{f}_{j}$. Поскольку функция $H_{n+k}$ имеет в бесконечно удаленной точке нуль порядка $n+k+1 \geqslant n+2, Q_{n, 2} \in \mathbb{P}_{n}$ и функция $\tilde{f}$ ограничена в окрестности бесконечно удаленной точки, то из соотношений (72) вытекают равенства

$$
\int_{F} Q_{n, 2}(y) H_{n+k}(y) \Delta \widetilde{f}(y) d y=0, \quad k=1, \ldots, n
$$

(в силу вышеперечисленных условий все интегралы в (73) существуют). Совокупность равенств (73) эквивалентным образом переписывается в следующем виде:

$$
\int_{F} Q_{n, 2}(y)\left(\sum_{k=1}^{n} c_{k} H_{n+k}(y)\right) \Delta \tilde{f}(y) d y=0,
$$

где $c_{k}, k=1, \ldots, n,-$ произвольные комплексные числа. Воспользуемся теперь тем хорошо известным фактом [120], что функции 2-го рода $H_{n}$, так же 
как и монические ортогональные полиномы $P_{n}(z)=z^{n}+\cdots$, удовлетворяют следующим трехчленным рекуррентным соотношениям (ср. (13)):

$$
H_{n}(z)=\left(z-\widehat{b}_{n}\right) H_{n-1}(z)-\widehat{a}_{n}^{2} H_{n-2}(z), \quad n=1,2, \ldots,
$$

где $H_{-1}(z) \equiv 1, H_{0}(z) \equiv f(z)$; при этом в силу заданных условий на функцию $f$ все $\widehat{a}_{n}^{2}$ положительны. Предположим, что $n=2 m$ - четное число; случай нечетного $n$ рассматривается аналогично. Тогда с помощью рекуррентных соотношений (75) при произвольных комплексных постоянных $c_{1}, \ldots, c_{n}$ получаем тождество

$$
\sum_{k=1}^{n} c_{k} H_{n+k}(z)=q_{n, 1}(z) H_{n+m}(z)+q_{n, 2}(z) H_{n+m+1}(z),
$$

где полиномы $q_{n, 1}, q_{n, 2} \in \mathbb{P}_{m-1}$ произвольны, поскольку произвольны постоянные $c_{1}, \ldots, c_{n}$. Таким образом, соотношение (74) эквивалентным образом переписывается в виде

$$
\int_{F} Q_{n, 2}(y)\left\{q_{n, 1}(y) H_{n+m}(y)+q_{n, 2}(y) H_{n+m+1}(y)\right\} \Delta \widetilde{f}(y) d y=0 .
$$

Подчеркнем, что, поскольку в (74) коэффициенты $c_{1}, \ldots, c_{n}$ могут быть выбраны произвольно, полиномы $q_{n, 1}, q_{n, 2} \in \mathbb{P}_{m-1}$ в соотношении (77) также могут быть выбраны произвольно. Перепишем теперь соотношение (77) в виде

$$
\int_{F} Q_{n, 2}(y)\left\{q_{n, 2}(y) \frac{H_{n+m+1}(y)}{H_{n+m}(y)}-q_{n, 1}(y)\right\} H_{n+m}(y) \Delta \widetilde{f}(y) d y=0
$$

(при переходе от $(77)$ к (78) мы поменяли знак у произволъного полинома $q_{n, 1} \in$ $\left.\mathbb{P}_{m-1}\right)$.

Воспользуемся теперь тем, что функция $f$, заданная формулой (41), представляется в виде $f(z)=1+$ const $\widehat{\sigma}(z)$, где const $\neq 0$, а $\widehat{\sigma}$ - марковская функция, соответствующая положительной мере $\sigma$ с носителем на $E$. Следовательно, для отношения двух функций остатка $H_{n+1}$ и $H_{n}$ при $n \geqslant 1$ справедливо представление (см. [120; рус. изд., добавление Я. Л. Геронимуса, гл. IV, формула (IV.5)])

$$
\frac{H_{n+1}}{H_{n}}(z)=\frac{\widehat{a}_{n+2}^{2}}{z-\widehat{b}_{n+2}-\frac{\widehat{a}_{n+3}^{2}}{z-\widehat{b}_{n+3}-\cdots}},
$$

где все $\widehat{a}_{k}, \widehat{b}_{k}$ вещественны, $\widehat{a}_{k} \neq 0$ и $\left\{\widehat{b}_{n+2}, \widehat{b}_{n+3}, \ldots\right\} \Subset \mathbb{R}$. В таком случае по теореме Фавара [120] имеем

$$
\frac{H_{n+1}}{H_{n}}(z)=\widehat{\sigma}_{n}(z)
$$

где $\sigma_{n}$ - положительная мера с носителем supp $\sigma_{n} \Subset \mathbb{R}$. Пусть $\widehat{H}_{n}(z)-$ функции 2 -го рода, соответствующие ортонормированным полиномам $\widehat{P}_{n}(z)=\widehat{P}_{n, 1}(z)$. В следующем вспомогательном утверждении уточняются свойства меры $\sigma_{n}$. 
Лемма 1. В условиях теоремы 1 отношение $H_{n+1} / H_{n}$ представляется в виде

$$
\frac{H_{n+1}}{H_{n}}(z)=\widehat{\rho}_{n}(z)+\sum_{j=1}^{q-1} \frac{c_{n, j}}{z-x_{n, j}}=\int_{E} \frac{\rho_{n}(x) d x}{z-x}+\sum_{j=1}^{q-1} \frac{c_{n, j}}{z-x_{n, j}}, \quad z \notin E,
$$

где все $c_{n, j}$ неотрицательны, точки $x_{n, j}$ принадлежат отрезку $\left[e_{2 j}, e_{2 j+1}\right] u$

$$
\rho_{n}(x)=-\frac{1}{2 \pi i} \frac{\Delta f(x)}{\widehat{H}_{n}^{+}(x) \widehat{H}_{n}^{-}(x)}, \quad x \in E^{\circ} .
$$

ДокАзАТЕЛЬство. Из рекуррентных соотношений (75), записанных в двух разных точках $z$ и $\zeta$,

$$
\begin{aligned}
& H_{n+1}(z)=\left(z-\widehat{b}_{n+1}\right) H_{n}(z)-\widehat{a}_{n+1}^{2} H_{n-1}(z), \\
& H_{n+1}(\zeta)=\left(\zeta-\widehat{b}_{n+1}\right) H_{n}(\zeta)-\widehat{a}_{n+1}^{2} H_{n-1}(\zeta),
\end{aligned}
$$

получаем

$$
\begin{gathered}
H_{n+1}(z) H_{n}(\zeta)-H_{n+1}(\zeta) H_{n}(z)=(z-\zeta) H_{n}(z) H_{n}(\zeta) \\
-\widehat{a}_{n+1}^{2}\left\{H_{n}(\zeta) H_{n-1}(z)-H_{n}(z) H_{n-1}(\zeta)\right\} .
\end{gathered}
$$

Непосредственно из (82) вытекает, что для $x \in E^{\circ}$ соответствующие предельные значения функций 2-го рода связаны соотношением

$$
\begin{gathered}
H_{n+1}^{+}(x) H_{n}^{-}(x)-H_{n+1}^{-}(x) H_{n}^{+}(x)=\prod_{k=1}^{n+1} \widehat{a}_{k}^{2} \cdot \Delta H_{0}(x) \\
=\prod_{k=1}^{n+1} \widehat{a}_{k}^{2} \cdot \Delta f(x)=\frac{\Delta f(x)}{k_{n}^{2}}, \quad x \in E^{\circ} ;
\end{gathered}
$$

здесь $k_{n}>0$ - старший коэффициент соответствующего полинома $\widehat{P}_{n}(x)=$ $k_{n} x^{n}+\cdots=k_{n} P_{n}(x)$, ортонормированного относительно положительной меры $d \sigma(x)=$ const $\Delta f(x) d x, x \in E$. Непосредственно из (83) получаем, что

$$
\frac{H_{n+1}^{+}}{H_{n}^{+}}(x)-\frac{H_{n+1}^{-}}{H_{n}^{-}}(x)=\frac{\Delta f(x)}{\widehat{H}_{n}^{+}(x) \widehat{H}_{n}^{-}(x)} \sigma_{n}^{\prime}(x), \quad x \in E^{\circ},
$$

где $\widehat{H}_{n}$ - функция 2-го рода, соответствующая ортонормированному полиному $\widehat{P}_{n}$. Полиномы $P_{n}$ ортогональны на $E$ относительно положительной меры $d \sigma(x)=$ const $\Delta f(x) d x$. Следовательно, все нули $P_{n}$ лежат в $\widehat{E}-$ выпуклой оболочке компакта $E$. При этом в каждой лакуне $\left(e_{2 j}, e_{2 j+1}\right), j=1, \ldots, q-1$, может лежать не более одного нуля полинома $P_{n}$. Непосредственно из формулы (70) вытекает, что соответствующая функция 2-го рода $H_{n}$ кроме нуля порядка $n+1$ в бесконечно удаленной точке может иметь не более $q-1$ дополнительных нулей (т. е. дополнительных точек интерполяции функции $f$ аппроксимацией Паде $[n / n]_{f}$ ), расположенных не более чем по одному в $q-1$ лакунах 
между отрезками $E_{j}$. Из сказанного и формулы (84) вытекает представление

$$
\frac{H_{n+1}}{H_{n}}(z)=\widehat{\rho}_{n}(z)+\sum_{j=1}^{q-1} \frac{c_{n, j}}{z-x_{n, j}}
$$

где все $c_{n, j}$ неотрицательны (поскольку $\sigma_{n}$ - мера), точки $x_{n, j}$ принадлежат $\left[e_{2 j}, e_{2 j+1}\right]$ и

$$
\rho_{n}(x)=\sigma_{n}^{\prime}(x)=-\frac{1}{2 \pi i} \frac{\Delta f(x)}{\widehat{H}_{n}^{+}(x) \widehat{H}_{n}^{-}(x)}, \quad x \in E^{\circ} .
$$

Лемма доказана.

Из результатов работы [74] следует, что для функции $f \in \mathscr{L}_{E}$

$$
\frac{\widehat{H}_{n}^{+}(x) \widehat{H}_{n}^{-}(x)}{\prod_{j=1}^{q-1}\left(x-x_{n, j}\right)} \rightrightarrows 1, \quad n \rightarrow \infty, \quad x \in K \Subset E^{\circ}
$$

Таким образом, из леммы 1 вытекает, что $d \sigma_{n}(x)=\rho_{n}(x) d x+\sum_{j=1}^{q-1} \delta_{x_{n, j}}$, и из (78)
получаем следующее соотношение:

$$
\int_{F} Q_{n, 2}(y)\left\{q_{n, 2}(y) \widehat{\rho}_{n}(y)-q_{n, 1}(y)\right\} H_{n+m}(y) \tau_{n}(y) \Delta \widetilde{f}(y) d y=0,
$$

где

$$
\tau_{n}(z):=\prod_{j=1}^{q-1}\left(z-x_{n, j}\right)
$$

и $q_{n, 1}, q_{n, 2} \in \mathbb{P}_{m-q}$ - произвольные полиномы степени $\leqslant m-q$; при переходе от $(78)$ к (88) мы, потеряв в степени произвольных полиномов $q_{n, 1}, q_{n, 2}$, избавились от рациональной части в представлении (80).

Пусть теперь $\omega_{n} \in \mathbb{P}_{2 m-2 q+1}$ - монический полином степени $\leqslant 2 m-2 q+1$, все корни которого простые и лежат на $F^{\circ}$, и $\omega_{n}^{*}$ - соответствующий сферически нормированный полином. Подберем полиномы $q_{n, 1}, q_{n, 2} \in \mathbb{P}_{m-q}$ так, чтобы следующая функция была голоморфна на $F^{\circ}$ :

$$
\frac{q_{n, 2}(z) \widehat{\rho}_{n}(z)-q_{n, 1}(z)}{\omega_{n}^{*}(z)} ;
$$

очевидно, что это условие эквивалентно определенным интерполяционным соотношениям, выполнения которых, учитывая число свободных параметров, всегда можно добиться с помощью выбора полиномов $q_{n, 1}, q_{n, 2} \in \mathbb{P}_{m-q}$. Из (89) вытекает, что полиномы $q_{n, 2}$ ортогональны на $E$ относительно переменной меры $d \rho_{n}(x) / \omega_{n}^{*}(x)$, а именно, справедливы соотношения

$$
\int_{E} q_{n, 2}(x) x^{s} \frac{d \rho_{n}(x)}{\omega_{n}^{*}(x)}=0, \quad s=0,1, \ldots, m-q-1 .
$$


Из (90) следует, что все нули полинома $q_{n, 2}$ лежат в conv $E$, точнее, все нули этого полинома, за возможным исключением не более чем $q-1$, лежат на $E$, и справедливо представление

$$
\frac{q_{n, 2}(z) \widehat{\rho}_{n}(z)-q_{n, 1}(z)}{\omega_{n}^{*}(z)}=\frac{1}{p(z)} \int_{E} \frac{q_{n, 2}(x) p(x) d \rho_{n}(x)}{\omega_{n}^{*}(x)(z-x)}, \quad z \notin E,
$$

где $p(z) \in \mathbb{P}_{m-q}-$ произвольный полином степени $\leqslant m-q . \quad$ С учетом $(91)$ соотношения (88) переписываются в виде

$$
0=\int_{F} Q_{n, 2}^{*}(y) \frac{\omega_{n}^{*}(y)}{q_{n, 2}(y)}\left\{\int_{E} \frac{q_{n, 2}^{2}(x) d \rho_{n}(x)}{\omega_{n}^{*}(x)(y-x)}\right\} H_{n+m+1}(y) \tau_{n}(y) \Delta \widetilde{f}(y) d y .
$$

Подчеркнем, что в $(92) \omega_{n}^{*}-$ произвольный полином степени $\leqslant 2 m-2 q+1$. Положим

$$
\begin{aligned}
\Psi_{n}(z) & :=\frac{1}{q_{n, 2}(z)}\left\{\int_{E} \frac{q_{n, 2}^{2}(x) d \rho_{n}(x)}{\omega_{n}^{*}(x)(z-x)}\right\} H_{n+m+1}(z) \tau_{n}(z) \Delta \widetilde{f}(z) \\
& =\frac{1}{\widetilde{q}_{n, 2}(z)}\left\{\int_{E} \frac{q_{n, 2}(x) \widetilde{q}_{n, 2}(x) d \rho_{n}(x)}{\omega_{n}^{*}(x)(z-x)}\right\} H_{n+m+1}(z) \tau_{n}(z) \Delta \widetilde{f}(z), \quad z \notin E,
\end{aligned}
$$

где $\widetilde{q}_{n, 2} \in \mathbb{P}_{m-q}$. Тогда соотношения (92) примут вид

$$
\int_{F} Q_{n, 2}^{*}(y) \omega_{n}^{*}(y) \Psi_{n}(y) d y=0
$$

для любого полинома $\omega_{n}^{*} \in \mathbb{P}_{2 m-2 q+1}$; функция $\Psi_{n}$ играет здесь роль переменного (т. е. зависящего от номера $n$ ) веса. В силу определения (93) функция $\Psi_{n}$ в (94) сама зависит от полинома $\omega_{n}^{*}$. Нетрудно увидеть, что если в (93) полиномы $\omega_{n}^{*}$ и $q_{n, 2}$ вещественные, то функция $\Psi_{n}$ также будет вещественной и может иметь на $F$ только конечное число перемен знака, не зависящее от $n$. Отсюда уже легко вытекает, что все нули полинома $Q_{n, 2}$, за исключением некоторого, не зависящего от $n$ числа, лежат в $F$ и степень полинома $Q_{n, 2}$ может быть меньше $n$ только на некоторое число, ограниченное при $n \rightarrow \infty$.

ЗАмЕчАниЕ 6. Исследуемая в настоящей статье задача изначально обладает определенной, а именно вещественной, симметрией. Тем самым, соотношения (94) можно рассматривать как соотношения квазиортогональности с переменным, т. е. зависящим от номера полинома $Q_{n, 2}$, весом $\Psi_{n}$. Эти соотношения вполне можно использовать как исходные для применения общего метода, разработанного А. А. Гончаром и Е. А. Рахмановым в [41]-[44] в связи с решением ими "гипотезы об $1 / 9$ " и предназначенного для изучения предельного распределения нулей полиномов, (неэрмитово) ортогональных с переменным весом. Хорошо известно (см. прежде всего оригинальную работу [44], а также [32], [40]), что в основе применения этого метода лежит существование компакта, обладающего определенной “симметрией” в рамках рассматриваемой задачи, а именно существование так называемой $S$-кривой, ассоциированной 
с изучаемой задачей. В изучаемой нами задаче существование $S$-кривой (точнее, пары $S$-кривых $E$ и $F$ ) вытекает уже из исходной постановки задачи, а именно - из ее вещественной симметрии. Говоря более точно, из вещественной симметрии решаемой задачи вытекает существование пары $S$-кривых $E$ и $F$, образующих так называемый конденсатор Наттолла $(E, F)$, пластины $E$ и $F$ которого обладают свойством взаимной симметрии; по поводу этого понятия см. прежде всего работу [93], где оно было впервые введено, а также [57], [94]. Тот факт, что в $(94)$ переменный вес $\Psi_{n}$ сам зависит от полинома $\omega_{n}^{*}$, вообще говоря, не ограничивает применимости GRS-метода; см. по этому поводу [94], а также [92; гипотеза 2].

Рассмотрим теперь следующую задачу равновесия (см. [24], [93], [94]).

ЗАДАЧА РАВНОВЕСИЯ 1. Пусть $\mu \in M_{1}(F)$ - произвольная (положительная борелевская) единичная мера $\mu$ с носителем на $F$. Обозначим через $V_{*}^{\mu}(z)$, $z \notin F$, сферически нормированный логарифмический, а через

$$
G_{E}^{\mu}(z):=\int_{F} g_{E}(x, z) d \mu(x), \quad z \notin F,
$$

гринов (относительно области D) потенциаль меры $\mu$, где $g_{E}(x, z)$ - функция Грина для области D. Здесь и в дальнейшем мы предполагаем, что все используемые функиии существуют; без ограничения общности будем считать, чmo $\operatorname{conv} E=[-1,1]$.

Рассмотрим следуюшую экстремальную задачу для смешанного (гриновологарифмического) потенииала $3 V_{*}^{\mu}(z)+G_{E}^{\mu}(z)$ меры $\mu \in M_{1}(F)$ с внешним полем $\psi(z)=3 g_{E}(z, \infty)$ :

$$
\inf _{\mu \in M_{1}(F)} J_{\psi}(\mu)
$$

zдe

$$
J_{\psi}(\mu)=\int_{F}\left(3 V_{*}^{\mu}(y)+G_{E}^{\mu}(y)\right) d \mu(y)+6 \int_{F} g_{E}(y, \infty) d \mu(y)
$$

- энергия (удвоенная) мерь $\mu$ с потенциалом $3 V_{*}^{\mu}(z)+G_{E}^{\mu}(z)$ во внешнем поле $\psi(z)=3 g_{E}(z, \infty)$. Стандартными методами теории потенциала (см. [42], [44], [63], [96]) доказывается, что для задачи (95) существует единственная экстремальная мера $\eta_{F} \in M_{1}(F)$ с носителем на $F$, т.е. такая, что

$$
J_{\psi}\left(\eta_{F}\right)=\inf _{\mu \in M_{1}(F)} J_{\psi}(\mu) .
$$

Эта экстремальная мера $\eta_{F}$ является также единственной равновесной мерой для указанного потенииала с внешним полем $\psi$ (см. [42]), т.е. выполняются следующие соотношения равновесия:

$$
3 V_{*}^{\eta_{F}}(y)+G_{E}^{\eta_{F}}(y)+3 g_{E}(y, \infty) \equiv \text { const }=w_{F}, \quad y \in F,
$$

$w_{F}-$ постоянная равновесия.

Докажем, что

$$
\frac{1}{n} \chi\left(Q_{n, 2}\right) \stackrel{*}{\rightarrow} \eta_{F}, \quad n \rightarrow \infty
$$


B соответствии с GRS-методом, предположим, что это не так. Тогда, поскольку все нули полиномов $Q_{n, 2}$, кроме конечного их числа, принадлежат множеству $F$ и $\operatorname{deg} Q_{n, 2} \geqslant n-$ const, где const не зависит от $n$, то по некоторой бесконечной подпоследовательности $\Lambda \subset \mathbb{N}$ имеем:

$$
\frac{1}{n} \chi\left(Q_{n, 2}\right) \stackrel{*}{\rightarrow} \mu^{Q}=\mu \neq \eta_{F}, \quad n \rightarrow \infty, \quad n \in \Lambda,
$$

где $\left|\mu^{Q}\right|=1, \operatorname{supp} \mu^{Q} \subset F$. Поскольку $\mu \neq \eta_{F}, \mu \in M_{1}(F)$ и равновесная мера $\eta_{F}$ единственная (в классе $M_{1}(F)$ ), из предположения (100) вытекает, что для меры $\mu$ соотношения равновесия (98) не могут выполняться, т. е. $3 V_{*}^{\mu}(y)+G_{E}^{\mu}(y)+$ $3 g_{E}(y, \infty) \not \equiv$ const при $y \in F$. Функция $u(z):=3 V_{*}^{\mu}(z)+G_{E}^{\mu}(z)+3 g_{E}(z, \infty)$ полунепрерывна снизу на $F$ и, следовательно, в некоторой точке $y_{0} \in F$ достигает своего минимума на $F: u\left(y_{0}\right)=\min _{y \in F} u(y)=\mathfrak{m}$. При этом, в силу вышесказанного, $u(y) \not \equiv \mathfrak{m}=u\left(y_{0}\right), y \in F$. Далее будем считать, что точка $y_{0}$ лежит внутри $F, y_{0} \in F^{\circ}$, и это единственная точка минимума функции $u(y)$ на $F$, т. е.

$$
\mathfrak{m}=\min _{t \in F} u(t)=u\left(y_{0}\right)<u(y), \quad y \in F \backslash\left\{y_{0}\right\}
$$

Подчеркнем, что эти предположения не ограничивают общности наших дальнейших рассуждений, поскольку общий случай, когда функция $u(y)$ достигает своего минимума на $F$ в нескольких точках или единственная точка минимума совпадает одной из концевых точек $e_{j}$ множества $F$, сводится в рамках GRS-метода к рассмотренному случаю стандартным образом с помощью аналогов леммы 9 из [44] (см. [94; лемма 5]). Выберем теперь полином $\omega_{n}^{*}$ в (89) так, чтобы

$$
\frac{1}{n} \chi\left(\omega_{n}^{*}\right) \stackrel{*}{\rightarrow} \mu^{Q}=\mu, \quad n \rightarrow \infty .
$$

Тогда из соотношений ортогональности (90) для полинома $q_{n, 2}$ стандартным применением GRS-метода получаем (напомним, что $n=2 m$ ), что

$$
\frac{2}{n} \chi\left(q_{n, 2}\right) \rightarrow \nu, \quad n \rightarrow \infty
$$

где $\nu \in M_{1}(E), \nu=\widetilde{\mu}=\mathfrak{b}_{E}(\mu)$ - выметание меры $\mu \in M_{1}(F)$ из области $D$ на границу $\partial D=E$. Тем самым, имеем $V_{*}^{\nu}(z) \equiv V_{*}^{\mu}(z)-G_{E}^{\mu}(z)+$ const, $z \in \overline{\mathbb{C}}$. Поскольку в силу GRS-метода

$$
\left|\int_{E} \frac{q_{n, 2}^{2}(x) d \rho_{n}(x)}{\omega_{n}^{*}(x)(y-x)}\right|^{1 / n} \stackrel{\text { cap }}{\longrightarrow} e^{\text {const }} \neq 0, \infty, \quad y \in F^{0}
$$

то, применяя теперь GRS-метод к выражению (93), определяющему переменный вес $\Psi_{n}$, получаем

$$
\left|\Psi_{n}(y)\right|^{2 / n} \stackrel{\text { cap }}{\longrightarrow} e^{V_{*}^{\nu}(y)-3 g_{E}(z, \infty)}=e^{V_{*}^{\mu}(z)-G_{E}^{\mu}(z)-3 g_{E}(z, \infty)+\text { const }} .
$$


Следовательно, с учетом соотношений $(102),(103)$ и тождества $V_{*}^{\nu}(z) \equiv V_{*}^{\mu}(z)-$ $G_{E}^{\mu}(z)+$ const получаем из $(92)$ предельное соотношение

$$
\begin{aligned}
\left|Q_{n, 2}^{*}(y) \omega_{n}^{*}(y) \Psi_{n}(y)\right|^{2 / n} & \stackrel{\text { cap }}{\longrightarrow} e^{-4 V_{*}^{\mu}(y)+V_{*}^{\nu}(y)-3 g_{E}(y, \infty)} \\
& =e^{-4 V_{*}^{\mu}(y)+V_{*}^{\mu}(y)-G_{E}^{\mu}(y)-3 g_{E}(y, \infty)+\mathrm{const}} \\
& =e^{-3 V_{*}^{\mu}(y)-G_{E}^{\mu}(y)-3 g_{E}(y, \infty)+\mathrm{const}} \\
& =e^{-u(y)+\mathrm{const}} .
\end{aligned}
$$

Тем самым, в силу соотношения $u\left(y_{0}\right)<u(y)$ при $y \in F \backslash\left\{y_{0}\right\}$, модуль подынтегрального выражения в (92), возведенный в степень $2 / n$, имеет в пределе при $n \rightarrow \infty$ единственный строгий максимум на множестве $F$ в точке $y_{0} \in F^{\circ}$. Отсюда с помощью стандартных методов теории потенциала (см., [44; лемма 7], [63]) получаем следующее предельное соотношение:

$$
\begin{aligned}
\left(\int_{F}\left|Q_{n, 2}^{*}(y) \omega_{n}^{*}(y) \Psi_{n}(y)\right||d y|\right)^{2 / n} & \rightarrow e^{-3 V_{*}^{\mu}\left(y_{0}\right)-G_{E}^{\mu}\left(y_{0}\right)-3 g_{E}\left(y_{0}, \infty\right)+\mathrm{const}} \\
& =e^{-u\left(y_{0}\right)+\mathrm{const}} \neq 0 .
\end{aligned}
$$

Вернемся теперь к равенству (94). Наша следующая задача - показать, что существует последовательность полиномов $\omega_{n}^{*}$, для которой сохраняются вышеуказанные свойства (102), (107), но при этом модуль интеграла, стоящего в левой части (94), возведенный в степень $2 / n$, асимптотически ведет себя так же, как левая часть соотношения (107), т. е.

$$
\begin{aligned}
\lim _{\substack{n \rightarrow \infty \\
n \in \Lambda}}\left|\int_{F} Q_{n, 2}^{*}(y) \omega_{n}^{*}(y) \Psi_{n}(y) d y\right|^{2 / n} & =\lim _{\substack{n \rightarrow \infty \\
n \in \Lambda}}\left(\int_{F}\left|Q_{n, 2}^{*}(y) \omega_{n}^{*}(y) \Psi_{n}(y)\right||d y|\right)^{2 / n} \\
& =e^{-u\left(y_{0}\right)+\text { const }} \neq 0 .
\end{aligned}
$$

Очевидно, что получив соотношение (108), мы придем к противоречию с равенством (94).

Предельное распределение нулей полинома $\omega_{n}^{*} \in \mathbb{P}_{n-\text { const }}$, где постоянная const зависит от $q$, но не зависит от $n$, совпадает с предельным распределением нулей полинома $\widetilde{\omega}_{n}$, который отличается от полинома $\omega_{n}^{*}$ отсутствием $k_{n} \in \mathbb{N}$ сомножителей, где $k_{n}=o(n)$ при $n \rightarrow \infty$. Поскольку функция $u$ имеет в точке $y_{0}$ строгий минимум, то, очевидно, $\mu\left(\left\{y_{0}\right\}\right)=0$. Тем самым, существует достаточно малое $\varepsilon>0$ такое, что вне $\varepsilon$-окрестности $U_{\varepsilon}\left(y_{0}\right)$ точки $y_{0}$ число нулей полинома $Q_{n, 2}$ с ростом $n$ неограниченно растет. Зафиксируем это $\varepsilon>0$. Тогда для каждого $n \in \Lambda$ существует монический полином $g_{n}$ фиксированной не зависящей от $n$ степени такой, что выполняются следующие условия: $g_{n}$ делит $Q_{n, 2}^{*}$ и $\omega_{n}^{*}=Q_{n, 2}^{*} / g_{n} \in \mathbb{R}_{n-l}[z] ; g_{n}$ сохраняет знак в $U_{\varepsilon}\left(y_{0}\right)$. Функция $\Psi_{n}(z)$ не зависит от выбора полинома $\widetilde{q}_{n, 2} \in \mathbb{P}_{m-q}$ в (93). Теперь, когда предельное распределение нулей полиномов $q_{n, 2}$ при $n \rightarrow \infty$ установлено и показано, что все они за возможным исключением $o(n)$ лежат на $E$, мы можем удалить соответствующие множители из полинома $\widetilde{q}_{n, 2}$, сделав его знакопостоянным на $F$. Следовательно, соотношение (94) принимает вид

$$
\int_{F} Q_{n, 2}^{* 2}(y) \frac{\Psi_{n}(y)}{g_{n}(y)} d y=0,
$$


причем подынтегральное выражение знакопостоянно на множестве $U_{\varepsilon}\left(y_{0}\right)$; не ограничивая общности, далее будем считать его положительным в $U_{\varepsilon}\left(y_{0}\right)$. Перепишем соотношение (109) в виде

$$
\int_{U_{\varepsilon}\left(y_{0}\right)} Q_{n, 2}^{* 2}(y) \frac{\Psi_{n}(y)}{g_{n}(y)} d y=-\int_{F \backslash \bar{U}_{\varepsilon}\left(y_{0}\right)} Q_{n, 2}^{* 2}(y) \frac{\Psi_{n}(y)}{g_{n}(y)} d y .
$$

Обозначим интеграл, стоящий в левой части равенства (110), через $I_{1}$, а в правой - через $I_{2}$. Из неравенства $u\left(y_{0}\right)<u(y), y \in F \backslash\left\{y_{0}\right\}$, вытекает, что существует предел

$$
\lim _{\substack{n \rightarrow \infty \\ n \in \Lambda}}\left|I_{1}\right|^{2 / n}=\lim _{\substack{n \rightarrow \infty \\ n \in \Lambda}}\left(\int_{U_{\varepsilon}\left(y_{0}\right)}\left|Q_{n, 2}^{* 2}(y) \frac{\Psi_{n}(y)}{g_{n}(y)}\right||d y|\right)^{2 / n}=e^{-u\left(y_{0}\right)}=e^{-\mathfrak{m}} .
$$

Поскольку функция $u$ имеет в точке $y_{0}$ единственный минимум, для интеграла $I_{2}$ получаем

$$
\varlimsup_{\substack{n \rightarrow \infty \\ n \in \Lambda}}\left|I_{2}\right|^{2 / n}<e^{-\mathfrak{m}}
$$

Соотношения (111) и (112) в совокупности противоречат равенству (110). Таким образом, сделанное нами предположение о том, что

$$
\frac{1}{n} \chi\left(Q_{n, 2}\right) \stackrel{*}{\rightarrow} \mu \neq \eta_{F}, \quad n \rightarrow \infty, \quad n \in \Lambda,
$$

привело нас к противоречию. Следовательно, оно неверно. Тем самым, доказано, что существует предел

$$
\lim _{n \rightarrow \infty} \frac{1}{n} \chi\left(Q_{n, 2}\right)=\eta_{F}
$$

Задача о предельном распределении нулей полиномов $Q_{n, 2}$ решена.

Так как полиномы $Q_{n, 0}$ соответствуют полиномам $Q_{n, 2}$, но для функции $1 / f$, т. е. $Q_{n, 0}(z ; f)=Q_{n, 2}(z ; 1 / f)$, то задача о распределении нулей этих полиномов также решена.

3.2. Доказательство утверждений 2) и 3) теоремы 1. Рассмотрим теперь задачу о предельном распределении нулей полиномов $Q_{n, 1}$. Эта задача будет решена одновременно с задачей об интерполяции функции $f_{2}=-\tilde{f}$ рациональной функцией $Q_{n, 1} / Q_{n, 2}$ и о сходимости соответствующей последовательности рациональных функций.

Изучим предельное распределение точек интерполяции. Напомним, что $\widetilde{f}_{j}=\left.\widetilde{f}\right|_{E_{j}^{\circ}}=f^{+}+f^{-}, j=1, \ldots, q, \widetilde{f} \in \mathscr{H}(G)$. Вернемся к соотношению (63). Перепишем его в виде

$$
\int_{E}\left(Q_{n, 1}+Q_{n, 2} \tilde{f}\right)(x) q(x) \Delta f(x) d x=0,
$$

где $q \in \mathbb{P}_{2 n}$ - произвольный полином. Из представления (56) для функции $f$ и условия $f(\infty)=1$ вытекает, что функция $\widetilde{f}$ вещественнозначна на $E$ и для некоторой нетривиальной постоянной const $\neq 0$ выполняется неравенство 
const $\Delta f(x) \geqslant 0$ при $x \in E$. Поскольку $Q_{n, j} \in \mathbb{R}[x]$, то непосредственно из соотношений ортогональности (113) вытекает, что функция $Q_{n, 1}+Q_{n, 2} \tilde{f}$ имеет на $\widehat{E}=\operatorname{conv} E$ по меньшей мере $2 n+1$ простой нуль, причем в каждой лакуне может лежать не более одного нуля. Таким образом, на множестве $E$ эта функция имеет по меньшей мере $2 n+2-q$ простой нуль. Обозначим через $\Omega_{n}(z)=z^{\operatorname{deg} \Omega_{n}}+\cdots$ соответствующий монический полином с простыми нулями в этих точках, тогда $2 n+2-q \leqslant \operatorname{deg} \Omega_{n} \leqslant 2 n+1$. По определению полинома $\Omega_{n}$ функция $\left(Q_{n, 1}+Q_{n, 2} \tilde{f}\right) / \Omega_{n}$ голоморфна на $E^{\circ}$, а следовательно, и в области $G:=\overline{\mathbb{C}} \backslash F$. Значит, для любого полинома $q \in \mathbb{P}_{n-q}$ выполняется равенство

$$
0=\int_{\gamma} \frac{\left(Q_{n, 1}+Q_{n, 2} \tilde{f}\right)(t) q(t) d t}{\Omega_{n}(t)}=\int_{\gamma} \frac{Q_{n, 2}(t) q(t) \tilde{f}(t) d t}{\Omega_{n}(t)},
$$

и справедливо представление

$$
\frac{\left(Q_{n, 1}+Q_{n, 2} \tilde{f}\right)(z)}{\Omega_{n}(z)}=\frac{1}{2 \pi i \widetilde{Q}_{n, 2}(z)} \int_{\gamma} \frac{Q_{n, 2}(t) \widetilde{Q}_{n, 2}(t) \tilde{f}(t) d t}{\Omega_{n}(t)(t-z)}, \quad z \in \operatorname{int} \gamma
$$

где монический полином $\widetilde{Q}_{n, 2}$ отличается от полинома $Q_{n, 2}$ лишь отсутствием конечного числа простых сомножителей, а именно тех, чьи нули лежат вне $F$; в (114) и (115) интегрирование ведется по кривой $\gamma$, состоящей из конечного числа $q$ кривых $\gamma_{j}$, отделяющих нули полинома $\Omega_{n}$ и точку $z$ от бесконечно удаленной точки. С учетом свойств функции $\widetilde{f}$ соотношения (114) и (115) эквивалентным образом переписываются соответственно в виде (ср. [90; формулы (15), (16)]):

$$
\begin{gathered}
\int_{F} \frac{Q_{n, 2}^{*}(y) q(y) \Delta \widetilde{f}(y) d y}{\Omega_{n}(y)}=0 \quad \forall q \in \mathbb{P}_{n-q}, \\
\frac{\left(Q_{n, 1}+Q_{n, 2} \widetilde{f}\right)(z)}{\Omega_{n}(z)}=\frac{1}{2 \pi i \widetilde{Q}_{n, 2}^{*}(z)} \int_{F} \frac{Q_{n, 2}(y) \widetilde{Q}_{n, 2}^{*}(y) \Delta \widetilde{f}(y) d y}{\Omega_{n}(y)(y-z)}, \\
\widetilde{Q}_{n, 2} \in \mathbb{P}_{n-q}, \quad z \notin F
\end{gathered}
$$

(отметим, что $\Delta \widetilde{f}(z)=$ const $f(z), z \in D$, const $\neq 0$ ). Пусть при $n \rightarrow \infty, n \in \Lambda$

$$
\frac{1}{2 n} \chi\left(\Omega_{n}\right) \rightarrow \nu, \quad \operatorname{supp} \nu \subset E, \quad|\nu|=1 .
$$

Тогда в соответствии с GRS-методом соотношения ортогональности (113) и (116) (см. также (118) ниже) дают $n^{-1} \chi\left(Q_{n, 2}\right) \rightarrow \widetilde{\nu} \in M_{1}(F)$, где $\widetilde{\nu}=\mathfrak{b}_{F}(\nu)$ - выметание меры $\nu$ из области $G$ на $F$. Но поскольку нам уже известно, что $n^{-1} \chi\left(Q_{n, 2}\right) \rightarrow \eta_{F}$, получаем, что $\widetilde{\nu}=\eta_{F}$. С помощью представления (117) соотношения ортогональности (113) можно переписать в виде

$$
\int_{E} \Omega_{n}(x) q(x) \Psi_{n}(x) \Delta f(x) d x=0 \quad \forall q \in \mathbb{P}_{2 n-q},
$$

где

$$
\Psi_{n}(z):=\frac{1}{\widetilde{Q}_{n, 2}^{*}(z)} \int_{F} \frac{Q_{n, 2}^{*}(y) \widetilde{Q}_{n, 2}^{*}(y) \Delta \tilde{f}(y) d y}{\Omega_{n}(y)(y-z)}, \quad z \notin F,
$$


- переменный вес, зависящий от самого полинома $\Omega_{n}$. Однако это не мешает нам применить GRS-метод, поскольку мы знаем, что все нули $\Omega_{n}$ лежат на $E$, $2 n+2-q \leqslant \operatorname{deg} \Omega_{n} \leqslant 2 n+1$, и если $(2 n)^{-1} \chi\left(\Omega_{n}\right) \rightarrow \nu, n \rightarrow \infty, n \in \Lambda$, то $\operatorname{supp} \nu \subset E$ и $\widetilde{\nu}=\eta_{F}$. Следовательно, GRS-методом получаем

$$
\left|\Psi_{n}(z)\right|^{1 / n} \stackrel{\text { cap }}{\longrightarrow} e^{V_{*}^{\eta_{F}}(z)+\mathrm{const}}, \quad z \notin F .
$$

Докажем теперь, что если $(2 n)^{-1} \chi\left(\Omega_{n}\right) \rightarrow \nu$, то $\nu=\eta_{E}$, где $\eta_{E} \in M_{1}(E)-$ единственная экстремальная мера для следующей теоретико-потенциальной задачи.

ЗАДАЧА РАВНОВЕСИЯ 2. Пусть $3 V^{\nu}(z)+G_{F}^{\nu}(z)$ - смешанныц гриново-логарифмический потенииал произвольной (положительной борелевской) единичной меры $\nu$ с носителем на $E, \nu \in M_{1}(E) ;$ здесъ $G_{F}^{\nu}(z):=\int_{E} g_{F}(x, z) d \nu(x)-$ гринов (относительно $F$ ) потенциал меры $\nu, g_{F}(x, z)$ - функция Грина для области $G=\overline{\mathbb{C}} \backslash F$. Пусть

$$
\begin{aligned}
J(\nu) & :=\int_{E \times E}\left(3 \log \frac{1}{|x-u|}+g_{F}(x, u)\right) d \nu(x) d \nu(u) \\
& =\int_{E}\left(3 V^{\nu}(x)+G_{F}^{\nu}(x)\right) d \nu(x)
\end{aligned}
$$

- соответствующий функционал энергии. Рассмотрим следующую экстремальную задачу:

$$
\mathfrak{m}=\inf _{\nu \in M_{1}(E)} J(\nu) .
$$

Стандартными методами теории потенциала аналогично работе [42] (см. также [63], [96]) доказывается, что существует единственная (в классе $M_{1}(E)$ ) экстремальная мера $\eta_{E} \in M_{1}(E)$, для которой в (121) достигается минимум:

$$
J\left(\eta_{E}\right)=\min _{\nu \in M_{1}(E)} J(\nu) .
$$

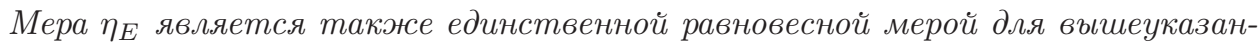
ного потенциала, т.е. для нее выполняются следующие условия равновесия:

$$
3 V^{\eta_{E}}(x)+G_{F}^{\eta_{E}}(x) \equiv \mathrm{const}=w_{E}, \quad x \in E,
$$

$w_{E}$ - постоянная равновесия. В работе [25] (см. также [23], [72]) показано, что $\widetilde{\eta}_{E}=\mathfrak{b}_{F}\left(\eta_{E}\right)=\eta_{F}\left(\right.$ отсюда, в частности, вытекает, что $\left.\operatorname{supp} \eta_{F}=F\right)$. Следовательно, имеем $V^{\eta_{F}}(z) \equiv V_{*}^{\eta_{E}}(z)-G_{F}^{\eta_{E}}(z)+$ const, $z \in \overline{\mathbb{C}}$.

Из вещественной симметрии рассматриваемой в настоящей работе задачи и того факта, что $\operatorname{supp} \eta_{E} \subset E$ и $\operatorname{supp} \eta_{F} \subset F$, вытекает, что

$$
\frac{\partial\left(V^{\eta_{E}}-V_{*}^{\eta_{F}}\right)}{\partial n^{+}}(x)=\frac{\partial\left(V^{\eta_{E}}-V_{*}^{\eta_{F}}\right)}{\partial n^{-}}(x), \quad x \in E^{\circ},
$$

т. е. компакт $E$ является $S$-кривой во внешнем поле, порожденном потенциалом заряда $-\eta_{F}$. Полиномы $\Omega_{n}$ квазиортогональны (см. (118)) на $E$ относительно 
переменного веса $\Psi_{n}$, для которого справедливо асимптотическое соотношение (120). С учетом того, что $\operatorname{deg} \Omega_{n} / n \rightarrow 2$ при $n \rightarrow \infty$, непосредственно из теоремы Гончара-Рахманова [44; теорема 3$]$, частного случая общего GRS-метода, вытекает, что предельное распределение нулей полиномов $\Omega_{n}$ существует и совпадает с мерой $\mu^{\mathrm{eq}} \in M_{1}(E)$, равновесной на $E$ во внешнем поле $\psi(z)=-(1 / 4) V_{*}^{\eta_{F}}(z)$, т. е.

$$
\frac{1}{2 n} \chi\left(\Omega_{n}\right) \rightarrow \mu^{\mathrm{eq}} \in M_{1}(E), \quad n \rightarrow \infty,
$$

где $\mu^{\mathrm{eq}} \in M_{1}(E)$ - единственная мера такая, что

$$
V_{*}^{\mu^{\mathrm{eq}}}(x)-\frac{1}{4} V_{*}^{\eta_{F}}(x) \equiv \text { const }, \quad x \in E .
$$

С учетом того, что $\eta_{F}=\widetilde{\eta}_{E}$ и, следовательно, $V^{\eta_{F}}(z)=V_{*}^{\eta_{E}}(z)-G_{F}^{\eta_{E}}(z)+$ const, соотношение (123) очевидно эквивалентно следующему соотношению равновесия:

$$
4 V_{*}^{\mu^{\mathrm{eq}}}(x)-V^{\eta_{E}}(x)+G_{F}^{\eta_{E}}(x) \equiv \text { const }, \quad x \in E .
$$

Поскольку решение задачи равновесия (123) единственно, решение задачи (124) тоже единственно. Непосредственно из (122) и (123) вытекает, что $\mu^{\mathrm{eq}}=\eta_{E}$. Тем самым, доказано, что

$$
\frac{1}{2 n} \chi\left(\Omega_{n}\right) \stackrel{*}{\rightarrow} \eta_{E}, \quad n \rightarrow \infty .
$$

Таким образом, предельное распределение точек интерполяции функции $\tilde{f}$ рациональной функцией $-Q_{n, 1} / Q_{n, 2}$ на множестве $E$ существует и совпадает с мерой $\eta_{E}$, равновесной для задачи (122).

Теперь уже непосредственно из (117), применяя GRS-метод, получаем, что

$$
\left|\tilde{f}(z)+\frac{Q_{n, 1}}{Q_{n, 2}}(z)\right|^{1 / n} \stackrel{\text { cap }}{\longrightarrow} e^{2\left(V^{\eta_{F}}(z)-V_{*}^{\eta_{E}}(z)-\text { const }\right)}=e^{-2 G_{F}^{\eta_{E}}(z)}<1, \quad z \notin \mathbb{R}
$$

(нетрудно увидеть, что постоянная const в (125) та же самая, что и в тождестве $V_{*}^{\eta_{F}}(z)=V^{\eta_{E}}(z)-G_{F}^{\eta_{E}}(z)+$ const). Неравенство (54) вытекает из (117) и принципа понижения для логарифмического потенциала (см. [63]). Теорема 1 доказана.

ЗАмЕчАниЕ 7 . Если $\alpha \in \mathbb{C},|\alpha| \in(0,1 / 2)$, то все предыдущие результаты об асимптотическом поведении выражения $\left|Q_{n, 2}^{*}(y) \omega_{n}^{*}(y) \Psi_{n}(y)\right|^{1 / n}$ остаются справедливыми. Свобода в выборе второго сомножителя $\omega_{n, 2}$ малой степени $k_{n}=o(n)$ в полиноме $\omega_{n}^{*}$ оказывается вполне достаточной для того, чтобы, оставаясь в рамках GRS-метода, подправить аргумент в произведении $Q_{n, 2}^{*}(y) \omega_{n}^{*}(y) \Psi_{n}(y)$ в произвольной достаточно малой (но фиксированной) окрестности точки асимптотического максимума $y_{0} \in F^{\circ}$ таким образом, чтобы при $n \rightarrow \infty$ асимптотики двух интегралов

$$
\left(\int_{F}\left|Q_{n, 2}^{*}(y) \omega_{n}^{*}(y) \Psi_{n}(y)\right||d y|\right)^{2 / n} \quad \text { и }\left|\int_{F} Q_{n, 2}^{*}(y) \omega_{n}^{*}(y) \Psi_{n}(y) d y\right|^{2 / n}
$$


совпадали друг с другом (подробнее см. [21], [44; лемма 9], [94; лемма 5]). После того как это совпадение установлено, мы придем к существованию следующего предела:

$$
\lim _{\substack{n \rightarrow \infty \\ n \in \Lambda}}\left|\int_{F} Q_{n, 2}^{*}(y) \omega_{n}^{*}(y) \Psi_{n}(y) d y\right|^{2 / n}=e^{-3 V_{*}^{\mu}\left(y_{0}\right)-G_{E}^{\mu}\left(y_{0}\right)-3 g_{E}\left(y_{0}, \infty\right)+\text { const }} \neq 0 .
$$

Как и раньше, равенство (127) будет противоречить соотношению ортогональности (94). Таким образом, окажется, что сделанное предположение о том, что $\mu^{Q}=\mu \neq \eta_{F}$, приводит нас к противоречию. Следовательно,

$$
\frac{1}{n} \chi\left(Q_{n, 2}^{*}\right) \rightarrow \eta_{F}, \quad n \rightarrow \infty \text {. }
$$

\section{Список литературы}

[1] Н. И. Ахиезер, "Об ортогональных многочленах на нескольких интервалах", Докл. АН ССCP, 134:1 (1960), 9-12; англ. пер.: N.I. Akhiezer, "Orthogonal polynomials on several intervals", Soviet Math. Dokl., 1 (1960), 989-992.

[2] C. M. Andersen, J.F. Geer, "Power series expansions for the frequency and period of the limit cycle of the van der Pol equation", SIAM J. Appl. Math., 42:3 (1982), 678-693.

[3] И. Андрианов, Я. Аврейцевич, Методъ асимптотического анализа и синтеза в нелинейной динамике и механике деформируемого твердого тела, ИКИ, М.-Ижевск, 2013, 276 с.

[4] А.И. Аптекарев, "Точные константы рациональных аппроксимаций аналитических функций", Матем. сб., 193:1 (2002), 3-72; англ. пер.: А. I. Aptekarev, "Sharp constants for rational approximations of analytic functions", Sb. Math., 193:1 (2002), 1-72.

[5] А. И. Аптекарев, "Асимптотика аппроксимаций Эрмита-Паде для пары функций с точками ветвления", Докл. РАН, 422:4 (2008), 443-445; англ. пер.: A. I. Aptekarev, "Asymptotics of Hermite-Padé approximants for two functions with branch points", Dokl. Math., 78:2 (2008), 717-719.

[6] А. И. Аптекарев, В. И. Буслаев, А. Мартинес-Финкельштейн, С. П. Суетин, "Аппроксимации Паде, непрерывные дроби и ортогональные многочлены", УМН, 66:6(402) (2011), 37-122; англ. пер.: A. I. Aptekarev, V. I. Buslaev, A. Martínez-Finkelshtein, S.P. Suetin, "Padé approximants, continued fractions, and orthogonal polynomials", Russian Math. Surveys, 66:6 (2011), 1049-1131.

[7] А. И. Аптекарев, А.Э. Койэлаарс, "Аппроксимации Эрмита-Паде и ансамбли совместно ортогональных многочленов”, УМН, 66:6(402) (2011), 123-190; англ. пер.: A.I. Aptekarev, A. Kuijlaars, "Hermite-Padé approximations and multiple orthogonal polynomial ensembles", Russian Math. Surveys, 66:6 (2011), 1133-1199.

[8] A. I. Aptekarev, A. B. J. Kuijlaars, W. Van Assche, "Asymptotics of Hermite-Padé rational approximants for two analytic functions with separated pairs of branch points (case of genus 0)", Int. Math. Res. Pap. IMRP, 2008:4 (2008), rpm007, 128 c.

[9] А.И. Аптекарев, В.Г. Лысов, "Системы марковских функций, генерируемые графами, и асимптотика их аппроксимаций Эрмита-Паде", Матем. сб., 201:2 (2010), 29-78; англ. пер.: A. I. Aptekarev, V. G. Lysov, "Systems of Markov functions generated by graphs and the asymptotics of their Hermite-Padé approximants", Sb. Math., 201:2 (2010), 183-234. 
[10] А. И. Аптекарев, В. Г. Лысов, Д.Н. Туляков, "Случайные матрицы с внешним источником и асимптотика совместно ортогональных многочленов", Матем. сб., 202:2 (2011), 3-56; англ. пер.: А. I. Aptekarev, V. G. Lysov, D. N. Tulyakov, "Random matrices with external source and the asymptotic behaviour of multiple orthogonal polynomials", Sb. Math., 202:2 (2011), 155-206.

[11] A.I. Aptekarev, D. N. Tulyakov, "Geometry of Hermite-Padé approximants for system of functions $\left\{f, f^{2}\right\}$ with three branch points", Препринты ИПМ им. М.В. Келдиша, 2012, 077, 25 с.

[12] А.И. Аптекарев, Д.Н. Туляков, “Абелев интеграл Наттолла на римановой поверхности кубического корня многочлена 3-й степени", Препринты ИПМ им. М.В. Келдиша, 2014, 015, 25 с.

[13] A. I. Aptekarev, W. Van Assche, M. L. Yattselev, Hermite-Padé approximants for a pair of Cauchy transforms with overlapping symmetric supports, 2015, 52 pp., arXiv: 1505.03993.

[14] A.I. Aptekarev, M. L. Yattselev, Padé approximants for functions with branch points - strong asymptotics of Nuttall-Stahl polynomials, 2011 (v2 - 2012), 47 pp., arXiv: 1109.0332.

[15] Н. У. Аракелян, "Об эффективном аналитическом продолжении степенных рядов", Матем. сб., 124(166):1(5) (1984), 24-44; англ. пер.: N. U. Arakelian, "On efficient analytic continuation of power series", Math. USSR-Sb., 52:1 (1985), 21-39.

[16] S. S. Baghsorkhi, S. P. Suetin, Embedding AC power flow with voltage control in the complex plane: the case of analytic continuation via Padé approximants, 2015, 9 pp., arXiv: 1504.03249.

[17] G. A. Baker, Jr., P. Graves-Morris, Padé approximants, 2nd ed., Encyclopedia Math. Appl., 59, Addison-Wesley Publishing Co., Reading, MA, 1996, xiv+746 c.; pyc. пер. 1-го изд.: Дж. Бейкер мл., П. Грейвс-Моррис, Аппроксимации Паде, Мир, М., 1986, 502 с.

[18] Л. Бибербах, Аналитическое продолжение, Наука, М., 1967, 240 с.; пер. с нем.: L. Bieberbach, Analytische Fortsetzung, Ergeb. Math. Grenzgeb. (N. F.), 3, SpringerVerlag, Berlin-Göttingen-Heidelberg, 1955, ii+168 pp.

[19] É. Borel, Leçons sur les fonctions de variables réelles et les développements en séries de polynômes, Gauthier-Villars, Paris, 1905, viii+158 pp.

[20] В.И. Буслаев, "О сходимости непрерывных Т-дробей", Аналитические и геометрические вопросы комплексного анализа, Сборник статей. K 70-летию со дня рождения академика Анатолия Георгиевича Витушкина, Тр. МИАН, 235, Наука, М., 2001, 36-51; англ. пер.: V. I. Buslaev, "On the convergence of continued T-fractions", Proc. Steklov Inst. Math., 235 (2001), 29-43.

[21] В. И. Буслаев, "О сходимости многоточечных аппроксимаций Паде кусочно аналитических функций”, Матем. сб., 204:2 (2013), 39-72; англ. пер.: V. I. Buslaev, "Convergence of multipoint Padé approximants of piecewise analytic functions", $S b$. Math., 204:2 (2013), 190-222.

[22] В. И. Буслаев, “О сходимости $m$-точечных аппроксимаций Паде набора многозначных аналитических функций”, Матем. сб., 206:2 (2015), 5-30; англ. пер.: V. I. Buslaev, "Convergence of $m$-point Padé approximants of a tuple of multivalued analytic functions", Sb. Math., 206:2 (2015), 175-200.

[23] В. И. Буслаев, А. Мартинес-Финкельштейн, С. П. Суетин, "Метод внутренних вариаций и существование $S$-компактов", Аналитические и геометрические вопросы комплексного анализа, Сборник статей, Тр. МИАН, 279, МАИК, М., 2012, 31-58; англ. пер.: V. I. Buslaev, A. Martínez-Finkelshtein, S. P. Suetin, "Method of interior variations and existence of S-compact sets", Proc. Steklov Inst. Math., 279 (2012), 25-51. 
[24] В. И. Буслаев, С. П. Суетин, "Об одной теоретико-потенциальной задаче равновесия", УМH, 69:5(419) (2014), 157-158; англ. пер.: V. I. Buslaev, S. P. Suetin, "An extremal problem in potential theory", Russian Math. Surveys, 69:5 (2014), 915-917.

[25] В. И. Буслаев, С. П. Суетин, "О задачах равновесия, связанных с распределением нулей полиномов Эрмита-Паде", Современные проблемы математики, механики и математической физики, Сборник статей, Тр. МИАН, 290, МАИК, M., 2015, 272-279.

[26] П.Л. Чебышев, “О непрерывных дробях", Ученые зап. Имп. акад. наук, III (1855), 636-664; Полное собрание сочинений, т. II, Изд-во АН СССР, М.-Л., 1948, 103-126; фр. пер.: P. Tchébycheff, "Sur les fractions continues", J. Math. Pures Appl. (2), 3 (1858), 289-323.

[27] D. V. Chudnovsky, G. V. Chudnovsky, "Padé approximations to solutions of linear differential equations and applications to Diophantine analysis", Number theory (New York, 1982), Lecture Notes in Math., 1052, Springer, Berlin, 1984, 85-167.

[28] D. V. Chudnovsky, G. V. Chudnovsky, "The Wronskian formalism for linear differential equations and Padé approximations", Adv. in Math., 53:1 (1984), 28-54.

[29] G. V. Chudnovsky, "Padé approximations to the generalized hypergeometric functions. I", J. Math. Pures Appl. (9), 58:4 (1979), 445-476.

[30] G. V. Chudnovsky, "Padé approximation and the Riemann monodromy problem", Bifurcation phenomena in mathematical physics and related topics (Cargèse, 1979), NATO Adv. Study Inst. Ser., Ser. C: Math. Phys. Sci., 54, Reidel, Dordrecht-Boston, MA, 1980, 449-510.

[31] M. B. Dadfar, J. Geer, C. M. Andersen, "Perturbation analysis of the limit cycle of the free van der Pol equation", SIAM J. Appl. Math., 44:5 (1984), 881-895.

[32] A. Deaño, D. Huybrechs, A. B. J. Kuijlaars, "Asymptotic zero distribution of complex orthogonal polynomials associated with Gaussian quadrature", J. Approx. Theory, 162:12 (2010), 2202-2224; 2010, 33 pp., arXiv: 1001.2219.

[33] С. Дельво, А. Лопес, Г. Лопес Лагомасино, "Об одном семействе систем Никишина с периодическими рекуррентными коэффициентами”, Матем. сб., 204:1 (2013), 47-78; англ. пер.: S. Delvaux, A. López, G. López Lagomasino, "A family of Nikishin systems with periodic recurrence coefficients", Sb. Math., 204:1 (2013), $43-74$.

[34] V. Druskin, S. Güttel, L. Knizhnerman, "Near-optimal perfectly matched layers for indefinite Helmholtz problems", SIAM Rev., 2016 (to appear); 2014 (v1 - 2013), 27 pp., http://eprints.ma.man.ac.uk/2229; 2015, arXiv: 1507.06265.

[35] S. Dumas, Sur le développement des fonctions elliptiques en fractions continues, Thèse, Zürich, 1908, 59 pp.

[36] U. Fidalgo Prieto, G. López Lagomasino, "Nikishin systems are perfect", Constr. Approx., 34:3 (2011), 297-356.

[37] G. Filipuk, W. Van Assche, Lun Zhang, "Ladder operators and differential equations for multiple orthogonal polynomials", J. Phys. A, 46:20 (2013), 205204, 24 pp.

[38] M. Froissart, "Approximation de Padé: application à la physique des particules élémentaires", Recherche Cooperative sur Programme № 25, v. 9, eds. J. Carmona, M. Froissart, D. W. Robinson, D. Ruelle, Centre National de la Recherche Scientifique (CNRS), Strasbourg, 1969, 1-13.

[39] А. А. Гончар, "5.6. Рациональная аппроксимация аналитических функций”, Исследования по линейным операторам и теории функиий, 99 нерешенных задач 
линейного и комплексного анализа, Зап. науч. сем. ЛОМИ, 81, Изд-во "Наука", Ленингр. отд., Л., 1978, 182-185; англ. пер.: А. А. Gonchar, "5.6. Rational approximation of analytic functions", J. Soviet Math., 26:5 (1984), 2218-2220.

[40] А. А. Гончар, "Рациональные аппроксимации аналитических функций", Совр. пробл. матем., 1, МИАН, М., 2003, 83-106; англ. пер.: А. A. Gonchar, "Rational approximation of analytic functions", Proc. Steklov Inst. Math., 272, suppl. 2 (2011), S44-S57.

[41] А. А. Гончар, Е. А. Рахманов, "О сходимости совместных аппроксимаций Паде для систем функций марковского типа", Теория чисел, математический анализ u ux приложения, Сборник статей. Посвящается академику Ивану Матвеевичу Виноградову к его девяностолетию, Тр. МИАН СССР, 157, 1981, 31-48; англ. пер.: А. A. Gonchar, E. A. Rakhmanov, "On the convergence of simultaneous Padé approximants for systems of functions of Markov type", Proc. Steklov Inst. Math., 157 (1983), 31-50.

[42] А. А. Гончар, Е.А. Рахманов, "Равновесная мера и распределение нулей экстремальных многочленов", Матем. сб., 125(167):1(9) (1984), 117-127; англ. пер.: A. A. Gonchar, E. A. Rakhmanov, "Equilibrium measure and the distribution of zeros of extremal polynomials", Math. USSR-Sb., 53:1 (1986), 119-130.

[43] А. А. Гончар, Е. А. Рахманов, "О задаче равновесия для векторных потенциалов", УМH, 40:4(244) (1985), 155-156; англ. пер.: А. A. Gonchar, E. A. Rakhmanov, "On the equilibrium problem for vector potentials", Russian Math. Surveys, 40:4 (1985), 183-184.

[44] А. А. Гончар, Е.А. Рахманов, "Равновесные распределения и скорость рациональной аппроксимации аналитических функций”, Матем. сб., 134(176):3(11) (1987), 306-352; англ. пер.: А. А. Gonchar, E. А. Rakhmanov, "Equilibrium distributions and degree of rational approximation of analytic functions", Math. USSR-Sb., 62:2 (1989), 305-348.

[45] А. А. Гончар, Е. А. Рахманов, В. Н. Сорокин, "Об аппроксимациях Эрмита-Паде для систем функций марковского типа", Матем. сб., 188:5 (1997), 33-58; англ. пер.: A. A. Gonchar, E. A. Rakhmanov, V. N. Sorokin, "Hermite-Padé approximants for systems of Markov-type functions", Sb. Math., 188:5 (1997), 671-696.

[46] С. О. Горчинский, Д. В. Осипов, “Многомерный символ Конту-Каррера: локальная теория", Матем. сб., 206:9 (2015), 21-98.

[47] S. Gorchinskiy, A. Ovchinnikov, "Isomonodromic differential equations and differential categories", J. Math. Pures Appl. (9), 102:1 (2014), 48-78.

[48] G. H. Halphen, Traité des fonctions elliptiques et de leurs applications. I, GauthierVillars, Paris, 1886, viii+492 pp.

[49] M. Huttner, "Constructible sets of linear differential equations and effective rational approximations of polylogarithmic functions", Israel J. Math., 153 (2006), 1-43.

[50] N. R. Ikonomov, R. K. Kovacheva, S. P. Suetin, Some numerical results on the behavior of zeros of the Hermite-Padé polynomials, 2015, 95 pp., arXiv: 1501.07090.

[51] N. R. Ikonomov, R. K. Kovacheva, S. P. Suetin, On the limit zero distribution of type I Hermite-Padé polynomials, 2015, 67 pp., arXiv: 1506.08031.

[52] П. Джоунс, У. Трон, Непрерывные дроби, Мир, М., 1985, 416 с.; пер. с англ.: W.B. Jones, W. J. Thron, Continued fractions. Analytic theory and applications, Encyclopedia Math. Appl., 11, Addison-Wesley Publishing Co., Reading, MA, 1980, xxix $+428 \mathrm{pp}$.

[53] E. A. Karabut, A. A. Kuzhuget, "Conformal mapping, Padé approximants, and an example of flow with a significant deformation of the free boundary", European J. Appl. Math., 25:6 (2014), 729-747. 
[54] А. Г. Хованский, Топологическая теория Галуа. Разрешимость и неразрешимость уравнений в конечном виде, МЦНМО, М., 2008, 296 с.; англ. пер. A. Khovanskii, Topological Galois theory. Solvability and unsolvability of equations in finite terms, Appendices C and D by Khovanskii and Yu. Burda, Springer Monogr. Math., Springer, Heidelberg, 2014, xviii+307 pp.

[55] E. R. Kolchin, "Rational approximation to solutions of algebraic differential equations", Proc. Amer. Math. Soc., 10:2 (1959), 238-244.

[56] А.В. Комлов, С.П. Суетин, "Асимптотическая формула для двухточечного аналога полиномов Якоби", УМН, 68:4(412) (2013), 183-184; англ. пер.: A. V. Komlov, S.P. Suetin, "An asymptotic formula for a two-point analogue of Jacobi polynomials", Russian Math. Surveys, 68:4 (2013), 779-781.

[57] А. В. Комлов, С. П. Суетин, "Сильная асимптотика двухточечных аппроксимаций Паде многозначных функций степенного вида", Докл. РАН, 455:2 (2014), 138-141; англ. пер.: A. V. Komlov, S. P. Suetin, "Strong asymptotics of two-point Padé approximants for power-like multivalued functions", Dokl. Math., 89:2 (2014), $165-168$.

[58] А. В. Комлов, С. П. Суетин, "Асимптотическая формула для полиномов, ортонормированных относительно переменного веса. II", Матем. сб., 205:9 (2014), 121-144; англ. пер.: A. V. Komlov, S.P. Suetin, "An asymptotic formula for polynomials orthonormal with respect to a varying weight. II", Sb. Math., 205:9 (2014), 1334-1356.

[59] Р. К. Ковачева, С. П. Суетин, "Распределение нулей полиномов Эрмита-Паде для системы из трех функций и конденсатор Наттолла", Функиионалъные пространства и смежные вопросы анализа, Сборник статей. K 80-летию со дня рождения члена-корреспондента РАН Олега Владимировича Бесова, Тр. МИАН, 284, МАИК, М., 2014, 176-199; англ. пер.: R. K. Kovacheva, S. P. Suetin, "Distribution of zeros of the Hermite-Padé polynomials for a system of three functions, and the Nuttall condenser", Proc. Steklov Inst. Math., 284 (2014), $168-191$.

[60] A. B. J. Kuijlaars, G. L. F. Silva, "S-curves in polynomial external fields", J. Approx. Theory, 191 (2015), 1-37.

[61] Г. В. Кузьмина, Модули семейств кривых и квадратичные дифференциалы, Тр. МИАН СССР, 139, 1980; англ. пер.: G. V. Kuz'mina, "Moduli of families of curves and quadratic differentials", Proc. Steklov Inst. Math., 139 (1982), 1-231.

[62] E. N. Laguerre, "Sur la réduction en fractions continues d'une fraction qui satisfait à une équation différentielle linéaire du premier ordre dont les coefficients sont rationnels", J. Math. Pures Appl. (4), 1 (1885), 135-165; Euvres, v. II: Géométrie, Gauthier-Villars, Paris, 1905, 438-448.

[63] Н. С. Ландкоф, Основы современной теории потенииала, Наука, М., 1966, 515 с.; англ. пер.: N.S. Landkof, Foundations of modern potential theory, Grundlehren Math. Wiss., 180, Springer-Verlag, New York-Heidelberg, 1972, x+424 pp.

[64] A. Lasjaunias, "A survey of Diophantine approximation in fields of power series", Monatsh. Math., 130:3 (2000), 211-229.

[65] Г. Лопес Лагомасино, С. Медина Перальта, У. Фидальго Прието, “Аппроксимации Эрмита-Паде для некоторых систем мероморфных функций”, Матем. сб., 206:2 (2015), 57-76; англ. пер.: G. López Lagomasino, S. Medina Peralta, U. Fidalgo Prieto, "Hermite-Padé approximation for certain systems of meromorphic functions", Sb. Math., 206:2 (2015), 225-241.

[66] D. S. Lubinsky, A. Sidi, H. Stahl, "Asymptotic zero distribution of biorthogonal polynomials", J. Approx. Theory, 190 (2015), 26-49. 
[67] A. P. Magnus, J. Nuttall, On the constructive rational approximation of certain entire functions, Draft of preliminary report, not in form intended for publication, 1988, 33 pp., http://publish.uwo.ca/ jnuttall/cafe.pdf.

[68] K. Mahler, "Perfect systems", Compositio Math., 19 (1968), 95-166.

[69] А.И. Маркушевич, Теория аналитических функиий, т. II: Дальнейшее построение теории, 2-е изд., Наука, М., 1968, 624 с.; англ. пер. 1-го изд.: A. I. Markushevich, Theory of functions of a complex variable, v. II, Prentice-Hall, Inc., Englewood Cliffs, NJ, 1965, xii+333 pp.

[70] A. Martínez-Finkelshtein, E. A. Rakhmanov, "On asymptotic behavior of HeineStieltjes and Van Vleck polynomials", Recent trends in orthogonal polynomials and approximation theory, Contemp. Math., 507, Amer. Math. Soc., Providence, RI, 2010, 209-232.

[71] A. Martínez-Finkelshtein, E. A. Rakhmanov, "Critical measures, quadratic differentials, and weak limits of zeros of Stieltjes polynomials", Comm. Math. Phys., 302:1 (2011), 53-111.

[72] А. Мартинес-Финкельштейн, Е.А. Рахманов, С.П. Суетин, "Вариация равновесной меры и $S$-свойство стационарного компакта", УМH, 66:1(397) (2011), 183-184; англ. пер.: A. Martínez-Finkelshtein, E. A. Rakhmanov, S.P. Suetin, "Variation of the equilibrium measure and the $S$-property of a stationary compact set", Russian Math. Surveys, 66:1 (2011), 176-178.

[73] А. Мартинес-Финкельштейн, Е. А. Рахманов, С. П. Суетин, "Вариация равновесной энергии и $S$-свойство стационарного компакта", Матем. сб., 202:12 (2011), 113-136; англ. пер.: A. Martínez-Finkelshtein, E. A. Rakhmanov, S.P. Suetin, "Variation of the equilibrium energy and the $S$-property of stationary compact sets", Sb. Math., 202:12 (2011), 1831-1852.

[74] A. Martínez-Finkelshtein, E. A. Rakhmanov, S. P. Suetin, "Heine, Hilbert, Padé, Riemann, and Stieltjes: John Nuttall's work 25 years later", Recent advances in orthogonal polynomials, special functions, and their applications, Contemp. Math., 578, Amer. Math. Soc., Providence, RI, 2012, 165-193.

[75] А. Мартинес-Финкельштейн, Е.А. Рахманов, С. П. Суетин, "Дифференциальное уравнение для полиномов Эрмита-Паде", УМН, 68:1(409) (2013), 197-198; англ. пер.: A. Martínez-Finkelshtein, E. A. Rakhmanov, S. P. Suetin, "A differential equation for Hermite-Padé polynomials", Russian Math. Surveys, 68:1 (2013), $183-185$.

[76] A. Martínez-Finkelshtein, E. A. Rakhmanov, S. P. Suetin, "Asymptotics of type I Hermite-Padé polynomials for semiclassical functions", Contemp. Math. (to appear).

[77] J. J. Morales-Ruiz, "Picard-Vessiot theory and integrability", J. Geom. Phys., 87 (2015), 314-343.

[78] Е.М. Никишин, "Об асимптотике линейных форм для совместных аппроксимаций Паде", Изв. вузов. Матем., 1986, № 2, 33-41; англ. пер.: E. M. Nikishin, "The asymptotic behavior of linear forms for joint Padé approximations", Soviet Math. (Iz. VUZ), 30:2 (1986), 43-52.

[79] Е. М. Никишин, В.Н. Сорокин, Рациональные аппроксимачии и ортогональность, Наука, М., 1988, 256 с.; англ. пер.: E. M. Nikishin, V. N. Sorokin, Rational approximations and orthogonality, Transl. Math. Monogr., 92, Amer. Math. Soc., Providence, RI, 1991, viii+221 pp.

[80] J. Nuttall, "Sets of minimum capacity, Padé approximants and the bubble problem", Bifurcation phenomena in mathematical physics and related topics, NATO Adv. Study Inst. Ser. C: Math. Phys. Sci., 54, eds. C. Bardos, D. Bessis, D. Reidel Publishing Co., Dordrecht-Boston, MA, 1980, 185-201. 
[81] J. Nuttall, "Asymptotics of diagonal Hermite-Padé polynomials", J. Approx. Theory, 42:4 (1984), 299-386.

[82] J. Nuttall, "Asymptotics of generalized Jacobi polynomials", Constr. Approx., 2:1 (1986), 59-77.

[83] Ch.F. Osgood, "Effective bounds on the 'Diophantine approximation' of algebraic functions over fields of arbitrary characteristic and applications to differential equations", Nederl. Akad. Wetensch. Proc. Ser. A, 78, = Indag. Math., 37 (1975), 105-119.

[84] Ch. F. Osgood, "The approximation of solutions to linear homogeneous differential equations by rational functions", Monatsh. Math., 90:2 (1980), 143-151.

[85] Ch.F. Osgood, "Sometimes effective Thue-Siegel-Roth-Schmidt-Nevanlinna bounds, or better", J. Number Theory, 21:3 (1985), 347-389.

[86] Ch.F. Osgood, "The Diophantine approximation of general solutions of algebraic differential equations", Complex Variables Theory Appl., 43:3-4 (2001), 381-390.

[87] Ch. F. Osgood, "Nevanlinna theory, Diophantine approximation, and numerical analysis", J. Fourier Anal. Appl., 7:3 (2001), 309-317.

[88] Е. А. Перевозникова, Е. А. Рахманов, Вариация равновесной энергии и S-свойство компактов минимальной емкости, Препринт, М., 1994.

[89] O. Perron, Die Lehre von den Kettenbrüchen, v. II: Analytisch-funktionentheoretische Kettenbrüche, 3. verbesserte und erweiterte Aufl., B. G. Teubner Verlagsgesellschaft, Stuttgart, 1957, vi+316 pp.

[90] Е. А. Рахманов, "K асимптотике многочленов Эрмита-Паде для двух марковских функций”, Матем. сб., 202:1 (2011), 133-140; англ. пер.: Е. A. Rakhmanov, "The asymptotics of Hermite-Padé polynomials for two Markov-type functions", $S b$. Math., 202:1 (2011), 127-134.

[91] E. A. Rakhmanov, "Orthogonal polynomials and $S$-curves", Recent advances in orthogonal polynomials, special functions and their applications, Contemp. Math., 578, Amer. Math. Soc., Providence, RI, 2012, 195-239.

[92] E. A. Rakhmanov, "Gonchar-Stahl's $\rho^{2}$-theorem and associated directions in the theory of rational approximation of analytic functions", Матем. сб. (в печати); 2015, 30 pp., arXiv: 1503.06620.

[93] Е.А. Рахманов, С.П. Суетин, “Асимптотика полиномов Эрмита-Паде I рода для пары функций, образующих систему Никишина”, УМН, 67:5(407) (2012), 177-178; англ. пер.: E. A. Rakhmanov, S. P. Suetin, "Asymptotic behaviour of the Hermite-Padé polynomials of the 1st kind for a pair of functions forming a Nikishin system", Russian Math. Surveys, 67:5 (2012), 954-956.

[94] Е. А. Рахманов, С. П. Суетин, "Распределение нулей полиномов Эрмита-Паде для пары функций, образующей систему Никишина", Матем. сб., 204:9 (2013), 115-160; англ. пер.: E. A. Rakhmanov, S. P. Suetin, "The distribution of the zeros of the Hermite-Padé polynomials for a pair of functions forming a Nikishin system", Sb. Math., 204:9 (2013), 1347-1390.

[95] А. К. Рамазанов, “Формулы для рациональных интерполяций и остатков", $M a$ тем. заметки, 96:5 (2014), 762-772; англ. пер.: А. K. Ramazanov, "Formulas for rational interpolation and remainders", Math. Notes, 96:5 (2014), 767-776.

[96] E. B. Saff, V. Totik, Logarithmic potentials with external fields, Appendix B by Th. Bloom, Grundlehren Math. Wiss., 316, Springer-Verlag, Berlin, 1997, xvi+505 pp.

[97] W. M. Schmidt, "Rational approximation to solutions of linear differential equations with algebraic coefficients", Proc. Amer. Math. Soc., 53:2 (1975), 285-289. 
[98] W.M. Schmidt, "On Osgood's effective Thue theorem for algebraic functions", Comm. Pure Appl. Math., 29:6 (1976), 759-773.

[99] W. M. Schmidt, "On continued fractions and Diophantine approximation in power series fields", Acta Arith., 95:2 (2000), 139-166.

[100] W. Y. Sit, "The Ritt-Kolchin theory for differential polynomials", Differential algebra and related topics (Newark, NJ, 2000), World Sci. Publ., River Edge, NJ, 2002, $1-70$.

[101] В.Н. Сорокин, “Аппроксимации Эрмита-Паде последовательных степеней логарифма и их арифметические приложения”, Изв. вузов. Матем., 1991, №11, 66-74; англ. пер.: V. N. Sorokin, "Hermite-Padé approximations of sequential powers of a logarithm and their arithmetic applications", Soviet Math. (Iz. VUZ), 35:11 (1991), 67-74.

[102] В.Н. Сорокин, “Аппроксимации Эрмита-Паде полилогарифмов”, Изв. вузов. Матем., 1994, № 5, 49-59; англ. пер.: V. N. Sorokin, "Hermite-Padé approximants of polylogarithms", Russian Math. (Iz. VUZ), 38:5 (1994), 47-57.

[103] В.Н. Сорокин, "Аппроксимации Эрмита-Паде для систем Никишина и иррациональность $\zeta(3)$ ", УМH, 49:2(296) (1994), 167-168; англ. пер.: V. N. Sorokin, "Hermite-Padé approximations for Nikishin systems and the irrationality of $\zeta(3)$ ", Russian Math. Surveys, 49:2 (1994), 176-177.

[104] В.Н.Сорокин, "О линейной независимости значений обобщенных полилогарифмов", Матем. сб., 192:8 (2001), 139-154; англ. пер.: V. N. Sorokin, "On linear independence of values of generalized polylogarithms", Sb. Math., 192:8 (2001), 1225-1239.

[105] В. Н. Сорокин, "Циклические графы и теорема Апери", УМН, 57:3(345) (2002), 99-134; англ. пер.: V. N. Sorokin, "Cyclic graphs and Apéry's theorem", Russian Math. Surveys, 57:3 (2002), 535-571.

[106] Дж. Спрингер, Введение в теорию римановых поверхностей, ИЛ, М., 1960, 343 с.; пер. с англ.: G. Springer, Introduction to Riemann surfaces, Addison-Wesley Publishing Company, Inc., Reading, MA, 1957, viii+307 pp.

[107] H. Stahl, "Extremal domains associated with an analytic function. I", Complex Variables Theory Appl., 4 (1985), 311-324.

[108] H. Stahl, "Extremal domains associated with an analytic function. II", Complex Variables Theory Appl., 4 (1985), 325-338.

[109] H. Stahl, "The structure of extremal domains associated with an analytic function", Complex Variables Theory Appl., 4 (1985), 339-354.

[110] H. Stahl, "Orthogonal polynomials with complex-valued weight function. I", Constr. Approx., 2 (1986), 225-240.

[111] H. Stahl, "Orthogonal polynomials with complex valued weight function. II", Constr. approx., 2 (1986), 241-251.

[112] H. Stahl, "Asymptotics of Hermite-Padé polynomials and related convergence results - a summary of results", Nonlinear numerical methods and rational approximation (Wilrijk, 1987), Math. Appl., 43, Reidel, Dordrecht, 1988, 23-53.

[113] H. Stahl, "Conjectures around the Baker-Gammel-Wills conjecture", Constr. Approx., 13:2 (1997), 287-292.

[114] H. Stahl, "The convergence of Padé approximants to functions with branch points", J. Approx. Theory, 91:2 (1997), 139-204.

[115] С. П. Суетин, "Об асимптотике знаменателей диагональных аппроксимаций Паде ортогональных разложений", Докл. РАН, $356: 6$ (1997), 744-746; англ. пер.: S.P. Suetin, "Asymptotics of the denominators of the diagonal Padé approximations of orthogonal expansions", Dokl. Math., 56:2 (1997), 774-776. 
[116] С. П. Суетин, "О равномерной сходимости диагональных аппроксимаций Паде для гиперэллиптических функций”, Матем. сб., 191:9 (2000), 81-114; англ. пер.: S.P. Suetin, "Uniform convergence of Padé diagonal approximants for hyperelliptic functions", Sb. Math., 191:9 (2000), 1339-1373.

[117] С. П. Суетин, "О сходимости чебышёвских непрерывных дробей для эллиптических функций", Матем. сб., 194:12 (2003), 63-92; англ. пер.: S. P. Suetin, "Convergence of Chebyshev continued fractions for elliptic functions", Sb. Math., 194:12 (2003), 1807-1835.

[118] С.П. Суетин, “Численный анализ некоторых характеристик предельного цикла свободного уравнения Ван дер Поля", Совр. пробл. матем., 14, МИАН, М., 2010, 3-57; англ. пер.: S. P. Suetin, "Numerical analysis of some characteristics of the limit cycle of the free van der Pol equation", Proc. Steklov Inst. Math., 278, suppl. 1 (2012), S1-S54.

[119] S. Suetin, On the distribution of zeros of the Hermite-Padé polynomials for three algebraic functions $1, f, f^{2}$ and the global topology of the Stokes lines for some differential equations of the third order, 2013, 59 pp., arXiv: 1312.7105.

[120] Г. Сегё, Ортогональные многочлены, Физматгиз, М., 1962, 500 с.; пер. с англ.: G. Szegö, Orthogonal polynomials, rev. ed., 23, Amer. Math. Soc., Providence, RI, 1959, ix +421 pp.

[121] W. Van Assche, "Padé and Hermite-Padé approximation and orthogonality", Surv. Approx. Theory, 2 (2006), 61-91; 2006, 31 pp., arXiv: math/0609094.

[122] W. Van Assche, "Nearest neighbor recurrence relations for multiple orthogonal polynomials", J. Approx. Theory, 163:10 (2011), 1427-1448.

[123] E. B. van Vleck, "Selected topics in the theory of divergent series and of continued fractions" (Boston colloquium, Sept. 2-5, 1903), Amer. Math. Soc. Coll. Publ., 1, Macmillan, New York, 1905, 75-187.

[124] Р. Варга, Функциональный анализ и теория аппроксимации в численном анализе, Мир, М., 1974, 126 с.; пер. с англ.: R.S. Varga, Functional analysis and approximation theory in numerical analysis, CBMS-NSF Regional Conf. Ser. Appl. Math., 3, SIAM, Philadelphia, PA, 1971, v+76 pp.

[125] P. Vojta, "Diophantine approximation and Nevanlinna theory", Arithmetic geometry, Lecture Notes in Math., 2009, Springer, Berlin, 2011, 111-224.

[126] P. Vojta, "Multiplier ideal sheaves, Nevanlinna theory, and Diophantine approximation", Number theory, analysis and geometry, Springer, New York, 2012, 647-658.

[127] J. T.-Y. Wang, "An effective Schmidt's subspace theorem over function fields", Math. Z., 246:4 (2004), 811-844.

[128] Э. И. Зверович, "Краевые задачи теории аналитических функций в гёльдеровских классах на римановых поверхностях", УМН, 26:1(157) (1971), 113-179; англ. пер.: Ё.I. Zverovich, "Boundary value problems in the theory of analytic functions in Hölder classes on Riemann surfaces", Russian Math. Surveys, 26:1 (1971), 117-192.

\section{Сергей Павлович Суетин (Sergey P. Suetin)}

Российской академии наук

E-mail: suetin@mi.ras.ru 